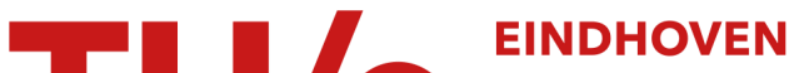 \\ UNIVERSITY OF \\ TECHNOLOGY
}

\section{The computation of properties of injection-moulded products}

Citation for published version (APA):

Douven, L. F. A., Baaijens, F. P. T., \& Meijer, H. E. H. (1995). The computation of properties of injectionmoulded products. Progress in Polymer Science, 20(3), 403-457. https://doi.org/10.1016/0079-6700(94)00037-3

DOI:

10.1016/0079-6700(94)00037-3

Document status and date:

Published: 01/01/1995

Document Version:

Publisher's PDF, also known as Version of Record (includes final page, issue and volume numbers)

Please check the document version of this publication:

- A submitted manuscript is the version of the article upon submission and before peer-review. There can be important differences between the submitted version and the official published version of record. People interested in the research are advised to contact the author for the final version of the publication, or visit the $\mathrm{DOI}$ to the publisher's website.

- The final author version and the galley proof are versions of the publication after peer review.

- The final published version features the final layout of the paper including the volume, issue and page numbers.

Link to publication

\section{General rights}

Copyright and moral rights for the publications made accessible in the public portal are retained by the authors and/or other copyright owners and it is a condition of accessing publications that users recognise and abide by the legal requirements associated with these rights.

- Users may download and print one copy of any publication from the public portal for the purpose of private study or research.

- You may not further distribute the material or use it for any profit-making activity or commercial gain

- You may freely distribute the URL identifying the publication in the public portal.

If the publication is distributed under the terms of Article $25 \mathrm{fa}$ of the Dutch Copyright Act, indicated by the "Taverne" license above, please follow below link for the End User Agreement:

www.tue.nl/taverne

Take down policy

If you believe that this document breaches copyright please contact us at:

openaccess@tue.nl

providing details and we will investigate your claim. 


\title{
THE COMPUTATION OF PROPERTIES OF INJECTION-MOULDED PRODUCTS*
}

\author{
L. F. A. DOUVEN $\uparrow$, F. P. T. BAAIJENS $\uparrow$ and H. E. H. MEIJER $\ddagger$ \\ $\dagger$ Philips Research Laboratories, Prof. Holstlaan 4, 5656 AA Eindhoven, The Netherlands \\ $\$$ Eindhoven University of Technology, Den Dolech 2, $5600 \mathrm{MB}$ Eindhoven, The Netherlunds
}

\begin{abstract}
Injection moulding is a flexible production technique for the manufacture of complex shaped, thin walled polymer products that require minimal finishing. During processing, the polymer experiences a complex deformation and temperature history that affects the final properties of the product.
\end{abstract}

In a growing number of applications, injection-moulded products must meet high demands concerning their properties and dimensional stability. As a consequence, the ultimate aim of numerical simulations of the injection-moulding process is not only to analyse the processing stage but also to calculate the mechanical (and optical) properties of the product, starting from the material properties and the processing conditions. This also requires measurement techniques that can determine molecular orientation, residual stresses and density distributions.

In all recent models of the injection-moulding process, the so-called $2 \frac{1}{2} \mathrm{D}$ approach is employed, referring to limitations of the mould geometry to narrow, weakly curved channels. Thus the ratio of the cavity thickness $h$ and a characteristic length $l$ in the mid-plane of the cavity must be much less than unity. In this paper an attempt is made to model all the stages of the production process, using this $2 \frac{1}{2} \mathrm{D}$ approach. The analysis is restricted to amorphous thermoplastics.

Residual stresses in injection-moulded products stem from two main sources: first, the frozen-in flow-induced stresses, caused by viscoelastic flow of the polymer during the filling and post-filling stage of the injection-moulding process. These stresses correspond with the orientation of macromolecules; second, the thermally- and pressure-induced stresses, which are caused by differential shrinkage. In absolute value, the thermally-induced stresses are usually substantially larger than the frozen-in flowinduced stresses. However, the molecular orientation, as reflected in the frozen-in flow-induced stresses, determines the anisotropy of mechanical, thermal and optical properties and influences the long-term dimensional stability of an injection-moulded product.

A decoupled method is proposed to calculate flow-induced stresses. Firstly, the kinematics of the flow field are determined, employing a viscous, generalized Newtonian constitutive law for the Cauchy stress tensor in combination with the balance laws. This is realized for all stages of the process: injection, packing, holding and cooling. The flow kinematics are subsequently substituted in a viscoelastic constitutive equation to calculate the transient stresses. Two constitutive models are used: a compressible version of the Leonov model (differential formulation) and a compressible version of the Wagner model (integral formulation). In the decoupled method, the flow kinematics are, consequently, supposed not to be influenced by the viscoelastic character of the flowing polymer melt. This decoupled method has a number of advantages compared to a coupled viscoelastic computation: the computation time is reduced considerably, an arbitrary viscoelastic constitutive equation can be employed easily, and no restrictions on the complexity of the flow field are imposed. In the case of $2 \mathrm{D}$ geometries, the validity of this approach is investigated by comparison of the results with those of a fully coupled viscoelastic calculation. These calculations show that the results obtained by the decoupled method are in acceptable agreement with the results of a fully coupled viscoelastic calculation.

For the calculation of thermally-induced stresses a thermo-viscoelastic constitutive law, a linearized form of both viscoelastic constitutive models mentioned above, is employed. In order to attain realistic

*Dedicated to Professor Dr H. Janeschitz-Kriegl on the occasion of his 70th birthday. 
results, special attention must be paid to the boundary conditions. In particular, it is shown that not only the temperature history, but also the pressure history has a marked influence on the residual stress state of an injection-moulded product.

The theories, derived in this paper, are illustrated by a number of examples. Computed results are compared with well documented experimental results from literature. A fair prediction of the properties of injection-moulded products is obtained. It is concluded that for more precise predictions, future attention should be focused on a more accurate and extended determination of the material properties. In particular, non-equilibrium $p \nu T$-data, the pressure dependence of the stationary shear viscosity, and the shear rate dependent first normal stress difference should be measured with great accuracy.

\section{CONTENTS}

Notation

1. Introduction

1.1. Overview of modelling the injection process

1.1.1. Viscous, $1 \frac{1}{2} \mathrm{D}$ approach for the filling stage

1.1.2. Viscous, $2 \frac{1}{2} \mathrm{D}$ approach for the filling stage

1.1.3. Post-filling stages

1.1.4. $1 \frac{1}{2} \mathrm{D}$ approach for flow-induced stresses $\quad 408$

1.1.5. Fountain flow

408

1.1.6. Thermally-induced stresses

408

1.1.7. Dimensions and shape of products $\quad 409$

1.2. Outline

409

2. Constitutive equations

2.1. The Cauchy stress tensor

2.1.1. Scope of this section

2.1.2. The compressible Leonov model

2.1.3. The compressible Wagner model

2.1.4. Generalized Newtonian fluid model

2.1.5. Thermo-rheologically simple materials

2.1.6. Linear thermo-viscoelasticity

2.1.7. The $p \nu T$-relation $\quad 415$

2.2. Thermal properties 416

2.3. Summary 416

3. Modelling the injection-moulding process 416

3.1. Introduction 416

3.2. Analysis of compressible, solidifying, generalized Newtonian flow 417

$\begin{array}{ll}\text { 3.2.1. Thin film approximation } & 417\end{array}$

3.2.2. Pressure problem 420

3.2.3. Temperature problem 421

3.3. Flow-induced stresses 422

3.3.1. Introduction 422

3.3.2. Decoupled method: the compressible Leonov model 423

3.3.3. Decoupled method: the compressible Wagner model 423

3.4. Thermally- and pressure-induced stresses 424

3.4.1. Introduction 424

3.4.2. Linear thermo-viscoelastic modelling of thermal stresses 424

3.4.3. Numerical solution of the thermal stress problem 425

3.5. Conclusion 428 
4. Material characterization $\quad 429$

$\begin{array}{lr}\text { 4.1. Introduction } & 429\end{array}$

$\begin{array}{lr}\text { 4.2. Rheological characterization } & 429\end{array}$

4.2.1. Linear viscoelastic measurements in the melt 429

4.2.2. The damping function $\quad 431$

4.2.3. Comparison of the Leonov and Wagner model 432

4.2.4. Steady state shear viscosity $\quad 434$

4.2.5. Linear viscoelastic measurements in the glass 435

4.3. $p \nu T$-data and thermal properties $\quad 438$

4.3.1. $p \nu T$-data $\quad 438$

$\begin{array}{ll}\text { 4.3.2. Thermal conductivity, } \lambda & 438\end{array}$

4.3.3. Heat capacity at constant pressure, $c_{p} \quad 440$

5. Numerical simulations $\quad 440$

$\begin{array}{lr}\text { 5.1. Introduction } & 440\end{array}$

$\begin{array}{lr}\text { 5.2. Flow-induced stresses } & 441\end{array}$

5.2.1. Mould geometry $\quad 441$

5.2.2. Processing conditions $\quad 442$

5.2.3. Results $\quad 442$

5.3. Thermally-induced stresses $\quad 446$

$\begin{array}{ll}\text { 5.3.1. Results } & 446\end{array}$

$\begin{array}{ll}\text { 5.3.2. Case no. } 1 & 447\end{array}$

5.3.3. Case no. 2

6. Conclusions

References $\quad 454$

\section{NOTATION}

\section{Quantities}

\begin{tabular}{|c|c|}
\hline $\begin{array}{l}A, a \\
\overrightarrow{\mathrm{a}} \\
\mathbf{A},{ }^{4} \mathbf{A} \\
\underset{\tilde{A}}{A} \\
\stackrel{\mathbf{I}}{\mathbf{I}}^{4}{ }^{4} \mathbf{I}\end{array}$ & $\begin{array}{l}\text { scalar } \\
\text { vector } \\
\text { second, fourth order tensor } \\
\text { column } \\
\text { matrix } \\
\text { second, fourth order unit tensor }\end{array}$ \\
\hline
\end{tabular}

Operations and Functions

$\mathbf{A}^{c},{ }^{4} \mathbf{A}^{c}$

$\vec{a} \vec{b}, \mathbf{A} \vec{b}, \vec{a} \mathbf{B}, \mathbf{A B}$

conjugation

$\vec{a} \cdot \vec{b}, \mathbf{A} \cdot \vec{b}, \vec{a} \cdot \mathbf{B}, \mathbf{A} \cdot \mathbf{B}$ inner product

$\mathbf{A}: \mathbf{B},{ }^{4} \mathbf{A}: \mathbf{B}$

$\mathbf{A}^{-1}, \underline{A}^{-1}$

double inner product

$\|\vec{a}\|,\|\mathbf{A}\|$

inversion

$I_{1}^{\mathbf{A}} \equiv \operatorname{tr}(\mathbf{A})$

norm

$I_{2}^{\mathrm{A}}$

first invariant of a second order tensor, or trace

second invariant of a second order tensor

$I_{3}^{\mathbf{A}} \equiv \operatorname{det}(\mathbf{A})$

$\mathbf{A}^{d}=\mathbf{A}-\frac{1}{3} \operatorname{tr}(\mathbf{A}) \mathbf{I}$

third invariant of a second order tensor, or determinant deviatoric part of a second order tensor 


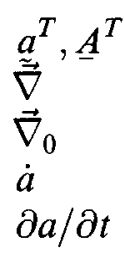

transposition

gradient operator

gradient operator with respect to the reference configuration material time derivative

spatial time derivative

\section{INTRODUCTION}

As injection-moulding is a complex process, a number of problems may arise that affect product quality. A good understanding of the process will help the product and mould designer to circumvent these difficulties. Some of these issues are listed below.

- The filling pattern of the mould is crucial for the evaluation of a number of details in the mould design:

- location of the vents, necessary to release air entrapped in the mould.

- location and occurrence of weld lines. This is the area in which two flow fronts meet as the cavity is filled; weld lines are weak.

- location of the gates.

- Balancing the runner system.

- The location of the cooling channels has a great effect on the cycle time, on the temperature field of the cavity surface, and consequently on the thermal stresses and the warpage.

- Viscous heating may cause, locally, an excessive temperature rise, resulting in material degradation.

- Shrinkage occurs when insufficient material is injected into the mould.

- Over-packing occurs when too much material is forced into the mould, resulting in difficulties for ejecting the product from the mould.

- Molecular orientation is partly frozen-in, as relaxation is prevented by rapid cooling.

- The injection-moulded product possesses residual stresses, mainly due to differential shrinkage.

- Warpage occurs when the shrinkage is different over the product. This may be caused by inhomogeneous cooling or by frozen-in molecular orientation.

- Aging of the injection-moulded product causes instability of the dimensions of the product during its life.

Many researchers have focused their attention on the injection-moulding process, aiming to solve the above mentioned problems and optimization of the process. The next sections deal with a number of aspects that have been investigated.

\subsection{Overview of Modelling the Injection Process}

\subsubsection{Viscous, $1 \frac{1}{2} D$ Approach for the Filling Stage}

Effort has been directed to the analysis of the filling stage, aimed at the prediction of the filling pattern and the pressure and/or temperature distribution. At first, only 
uni-directional flow between two parallel plates, separated by a narrow gap, was considered. Geometries were either circular or rectangular. The temperature field was two-dimensional, depending on the flow coordinate and the perpendicular coordinate along the thickness direction. Generalized Newtonian fluid behaviour was adopted, where the temperature dependence of the viscosity is responsible for the coupling of the balance equations. This is called the $1 \frac{1}{2} \mathrm{D}$ approach. The equations were solved with analytical and numerical techniques (finite difference method). Contributions in this field worth mentioning are: Pearson, ${ }^{77}$ Chaptcr 4; Harry and Parrot $^{31}$; and Wu et al. ${ }^{110}$ Numerical methods have become more sophisticated. A good example of this development can be found in the papers of Williams and Lord, ${ }^{107}$ and Lord and Williams, ${ }^{64}$ who in addition modelled the flow in circular runners.

Broyer et al. ${ }^{10}$ analyzed two-dimensional isothermal Newtonian flow. In a subsequent paper Tadmor et al. ${ }^{93}$ extended this approach to non-Newtonian melt behaviour.

\subsubsection{Viscous, $2 \frac{1}{2} D$ Approach for the Filling Stage}

A development towards more realistic geometries was one of the next steps. Richardson et al. ${ }^{80}$ proposed to combine a number of basic $1 \frac{1}{2} \mathrm{D}$ flow geometries to form a complex shaped cavity. These basic flow geometries were, e.g. a rectangular channel, a circular pipe, and a disk. A more general approach, based on combined finite element and finite difference analysis was first introduced by Hieber and Shen ${ }^{34}$ and Hieber $e t a l^{35}$ This approach was later followed and improved by many others, including Sitters ${ }^{87}$ and Boshouwers and van der Werf. ${ }^{8}$ This method is called the $2 \frac{1}{2} \mathrm{D}$ approach, because the pressure field is solved in two dimensions by means of the finite element method, and the temperature and velocity field in three dimensions by means of the finite difference method. This method is currently applied in nearly all up to date commercial software available in this field. The finite element method is capable of handling geometries of arbitrary shape. The finite difference scheme accounts for an accurate description of large gradients in the thickness direction, without the need for excessive computing times. An alternative method, utilizing a numerically generated finite difference grid of complex shape, was proposed by Subbiah et al..$^{91}$ This method requires a new grid for every time step, and is restricted to planar geometries.

\subsubsection{Post-Filling Stages}

The analysis of the transient flow and temperature fields, during the post-filling stages of the process, requires an account of the compressibility of the polymer material. The papers by Kamal and $\mathrm{Kenig}^{46,47}$ and Kamal et al. ${ }^{48}$ form an early attempt to extend the $1 \frac{1}{2} \mathrm{D}$ approach to the packing and holding stagc of the process. More recently, Kamal and Lafleur ${ }^{49,50}$ and Lafleur and Kamal ${ }^{52}$ analyzed the entire injection-moulding cycle, taking into account viscoelastic material behaviour. Other papers in this field were by Greener, ${ }^{29,30}$ who investigated the influence of the pressure and temperature history on the density distribution of an injection-moulded sample. However, no flow analysis was taken into account and the approach is 
analytical, involving a number of simplifying assumptions. Chung et al. ${ }^{16-18}$ gave an analysis of the pressure field during the short packing stage in a rectangular cavity, assuming Newtonian fluid behaviour and a constant temperature. A concise $1 \frac{1}{2} \mathrm{D}$ analysis of the whole injection-moulding cycle was given by Hieber, ${ }^{33}$ Section $1 . I V$, and recently by Chiang. ${ }^{14,15}$

\subsection{4. $1 \frac{1}{2} D$ Approach for Flow-Induced Stresses}

In order to calculate flow-induced stresses, application of more realistic material models was necessary. Isayev and Hieber ${ }^{42}$ and Isayev ${ }^{40}$ applied the Leonov constitutive equation to simulate the injection and cooling stage of the injection-moulding process, predicting birefringence in injection-moulded polystyrene samples. Wales ${ }^{104}$ presented birefringence measurements and related these to the shear stresses at the wall during filling, later followed by Tadmor ${ }^{92}$ and Kamal and Tan. ${ }^{51}$ JaneschitzKriegl $^{44,45}$ investigated the results of Wales by means of an analytical model for the heat transfer in the injection-moulded sample during filling. Another approximate theory was proposed by Dietz and White, ${ }^{20}$ Dietz et al., ${ }^{21}$ and White and Dietz. ${ }^{105}$ They applied a viscous model in the injection stage, calculating normal stresses from shear stresses by means of an empirical relation. After cessation of flow, relaxation of the stresses was calculated by means of a viscoelastic model (integral Maxwell model). More recently, Kiou and Suh, ${ }^{62}$ following Isayev and Hieber, ${ }^{42}$ showed the effect of an insulation layer applied on the cavity surface on the reduction of frozen-in birefringence. Baaijens and Douven ${ }^{3}$ showed the development of flow-induced stresses during the entire production cycle, including the packing and holding stage, applying a compressible version of the Leonov model. Flaman ${ }^{26}$ applied this model in predicting frozen-in birefringence in an injection-moulded strip and showed that the calculated results compared well with measurements. All these papers concentrated on the $1 \frac{1}{2} \mathrm{D}$ approach for a rectangular cavity.

\subsubsection{Fountain Flow}

Several authors addressed the influence of the fountain flow, the extensional flow occurring just behind the advancing melt front, on product properties (see e.g. Tadmor, ${ }^{92}$ Dietz and White ${ }^{20}$ ). Bhattacharji and Savic ${ }^{6}$ derived an analytical expression for the flow field in a flat fountain flow region. Mavridis et al. ${ }^{68}$ gave numerical results of the fountain flow.

\subsubsection{Thermally-Induced Stresses}

Thermally-induced stresses that develop in an injection-moulded product during processing can be modelled with linear thermo-viscoelastic material behaviour (Muki and Sternberg; ${ }^{70}$ Lee and Rogers ${ }^{56}$ ). These theories were first successfully applied to the free quenching of glass sheets (Lee et al. ${ }^{57}$ Narayanaswamy and Gardon; $;^{72}$ Ohlberg and Woo; ${ }^{75}$ and Gardon ${ }^{28}$ ). Free quenching of polymers was analyzed, numerically and experimentally by Struik, ${ }^{90}$ Wimberger et al., ${ }^{109}$ Frutiger 
and Woo, ${ }^{27}$ Saffell and Windle, ${ }^{82}$ Wust and Bogue, ${ }^{111}$ Maneschy et al. ${ }^{67}$ and Lee et $a l .{ }^{58}$ The characteristic result is a parabolic stress distribution, displaying compressive stresses at the surface balanced by tensile stresses in the centre region. However, the residual thermal stress distribution in an injection-moulded sheet differs from the one caused by free quenching, because the melt pressure fixes the frozen layers at the cavity surfaces. Tensile stresses were reported at the surface of an injection-moulded product (Titomanlio et al. ${ }^{95}$ Rezayat; ${ }^{78}$ Baaijens $^{2}$ and Smits ${ }^{88}$ ). These calculated results were confirmed by measurements of Zoetelief ${ }^{12}$ and Schennink. ${ }^{83}$ However, measured parabolic-like stress profiles were reported as well for injection-moulded plates (Wagner et al..$^{99}$ Thompson and White ${ }^{94}$ ).

Several studies illustrated the influence of residual stresses on mechanical performance of quenched polymers and injection-moulded products. Attention is often focused on the failure of polymer materials. Broutman and Krishnakumar ${ }^{9}$ and So and Broutman ${ }^{89}$ investigated the influence of residual stresses on impact strength. White et al. ${ }^{106}$ treated the crazing and environmental stress cracking in polymer materials. Fatigue behaviour of injection-moulded samples was studied by Mandell et al.$^{66}$ and by Iacopi and White. ${ }^{39}$ Siegmann et al ${ }^{85}$ investigated the effect of injectionmoulding process conditions on mechanical behaviour of the product in a rather general way. Obviously, the prediction of residual stresses, in injection-moulded products of an arbitrary shape, is an important objective for the modelling of the total process.

\subsubsection{Dimensions and Shape of Products}

For precision injection-moulding, an accurate prediction of the evolution of the dimensions and shape of the product is required. This involves prediction of shrinkage (Isaye ${ }^{41}$ ) and post-shrinkage. In a simplified analysis, Jacques ${ }^{43}$ focused on the calculation of warpage due to residual stresses. Post-shrinkage is governed by a slow relaxation of frozen-in orientation and volume relaxation, caused by the nonequilibrium density distribution. Obviously, the frozen-in orientation, residual stress state and aging behaviour of the material must be established in order to model precision injection-moulding. Clearly this has not yet been accomplished for $2 \frac{1}{2} \mathrm{D}$ geometries.

\subsection{Outline}

The objective of this paper is to analyze the entire injection-moulding process, modelling not only flow, pressure, and temperature distributions, but also the frozen-in orientation, the residual stress state and the transient dimensions and shape of a $2 \frac{1}{2} \mathrm{D}$, amorphous thermoplastic product.

In Section 2 of this paper, a brief introduction is given to continuum mechanics and thermodynamics. The aim is directed at the determination of fields of density, motion and temperature of a body. These fields must first of all obey the balance laws, a set of coupled non-linear differential equations that contain an additional number of dependent variables (in contrast to the above mentioned fields of density, motion 
and temperature, which are called independent variables). The dependent variables (the Cauchy stress tensor, the internal energy, the heat flux vector, and the entropy) represent specific material behaviour and are discussed in this section.

In Section 3 the theoretical framework of Section 2 is applied to the injectionmoulding of thermoplastics in narrow, weakly curved channels, leading to a so-called $2 \frac{1}{2} \mathrm{D}$ model. All consecutive stages of the production cycle are modelled. A number of aspects of the process, like frozen-in orientation and residual stresses, are highlighted.

In Section 4 the material data, required as input for the calculations, are presented. Two materials are characterized: a polystyrene and a polycarbonate.

In Section 5 results of some calculations are given, illustrating the capabilities of the numerical tools developed. Finally, in Section 6 the main results of the present research are summarized and suggestions for further research are given.

\section{CONSTITUTIVE EQUATIONS}

Continuum mechanics is concerned with the thermo-mechanical behaviour of continuous media on a macroscopic scale. The main goal of continuum mechanics is to determine the fields of density, temperature and motion for all material points considered, as a function of time (Müller, ${ }^{71}$ Section 1.1). Constitutive equations are needed to solve the equations of balance. The balance of mass, momentum, moment of momentum, and energy are given by, respectively,

$$
\begin{gathered}
\dot{\rho}+\rho \vec{\nabla} \cdot \overrightarrow{\mathrm{v}}=\dot{\rho}+\rho \operatorname{tr}(\mathbf{D})=0 \\
\vec{\nabla} \cdot \boldsymbol{\sigma}^{c}+\rho \overrightarrow{\mathrm{f}}=\rho \dot{\overrightarrow{\mathrm{v}}} \\
\boldsymbol{\sigma}=\boldsymbol{\sigma}^{c} \\
\rho \dot{e}=\boldsymbol{\sigma}: \mathbf{D}-\vec{\nabla} \cdot \overrightarrow{\mathrm{h}}+\rho r
\end{gathered}
$$

where $\rho$ denotes the density, $\mathbf{D}$ is the deformation rate tensor, $\boldsymbol{\sigma}$ is the Cauchy stress tensor, $\overrightarrow{\mathrm{f}}$ is the specific (i.e. per unit mass) body force, $e$ is the specific internal energy, $\overrightarrow{\mathrm{h}}$ is the heat flux vector and $r$ is the specific heat source. It is assumed that the specific body force $\overrightarrow{\mathrm{f}}$ and the specific heat source $r$ are known functions of position and time. Three additional fields $-\sigma, \overrightarrow{\mathrm{h}}$ and $e-$ are introduced in the balance laws. This implies that constitutive relations, expressing these fields as functions of density, temperature and motion, must be established. These relations represent specific material behaviour. $\sigma, \overrightarrow{\mathrm{h}}$ and $e$ are called the dependent variables and $\rho, T$ and position $\vec{x}$ are called the independent variables. In Sections $2.1-2.2$ the constitutive equations that will be employed in this paper are discussed.

\subsection{The Cauchy Stress Tensor}

\subsubsection{Scope of this Section}

A description is given of all the constitutive equations employed for the Cauchy stress tensor to model the injection-moulding process in this paper. 
The compressible Leonov model, a viscoelastic differential constitutive equation (Section 2.1.2), and the compressible Wagner model, a viscoelastic integral constitutive equation (Section 2.1.3), are both used to calculate flow-induced residual stresses. The generalized Newtonian fluid model presented in Section 2.1.4 neglects all elastic effects and is used to determine the flow kinematics.

Section 2.1.5 deals with thermo-rheological simple materials. The linear thermoviscoelastic model, discussed in Section 2.1.6, will be applied to model the behaviour of the solidified material during and after moulding. Displacement gradients are assumed to be small and the model can be obtained by a linearization of the Leonov model, or the Wagner model, or it can be derived from a general expression for $\sigma$. Thus the constitutive behaviour in both the melt and the solid state is modelled by a consistent set of constitutive equations.

In Section 2.1.7, a constitutive relation for the hydrostatic part of the Cauchy stress tensor is given in an implicit form, the so-called $p \nu T$-relation. This model applies for all constitutive models derived in this section.

\subsubsection{The Compressible Leonov Model}

An outline is given of the fundamentals of the compressible Leonov model. For further details the reader is referred to Leonov ${ }^{59,60}$ and Baaijens. ${ }^{1}$

Let $\mathbf{F}$ be the deformation tensor, defined as $\mathbf{F}=\left(\vec{\nabla}_{0} \overrightarrow{\mathbf{x}}\right)^{c}$. Following Simo, ${ }^{86}$ volumetric effects in $\mathbf{F}_{e}$, the elastic part of the deformation tensor, are separated from the deviatoric effects by defining

$$
\overline{\mathbf{F}}_{e}=J^{-1 / 3} \mathbf{F}_{e}, \quad \bar{J}_{e}=\operatorname{det}\left(\overline{\mathbf{F}}_{e}\right)=1
$$

The Finger strain tensor associated with $\overline{\mathbf{F}}_{e}$ is defined as

$$
\overline{\mathbf{B}}_{e}=\overline{\mathbf{F}}_{e} \cdot \overline{\mathbf{F}}_{e}^{c}
$$

The predictive quality of the Leonov constitutive equation is enhanced by considering a multi-mode model containing a number $(m)$ of viscosities $\eta_{i}$ and relaxation times $\theta_{i}$. For the multi-mode case the compressible Leonov model can be summarized as

$$
\begin{aligned}
& \boldsymbol{\sigma}=-\rho \mathbf{I}+\boldsymbol{\sigma}_{e}^{d}+\boldsymbol{\sigma}_{p} \\
& \boldsymbol{\sigma}_{e}^{d}=\sum_{i=1}^{m} \frac{\eta_{i}}{\hat{\theta}_{i}} \overline{\mathbf{B}}_{e i}^{d}, \quad \sigma_{p}=2 \eta_{r} \mathbf{D}^{d} \\
& \nu=\nu(p, T) \\
& \mathbf{D}_{p i}=\frac{1}{4 \theta_{i}}\left(\overline{\mathbf{B}}_{e i}^{d}-\overline{\mathbf{B}}_{e i}^{-d}\right) \\
& \dot{\overline{\mathbf{B}}}_{e i}=\left(\begin{array}{ll}
\mathbf{L}^{d} & \mathbf{D}_{p i}
\end{array}\right) \cdot \overline{\mathbf{B}}_{e i}+\overline{\mathbf{B}}_{e i} \cdot\left(\mathbf{L}^{d c}-\mathbf{D}_{p i}\right)
\end{aligned}
$$

where $p$ is the hydrostatic pressure, $\nu$ is the specific volume, $\sigma_{e}$ and $\sigma_{p}$ are the elastic and plastic part of the Cauchy stress tensor. $\mathbf{L}=(\vec{\nabla} \overrightarrow{\mathrm{v}})^{c}$ denotes the velocity gradient, 
$\mathbf{D}=1 / 2\left(\mathbf{L}+\mathbf{L}^{c}\right)$ the deformation rate tensor and $\mathbf{D}_{p i}$ the plastic part of the deformation rate tensor.

For small strains the model can be linearized. The parameters $\eta_{i}, \theta_{i}$ and $\eta_{r}$ can be determined by measuring the linear viscoelastic material functions only (Leonov ${ }^{61}$ ).

The temperature dependency of $\theta_{i}, \eta_{i}$ and $\eta_{r}$ is treated in Section 2.1.5.

\subsubsection{The Compressible Wagner Model}

The Wagner model (Wagner ${ }^{100,101}$ ) can be regarded as a special case of the $K-B K Z$ model. The compressible version of the Wagner model is derived in Douven ${ }^{22}$ and reads

$$
\boldsymbol{\sigma}=-p(\rho(t)) \mathbf{I}+\int_{-\infty}^{t} \boldsymbol{M}(t-\tau) h\left(\overline{\mathscr{I}}_{1}, \overline{\mathscr{I}}_{2}\right) \overline{\mathbf{C}}_{t}^{-d}(\tau) d \tau, \quad h=\frac{\partial H}{\partial \overline{\mathscr{I}}_{2}}
$$

The deformation measure used in this equation is defined as

$$
\overline{\mathbf{C}}_{t}=\overline{\mathbf{F}}_{t}^{c} \cdot \overline{\mathbf{F}}_{t}
$$

where the suffix $t$ signifies that the configuration at time $t$ is taken as the reference configuration. A kinematic split of the deformation tensor $\mathbf{F}_{t}$ separates the deviatoric effects $\overline{\mathbf{F}}_{t}$ and the volumetric effects $\left(J_{t}^{1 / 3}\right)$ :

$$
\mathbf{F}_{t}=J_{t}^{1 / 3} \overline{\mathbf{F}}_{t}, \quad \operatorname{det}\left(\overline{\mathbf{F}}_{t}\right)=1, \quad \operatorname{det}\left(\mathbf{F}_{t}\right)=J_{t}
$$

The memory function $M$ can be obtained by measurements of linear viscoelastic material functions only. The function $h$, called the damping function, must be obtained by measurements in the non-linear regime. The name damping function is explained by the fact that the function is unity in the linear viscoelastic case, where $\mathscr{I}_{1} \approx 3$ and $\mathscr{I}_{2} \approx 3$, and decays toward zero for increasing $\mathscr{I}_{1}$ and $\mathscr{I}_{2}$ (Wagner ${ }^{102}$ ). So $h$ must obey $h(3,3)-1$. Various forms for $h$ have been proposed. Wagner et al. ${ }^{103}$ chosc

$$
\begin{gathered}
h\left(\overline{\mathscr{I}}_{1}, \overline{\mathscr{I}}_{2}\right)=m^{*} \exp \left(-n_{1} \sqrt{I-3}\right)+\left(1-m^{*}\right) \exp \left(-n_{2} \sqrt{I-3}\right) \\
I=\alpha \overline{\mathscr{I}}_{1}+(1-\alpha) \overline{\mathscr{I}}_{2}
\end{gathered}
$$

whereas Papanastasiou et al. ${ }^{76}$ proposed

$$
h\left(\overline{\mathscr{I}}_{1}, \overline{\mathscr{I}}_{2}\right)=\frac{1}{1+\beta(I-3)}
$$

where $I$ is given by eq. $(15)_{2}$.

In non-isothermal situations the memory function $M$ and the pressure $p$ will also depend on the temperature $T$ :

$$
M=M(t-\tau, T) ; \quad p=p(\rho, T)
$$

A possible constitutive equation for $p$ is given by the Tait equation; (see Section 2.1.7).

2.1.3.1. Specification of the Memory Function $-M$ is chosen in a form that is able to fit experimental data accurately and that is convenient for numerical evaluation of the 
stresses in instationary flows (Ferry, ${ }^{25}$ Chapter 3 ):

$$
M(t-\tau, T)=\int_{-\infty}^{\infty} \frac{H(\theta)}{\theta} e^{-(t-\tau) / \theta} \mathrm{d} \ln \theta
$$

where $H(\theta)$ is a continuous relaxation spectrum and $\theta=\theta(T)$. If $H$ is chosen as a discrete spectrum obeying

$$
H(\theta)=\sum_{i=1}^{m} G_{i} \theta_{i} \delta\left(\theta-\theta_{i}\right)
$$

where $\delta$ is the delta function, the memory function is identical to the multi-mode Maxwell medium:

$$
M(t-\tau, T)=\sum_{i=1}^{m} \frac{G_{i}}{\theta_{i}} e^{-(t-\tau) / \theta_{i}}
$$

The temperature dependency of $M$ is treated in Section 2.1.5.

\subsubsection{Generalized Newtonian Fluid Model}

Ignoring elastic effects and assuming generalized Newtonian behaviour, i.e. $\sigma^{d}$ is linear in $\mathbf{D}^{d}$, the material behaviour of the polymer melt can be represented by a compressible, heat conducting, generalized Newtonian fluid model. Application of the Clausius-Duhem inequality results in the following expression for the Cauchy stress tensor (Sitters, ${ }^{87}$ Chapter 2):

$$
\begin{gathered}
\boldsymbol{\sigma}=-p \mathbf{I}+\boldsymbol{\sigma}^{d} \\
p=p_{0}-\mu \operatorname{tr}(\mathbf{D}), \quad \boldsymbol{\sigma}^{d}=2 \eta \mathbf{D}^{d} \\
\mu=\mu\left(p_{0}, T, \dot{\rho}, I_{2}^{\mathbf{D}^{d}}, I_{3}^{\mathbf{D}^{d}}\right), \quad \eta=\eta\left(p_{0}, T, \dot{\rho}, I_{2}^{\mathrm{D}^{d}}, I_{3}^{\mathbf{D}^{d}}\right)
\end{gathered}
$$

where $\mu$ and $\eta$ are the bulk and shear viscosity respectively, $p$ is the hydrostatic pressure. Both $\mu$ and $\eta$ must be greater than or equal to zero.

The hydrostatic pressure $p$ is split into two parts. The first part is the so-called thermodynamic pressure $p_{0}$ representing resistance against static volume changes, the second part is the term $-\mu t r(\mathbf{D})$ representing the viscous part of $p$.

By employing the constitutive principles, the following set of independent variables emerges: $p_{0}, \dot{\rho}, T, I_{2}\left(\mathbf{D}^{d}\right), I_{3}\left(\mathbf{D}^{d}\right)$. Note that $p_{0}$ is chosen as an independent variable instead of $\rho$, and $I_{1}(\mathbf{D})$ is substituted by $\dot{\rho}$ by use of the continuity equation (1). Because $\rho$ is chosen as a constitutive field, a constitutive relation must be given

$$
\rho=\rho\left(p_{0}, T\right)
$$

This relation is specified in Section 2.1.7.

2.1.4.1. Steady State Shear Viscosity - In Section 3 it is shown that shear flow (e.g. $\mathbf{D}^{d}=\dot{\gamma} / 2\left(\overrightarrow{\mathrm{e}}_{1} \overrightarrow{\mathrm{e}}_{3}+\overrightarrow{\mathrm{e}}_{3} \overrightarrow{\mathrm{e}}_{1}\right)$, where $\dot{\gamma}$ is the shear rate) suffices for the modelling of the injection-moulding of thin walled products. The steady state shear viscosity for simple 
shear flow, in case of the Leonov and the Wagner model reads, respectively:

$$
\begin{gathered}
\eta=\eta_{r}+\sum_{i=1}^{m} \frac{2 \eta_{i}}{1+X_{i}}, \quad X_{i}=\sqrt{1+\left(2 \theta_{i} \dot{\gamma}\right)^{2}} \\
\eta=\sum_{i=1}^{m}\left[m^{*} \frac{\eta_{i}}{\left(1+n_{1} \theta_{i} \dot{\gamma}\right)^{2}}+\left(1-m^{*}\right) \frac{\eta_{i}}{\left(1+n_{2} \theta_{i} \dot{\gamma}\right)^{2}}\right]
\end{gathered}
$$

For the Wagner model, the damping function according to eq. (15) is employed. Another model, not derived from a viscoelastic constitutive law, is the Cross model used by Hieber, ${ }^{33}$ Section 1.II.

$$
\eta\left(\dot{\gamma}, T, p_{0}\right)=\frac{\eta_{0}\left(T, p_{0}\right)}{1+\left(\eta_{0} \dot{\gamma} / \tau^{*}\right)^{(1-n)}}
$$

where $\tau^{*}$ and $n$ are constants and $\eta_{0}$ is the zero shear rate viscosity. The temperature and pressure dependency of $\eta$ is treated in the next section.

\subsubsection{Thermo-Rheologically Simple Materials}

For a set of isothermal creep or relaxation curves measured at different temperatures, Leaderman ${ }^{55}$ found that these curves can be shifted onto a master curve by a shift along the logarithmic time axis. The shift depends on the temperature difference between the master curve and the curve that is shifted. This behaviour is commonly observed for many polymers. Materials that obey this rule are called thermorheologically simple. Also a small vertical shift of the curves, due to thermal expansion and influence of temperature, is observed (see Ferry, ${ }^{25}$ Chapter 11). The decrease in relaxation time and viscosity, with increasing temperature, is assumed to behave according to

$$
\begin{gathered}
\theta(T)=a\left(T, T_{0}\right) \theta\left(T_{0}\right)=a_{T} \theta_{0} \\
\eta(T)=a\left(T, T_{0}\right) \frac{\rho T}{\rho\left(T_{0}\right) T_{0}} \eta\left(T_{0}\right)=a_{T} b_{T} \eta_{0}
\end{gathered}
$$

where $T_{0}$ is the reference temperature corresponding to the temperature of the master curve. The term $(\rho T) /\left(\rho_{0} T_{0}\right)$, that is responsible for the vertical shift of isothermal creep or relaxation curves, is close to unity and therefore often omitted. The so-called time-temperature shift function $a_{T}$ is a material property, obeying

$$
a\left(T_{0}, T_{0}\right)=1 ; \quad a_{T}>0 \quad \forall_{T>0} ; \quad \frac{d a_{T}}{d T}<0 \quad \forall_{T>0}
$$

Williams et al. ${ }^{108}$ found that for amorphous polymers in the range of the glass transition temperature $T_{g}$ to about $T_{g}+100 \mathrm{~K}$, the shift function, for shear behaviour, is governed by

$$
\log a_{T}=\frac{c_{1}\left(T-T_{0}\right)}{c_{2}+T-T_{0}}
$$


where $c_{1}$ and $c_{2}$ are more or less universal constants. Pressure dependence of the shift factor was incorporated by Hieber, ${ }^{33}$ Section 1.II, in the following way

$$
T_{0}(p)=T_{0}(0)+s p, \quad c_{2}(p)=c_{2}(0)+s p,
$$

where $s$ is a constant.

\subsubsection{Linear Thermo-Viscoelasticity}

Assuming that time-temperature superposition applies, the linear thermo-viscoelastic constitutive equation is given by

$$
\begin{gathered}
\boldsymbol{\sigma}^{d}=\sum_{i=1}^{m} 2 \int_{0}^{t} G_{i} e^{-(\xi(t)-\xi(\tau)) / \theta_{i 0}} \dot{\epsilon}^{d} \mathrm{~d} \tau, \quad G_{i}=\frac{\eta_{i 0}}{\theta_{i 0}}, \\
p^{h}=\int_{0}^{t}\left(\frac{\alpha}{\kappa} \dot{T}-\frac{1}{\kappa} \operatorname{tr}(\dot{\boldsymbol{\epsilon}})\right) \mathrm{d} \tau,
\end{gathered}
$$

where the so-called reduced time $\xi$ is defined as

$$
\xi(\tau)=\int_{0}^{\tau} \frac{1}{a_{T}} \mathrm{~d} s,
$$

and where $\alpha$, the volume expansion coefficient and $\kappa$, the isothermal compressibility coefficient, are defined as

$$
\alpha=\frac{1}{\nu}\left(\frac{\partial \nu}{\partial T}\right)_{p}, \quad \kappa=-\frac{1}{\nu}\left(\frac{\partial \nu}{\partial p}\right)_{T}
$$

\subsubsection{The p $\nu$ T-Relation}

A constitutive relation for the hydrostatic part of the Cauchy stress tensor is given in an implicit form, the so-called $p \nu T$-relation. An example of this relation is the socalled Tait equation for amorphous polymers $\left(\right.$ Zoller $\left.^{113}\right)$, given by

$$
\begin{aligned}
& \nu(p, T)= \begin{cases}\left(a_{0 m}+a_{1 m}\left(T-T_{g}\right)\right)\left(1-0.0894 \ln \left(1+\frac{p}{B_{m}}\right)\right), & \text { if } T \geq T_{g} ; \\
\left(a_{0 s}+a_{1 s}\left(T-T_{g}\right)\right)\left(1-0.0894 \ln \left(1+\frac{p}{B_{s}}\right)\right), & \text { if } T \leq T_{g}\end{cases} \\
& B_{m}(T)=B_{0 m} e^{\left(-B_{1 m} T\right)}, \quad B_{s}(T)=B_{0 s} e^{\left(-B_{1 s} T\right)} \\
& T_{g}(p)=T_{g}(0)+s p
\end{aligned}
$$

where $T_{g}$ is the pressure-dependent glass transition temperature and $a_{0 m}, a_{1 m}, B_{0 m}$, $B_{1 m}, a_{0 s}, a_{1 s}, B_{0 s}, B_{1 s}$ and $s$ are constants. 


\subsection{Thermal Properties}

The heat flux vector $\overrightarrow{\mathrm{h}}$ is assumed to be proportional to the temperature gradient

$$
\overrightarrow{\mathrm{h}}=-\lambda \cdot \vec{\nabla} T
$$

This constitutive equation is known as Fourier's law; $\lambda$ is called the thermal conductivity tensor. It must be semi-positive definite and depends on the independent variables. In this survey only isotropic heat conduction is taken into account.

Ignoring elastic effects, the specific internal energy $e$ can be written as (see Bird et al., ${ }^{7}$ Chapter 10; Sitters, ${ }^{87}$ Chapter 2):

$$
\begin{gathered}
\dot{e}=c_{p} \dot{T}+\frac{p_{0}}{\rho^{2}} \dot{\rho}+\frac{T}{\rho^{2}}\left(\frac{\partial \rho}{\partial T}\right)_{p_{0}} \dot{p}_{0} \\
c_{p}=c_{p}\left(p_{0}, T\right)=\left(\frac{\partial g}{\partial T}\right)_{p_{0}}
\end{gathered}
$$

where $c_{p}$ is the heat capacity at constant thermodynamic pressure and $g=e+p_{0} / \rho$ is the specific enthalpy. Equation (2.41) is a convenient relation for the internal energy because it is expressed in terms of measurable quantities.

Note that these constitutive equations for the thermal properties will be employed for viscous and viscoelastic material behaviour, although for viscoelastic media eq. (2.41) is only allowed if the contribution of the elastic stresses to the mechanical dissipation is negligible.

\subsection{Summary}

A summary of the main results obtained in Section 2.1 is given in Table 1.

\section{MODELLING THE INJECTION-MOULDING PROCESS}

\subsection{Introduction}

In this chapter the balance laws and constitutive equations, discussed in Section 2, are utilized to model the injection-moulding process.

In Section 3.2.1 restrictions with respect to the cavity geometries that will be considered are discussed. For generalized Newtonian material behaviour the simplified set of balance equations is given. Section 3.2.2 shows a derivation of the coupled pressure and temperature problem. This formulation is suitable for the analysis of complex shaped, thin-walled products, that are denoted $2 \frac{1}{2} \mathrm{D}$ geometries.

In Section 3.3 attention is focused on the calculation of flow-induced stresses, where both the compressible Leonov model and the compressible Wagner model are employed. A decoupled procedure is used, where it is assumed that the elasticity of the melt does not affect the flow kinematics to a great extent.

In Section 3.4 the calculation of thermal stresses, developing in the solidifying part of the product during moulding, is discussed. In this case the material behaviour is described with the linear thermo-viscoelastic model. 
Table 1. Constitutive equations for the Cauchy stress tensor

The compressible Leonov model

Section 2.1 .2

$$
\begin{aligned}
& \boldsymbol{\sigma}=-p \mathbf{I}+\boldsymbol{\sigma}_{e}^{d}+\boldsymbol{\sigma}_{p} \\
& \boldsymbol{\sigma}_{e}^{d}=\sum_{i=1}^{m} \frac{\eta_{i}}{\bar{\theta}_{i}} \overline{\mathbf{B}}_{e i}^{d}, \quad \sigma_{p}=2 \eta_{r} \mathbf{D}^{d} \\
& \nu=\nu(p, T) \\
& \mathbf{D}_{p i}=\frac{1}{4 \theta_{i}}\left(\overline{\mathbf{B}}_{e i}^{d}-\overline{\mathbf{B}}_{e i}^{-d}\right) \\
& \dot{\overline{\mathbf{B}}}_{e i}=\left(\mathbf{L}^{d}-\mathbf{D}_{p i}\right) \cdot \overline{\mathbf{B}}_{e i}+\overline{\mathbf{B}}_{e i} \cdot\left(\mathbf{L}^{d c}-\mathbf{D}_{p i}\right)
\end{aligned}
$$

The compressible Wagner model

Section 2.1.3

$$
\begin{aligned}
& \boldsymbol{\sigma}=-p(\rho(t)) \mathbf{I}+\int_{-\infty}^{t} M(t-\tau) h\left(\overline{\mathscr{I}}_{1}, \overline{\mathscr{I}}_{2}\right) \overline{\mathbf{C}}_{t}^{-d}(\tau) \mathrm{d} \tau \\
& \rho=\rho(p, T)
\end{aligned}
$$

The generalized Newtonian model

$\boldsymbol{\sigma}=-p \mathbf{I}+\boldsymbol{\sigma}^{d}$

$p=p_{0}-\mu \operatorname{tr}(\mathbf{D}), \quad \sigma^{d}=2 \eta \mathbf{D}^{d}$

$\mu=\mu\left(p_{0}, T, \dot{\rho}, I_{2}^{\mathbf{D}^{d}}, I_{3}^{\mathbf{D}^{d}}\right), \quad \eta=\eta\left(p_{0}, T, \dot{\rho}, I_{2}^{d}, I_{3}^{\mathbf{D}^{d}}\right)$

$\rho=\rho\left(p_{0}, T\right)$

The linear thermo-viscoelastic model Section 2.1.6

$$
\begin{aligned}
& p(t)=\int_{0}^{t}\left(\frac{\alpha}{\kappa} \dot{T}-\frac{1}{\kappa} \operatorname{tr}(\mathbf{D})\right) \mathrm{d} \tau \\
& \sigma^{d}=\sum_{i=1}^{m} 2 \int_{0}^{t} G_{i} e^{-(\xi(t)-\xi(\tau)) / \theta_{i 0}} \dot{\epsilon}^{d} \mathrm{~d} \tau, \quad G_{i}=\frac{\eta_{i 0}}{\theta_{i 0}} \\
& \xi(\tau)=\int_{0}^{\tau} \frac{1}{a_{T}} \mathrm{~d} s
\end{aligned}
$$

\subsection{Analysis of Compressible, Solidifying, Generalized Newtonian Flow}

\subsubsection{Thin Film Approximation}

In this section, geometrical considerations will result in a simplification of the total set of equations. In most practical situations the wall thickness of injection-moulded 


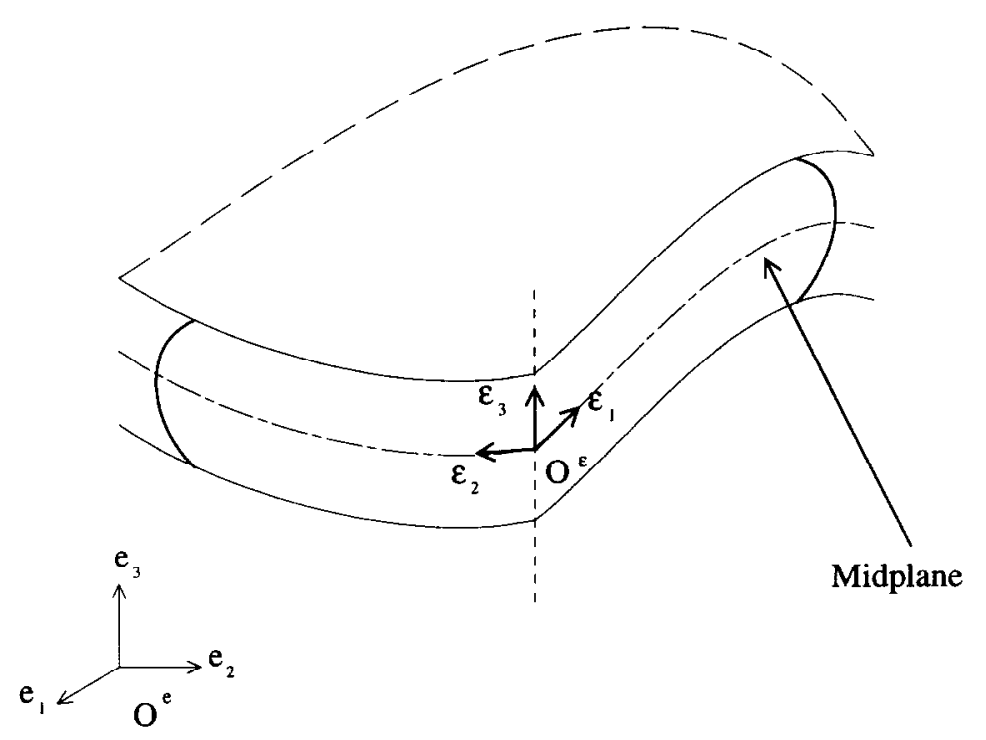

Fig. 1. Local base $O_{\epsilon}$.

products varies between 0.5 and $4 \mathrm{~mm}$. The thickness is bounded by a maximum value to avoid excessive cooling times and by a minimum value to prohibit premature stoppage of flow.

A restriction is made by only considering narrow, weakly curved channels. This means that the ratio of the cavity thickness $h$ and a characteristic length $l$, in the midplane of the cavity, must be much less than unity. As a consequence of this approximation the extra pressure drop caused by discontinuous changes in the cavity height or by corners is not taken into account.

In every point of the mid-plane a local Cartesian vector base is constructed $O_{\epsilon}:\left\{\vec{\epsilon}_{1}, \vec{\epsilon}_{2}, \vec{\epsilon}_{3}\right\}$; see Fig. 1. Note that $\vec{\epsilon}_{3}$ is normal with respect to the mid-plane. An arbitrary vector $\vec{a}$ can be decomposed in a vector $\vec{a}^{*}$ parallel to the mid-plane and a component in the direction of $\vec{\epsilon}_{3}$

$$
\overrightarrow{\mathrm{a}}=a_{i}^{\epsilon} \vec{\epsilon}_{i}=\overrightarrow{\mathrm{a}}^{*}+a_{3}^{\epsilon} \vec{\epsilon}_{3}
$$

The thin film approximation can be employed (e.g. Schlichting, ${ }^{84}$ Chapter vii; Richardson $;{ }^{79}$ Hieber and Shen; ${ }^{34}$ Sitters, ${ }^{87}$ Chapter 3; Boshouwers and van der Werf, ${ }^{8}$ Chapter 2; Flaman ${ }^{26}$ Chapter 3). Moreover, in the derivation of a simplified set of equations, use is made of the high viscosity of polymer melts. This implies that the Reynolds number (i.e. the ratio of the viscous forces and the stationary inertial forces), is low: $R e \ll 1$. The main results of the approximations mentioned are:

- The inertial and body forces that appear in the momentum equation (2) may be neglected with respect to the viscous forces.

- The pressure is independent of the $\vec{\epsilon}_{3}$ direction.

- Velocity gradients parallel to the mid-plane are small compared to the velocity gradients in the (perpendicular) $\vec{\epsilon}_{3}$ direction. 
- Velocity components in the $\vec{\epsilon}_{3}$ direction are small compared to those tangent to the mid-plane.

- Thermal conduction parallel to the mid-plane is negligible with respect to conduction in the $\vec{\epsilon}_{3}$ direction.

Since the mould is cooled below the solidification temperature, the polymer melt that comes into contact with the mould walls solidifies. From the mould walls solid layers grow inward. In these solid layers the velocity components parallel to the midplane are assumed to be zero, in contrast to the small non-zero velocity component in the $\vec{\epsilon}_{3}$ direction due to density changes caused by cooling. The position of the solidliquid interfaces is denoted as $s^{-} \vec{\epsilon}_{3}$ and $s^{+} \vec{\epsilon}_{3}$ respectively. The mould thickness is $h$.

With these simplifications the deviatoric part of the deformation rate tensor reduces to

$$
\mathbf{D}^{d}=1 / 2 \frac{\partial}{\partial x_{3}^{\epsilon}}\left(\vec{v}^{*} \vec{\epsilon}_{3}+\vec{\epsilon}_{3} \vec{v}^{*}\right)
$$

The shear rate $\dot{\gamma}$, defined as $\sqrt{2 \mathbf{D}^{d}: \mathbf{D}^{d}}$, is reduced to $\left|\partial \overrightarrow{\mathrm{v}}^{*} / \partial x_{3}^{\epsilon}\right|$.

Little is known about the magnitude of the bulk viscosity in polymer melts (see Batchelor, ${ }^{4}$ Section 3.4). The velocity gradients appearing in $\operatorname{tr}(\mathbf{D})$ are negligible compared to those in $\mathbf{D}^{d}$. In eq. (22) $)_{1}$, the term $\mu \operatorname{tr}(\mathbf{D}) \mathbf{I}$ is neglected in relation to $\eta \mathbf{D}^{d}$. As a consequence the thermodynamic pressure $p_{0}$ equals the hydrostatic pressure $p$.

3.2.1.1. Simplified Set of Equations - Substitution of these simplifications in the constitutive eqs (21)-(23), (40), (41) and subsequent substitution in the equations of balance, results in the following system of equations:

$$
\begin{gathered}
\vec{\nabla}^{*} \cdot \overrightarrow{\mathrm{v}}^{*}+\frac{\partial v_{3}^{\epsilon}}{\partial x_{3}^{\epsilon}}=-\frac{\dot{\rho}}{\rho}, \\
\vec{\nabla}^{*} p=\frac{\partial}{\partial x_{3}^{\epsilon}}\left(\eta \frac{\partial \overrightarrow{\mathrm{v}}^{*}}{\partial x_{3}^{\epsilon}}\right), \quad \frac{\partial p}{\partial x_{3}^{\epsilon}}=0, \\
\rho c_{p} \dot{T}=\frac{\partial}{\partial x_{3}^{\epsilon}}\left(\lambda \frac{\partial T}{\partial x_{3}^{\epsilon}}\right)+\eta \dot{\gamma}^{2}-\frac{T}{\rho}\left(\frac{\partial \rho}{\partial T}\right)_{p} \dot{p},
\end{gathered}
$$

where the mould is assumed to be rigid. This implies that the cavity dimensions are set and are not coupled with the pressure field. The mechanical and thermal behaviour of the mould is simplified considerably, and attention is focused on the product. In his calculations, Baaijens ${ }^{2}$ showed a substantial influence of the elasticity of the mould on the history of the cavity pressure during the holding and cooling stage, and consequently on both the flow-induced and thermal stresses. An efficient analysis of the thermo-mechanical behaviour of complex shaped moulds is not straightforward, but obviously needs to be a topic for future research.

The set of eqs (44)-(46) comprises five equations in which 5 unknowns - two scalars $p$, $T$ and three velocity components $\overrightarrow{\mathrm{v}}^{*}, v_{3}^{\epsilon}-$ appear. This system of equations is instationary, non-linear and coupled because the viscosity and density are temperature- and 


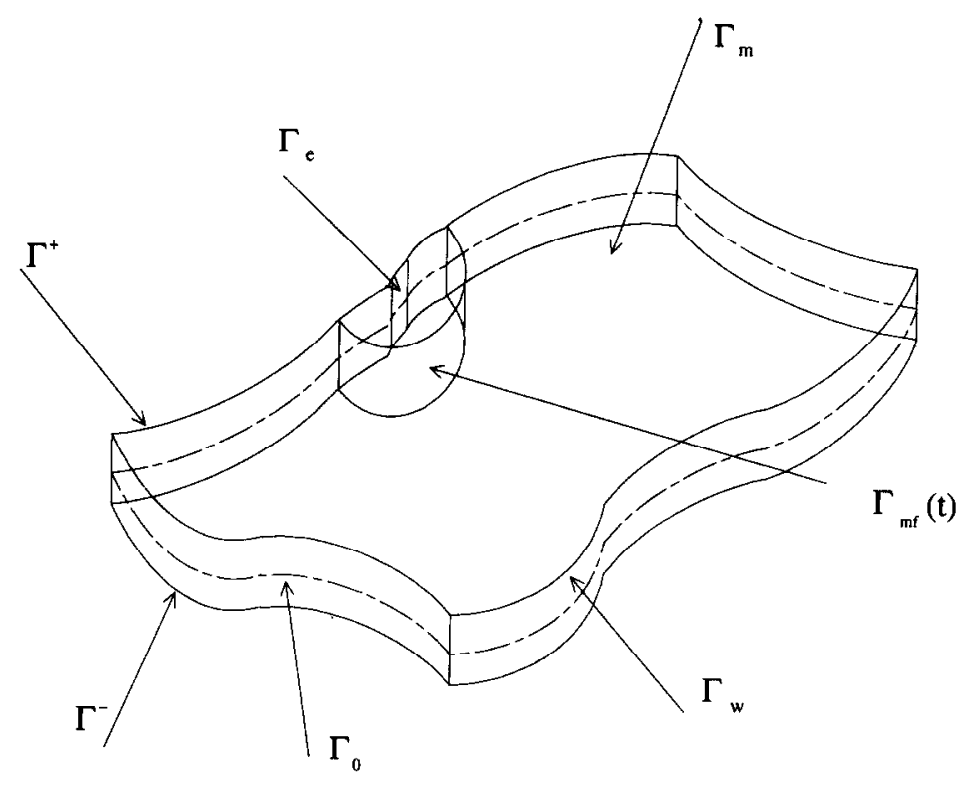

Fig. 2. Model of the mould cavity and its boundaries.

pressure-dependent. In the case of incompressible, isothermal flow the well known HeleShaw flow appears (Schlichting, ${ }^{84}$ Chapter vi.d; Hele-Shaw ${ }^{32}$ ).

\subsubsection{Pressure Problem}

In this section the so-called pressure problem is derived by eliminating the velocity components from eqs (44) and (45). This is attained by integration of these relations over the thickness, yielding an equation where pressure is the only unknown variable. This procedure is a result of eq. (45) 2 .

A thin cavity is considered, defining a region in space $\Omega$; see Fig. 2 . The upper and lower surface bounding the cavity are denoted $\Gamma^{+}$and $\Gamma^{-}$respectively. These boundaries are impenetrable and parallel to the mid-plane of the cavity $\Gamma_{0}$. The bounding planes that are perpendicular to the mid-plane $\Gamma_{0}$ are considered next. The part of this boundary that is impenetrable is denoted $\Gamma_{w}$.

At $t=0$ the process starts as the molten polymer flows into the mould cavity. The spots where the polymer enters the mould are denoted $\Gamma_{e}$. As time proceeds, a certain region of the mould cavity $\Omega_{p}(t)$ is occupied by the polymer. This region is bounded by parts of $\Gamma^{+}, \Gamma^{-}, \Gamma_{e}, \Gamma_{w}$ and the melt front $\Gamma_{m f}(t)$. The melt front is the moving boundary between the polymer and the air in the mould cavity. The outer surface of the mould at which a temperature may be prescribed, is denoted $\Gamma_{m}$.

The time interval of interest is $\mathscr{T}_{m}=\left[0, t_{e}\right]$, where at $t=t_{e}$ the product is released from the mould. It is assumed that at the cavity entry, the volume flux is prescribed during the time interval $\mathscr{T}_{Q}=\left[0, t_{s w}\right]$. At time $t_{s w}\left(<t_{e}\right)$, either the mould is completely filled or the maximum pressure is reached, depending on both the mould and the 
injection-moulding machine. For $t \in \mathscr{T}_{p}=\left[t_{s w}, t_{g f o}\right]$ the pressure at the cavity entrance is assumed to be known. At time $t_{g f o}$ the gate is completely solidified. For $t \in \mathscr{T}_{c}=\left[t_{\text {gfo }}, t_{e}\right]$ no material is allowed to enter the mould anymore. So the time interval $\mathscr{T}_{m}=\mathscr{T}_{Q} \cup \mathscr{T}_{p} \cup \mathscr{T}_{c}$.

The pressure problem can now be summarized as follows:

Problem 1 (PP): Given $T(\overrightarrow{\mathrm{x}}, t)$, find the pressure field $p(\overrightarrow{\mathrm{x}}, t) \geq 0$ such that

$$
\begin{aligned}
& \vec{\nabla}^{*} \cdot\left(S \vec{\nabla}^{*} p\right)-\int_{-h / 2}^{h / 2} \kappa \dot{p} \mathrm{~d} \chi=-\int_{-h / 2}^{h / 2} \alpha \dot{T} \mathrm{~d} \chi, \\
& S=J_{2}-\frac{J_{1}^{2}}{J_{0}}, \quad J_{i}=\int_{s^{-}}^{s^{+}} \frac{\chi^{i}}{\eta} \mathrm{d} \chi, \quad i=0,1,2
\end{aligned}
$$

subject to the boundary conditions mentioned above.

The pressure problem in case of viscoelastic behaviour according to the compressible Leonov constitutive equation can be summarized as follows (see Baaijens ${ }^{3}$ ).

Problem 2 (PPVE): Given $T(\overrightarrow{\mathrm{x}}, t)$, find the pressure field $p(\overrightarrow{\mathrm{x}}, t) \geq 0$ such that

$$
\begin{gathered}
\vec{\nabla}^{*} \cdot\left(S \vec{\nabla}^{*} p+\overrightarrow{\mathrm{u}}^{*}\right)-\int_{-h / 2}^{h / 2} \kappa \dot{p} \mathrm{~d} \chi=-\int_{-h / 2}^{h / 2} \alpha \dot{T} \mathrm{~d} \chi, \\
S=J_{2}-\frac{J_{1}^{2}}{J_{0}}, \quad J_{i}=\int_{s^{-}}^{s^{+}} \frac{\chi^{i}}{\eta} \mathrm{d} \chi, \quad i=0,1,2 \\
\overrightarrow{\mathrm{u}}^{*}=u_{j 3} \vec{\epsilon}_{j}, \quad u_{j 3}=\frac{J_{1}}{J_{0}} \int_{s^{-}}^{s^{+}} \frac{\sigma_{e_{j 3}}}{\eta} \mathrm{d} \chi-\int_{s^{-}}^{s^{+}} \frac{\chi \sigma_{e_{j 3}}}{\eta} \mathrm{d} \chi, \quad j=1,2 \\
\sigma_{e_{j 3}}=\sigma_{e}^{d}: \vec{\epsilon}_{j} \vec{\epsilon}_{3}=\sum_{k=1}^{m} \frac{\eta_{k}}{\theta_{k}} \overline{\mathbf{B}}_{e k}^{d}: \vec{\epsilon}_{j} \vec{\epsilon}_{3}, \quad j=1,2
\end{gathered}
$$

subject to the boundary conditions mentioned above.

\subsubsection{Temperature Problem}

For the temperature problem, boundary conditions must be specified on the cavity surfaces and at the entry of the cavity. Either a wall temperature is prescribed or a Biot type of boundary condition is given.

The temperature problem is defined as follows.

Problem 3 (TP): Given $p(\vec{x}, t)$, find the temperature field $T(\overrightarrow{\mathrm{x}}, t)$ such that

$$
\rho c_{p} \dot{T}=\frac{\partial}{\partial x_{3}^{\epsilon}}\left(\lambda \frac{\partial T}{\partial x_{3}^{\epsilon}}\right)+\eta \dot{\gamma}^{2}-\frac{T}{\rho}\left(\frac{\partial \rho}{\partial T}\right)_{p} \dot{p}
$$

subject to the boundary conditions mentioned above. 
The model defined above is referred to as a $2 \frac{1}{2} \mathrm{D}$ model. This name expresses the fact that the pressure field is two-dimensional, and the velocity and temperature fields are three-dimensional.

\subsection{Flow-Induced Stresses}

\subsubsection{Introduction}

In Section 3.2.2 the polymer was assumed to be purely viscous. Although this simplified fluid model proves to give good predictions of the velocity, pressure and temperature fields, it does not describe all phenomena that influence product properties. The main quantities that determine the properties, as well as the evolution of the dimensions and the shape, of injection-moulded products, are the frozen-in molecular orientation and the residual stresses. Residual stresses stem from two main sources. Firstly there are frozen-in flow-induced stresses (entropic stresses) and secondly thermally- and pressure-induced stresses (energy elastic stresses). Flow-induced stresses are the subject of this section, while thermally-induced stresses will be dealt with in Section 3.4.

Generally, the residual flow-induced stresses, caused by the orientation of polymer molecules in the direction of flow, are considerably smaller than the residual thermally-induced stresses. However, it is not possible to neglect the former, because the frozen-in orientation of polymer molecules is responsible for the anisotropy of mechanical, thermal and optical properties, and affects the long-term dimensional stability. Orientation and flow-induced stresses develop during the viscoelastic flow of the polymer in both the filling and the post-filling stage of the injection-moulding process. They develop in the fluid state, i.e. the temperature must be above the glass transition temperature $T_{g}$, and are accompanied with alignment of the chain molecules with respect to the flow direction. Complete relaxation of these stresses, and corresponding molecular orientation, is prevented by the fast solidification caused by high cooling rates prevailing in the injection-moulding process.

Employing a viscoelastic constitutive equation in numerical simulations is not feasible for complicated product geometries, on account of excessive computing time. Therefore a decoupled method to calculate the flow-induced stresses is proposed. The main assumption is that the elastic behaviour of the polymer melt only marginally influences the flow kinematics. Then the pressure problem (Problem 1) and the temperature problem (Problem 3) may be solved to provide the flow kinematics used to calculate the flow-induced stresses.

The so-called decoupled method was validated by Baaijens and Douven, ${ }^{3}$ for a simple mould geometry, a strip. They compared flow-induced stresses, calculated in a fully coupled way, with results obtained by the decoupled method. The constitutive equation used was the compressible version of the Leonov model (see Leonovi ${ }^{59}$ Baaijens; ${ }^{1}$ Flaman $^{26}$ ). Results of both calculations compared satisfactorily. The savings in computing time were substantial, a reduction of more than a factor of 10 being established, therefore the decoupled method is employed throughout this study. 
In the next sections, for simplicity, the elaboration of the theory for flow-induced stresses is demonstrated for one-dimensional flow geometries. The Leonov model is implemented for $2 \frac{1}{2} \mathrm{D}$ geometries as well.

\subsubsection{Decoupled Method: The Compressible Leonov Model}

In this section the decoupled method is discussed, using the compressible Leonov model, while Section 3.3.3 deals with the application of the compressible Wagner model.

Solving the coupled pressure problem (Problem 1) and temperature problem (Problem 3) yields the pressure, velocity and temperature fields in the case of purely viscous melt behaviour. These fields are substituted in the Leonov constitutive equation (7)-(11). The specific volume $\nu$ can be obtained by evaluating the Tait equation (37) for the pressure and temperature obtained by solving the pressure and tempcraturc problcm. The term $\sigma_{p}$ can be calculated by combining the retardation viscosity $\eta_{r}$ and the velocity gradient field $\mathbf{D}^{d}$.

Calculation of the elastic part of the stresses $\boldsymbol{\sigma}_{e}^{d}$ will take some more effort. Equations (10) and (11) are combined and supply a system of non-linear partial differential equations for the components of each Finger strain tensor $\overline{\mathbf{B}}_{e i}$. Obviously eq. $(5)_{2}$ and the definition of $\overline{\mathbf{B}}_{e i}$ according to $(6)_{3}$ yield $\operatorname{det}\left(\overline{\mathbf{B}}_{e i}\right)=1$. Thus the decoupled Leonov problem is defined as follows:

Problem 4 (DLP): Given $T(\overrightarrow{\mathrm{x}}, t)$, and a velocity gradient field $\mathbf{L}(\overrightarrow{\mathrm{x}}, t)$ find the Finger strain tensors for $i=1, \ldots, m, \overline{\mathbf{B}}_{e i}(\overrightarrow{\mathrm{x}}, t)$ such that for $i=1, \ldots, m$

$$
\begin{gathered}
\dot{\overline{\mathbf{B}}}_{e i}=\mathbf{L}^{d} \cdot \overline{\mathbf{B}}_{e i}+\overline{\mathbf{B}}_{e i} \cdot \mathbf{L}^{d c}-\frac{1}{2 \theta_{i}}\left(\overline{\mathbf{B}}_{e i} \cdot \overline{\mathbf{B}}_{e i}-\mathbf{I}\right)+\frac{1}{6 \theta_{i}}\left(\operatorname{tr}\left(\overline{\mathbf{B}}_{e i}\right)-\operatorname{tr}\left(\overline{\mathbf{B}}_{e i}^{-1}\right)\right) \overline{\mathbf{B}}_{e i}, \\
\operatorname{det}\left(\overline{\mathbf{B}}_{e i}\right)=1,
\end{gathered}
$$

where the initial condition is governed by isothermal, stationary flow.

\subsubsection{Decoupled Method: The Compressible Wagner Model}

The solutions of the pressure problem (Problem 1) and the temperature problem (Problem 3) are substituted in the compressible version of the Wagner model according to eq. (12). The specific volume $\nu=1 / \rho$ is modelled by the Tait equation (37). The memory function $M$ according to eq. (20) is evaluated by inserting the known temperature history. The damping function $h$, according to eq. (15), is used.

Only one-dimensional flow is considered and only shear flow is taken into account, thus

$$
\mathbf{L}=\dot{\gamma} \overrightarrow{\mathrm{e}}_{1} \overrightarrow{\mathrm{e}}_{3}, \quad \dot{\gamma}=\frac{\partial v_{1}}{\partial x_{3}}
$$

The decoupled Wagner problem (1D) is defined as follows: 
Problem 5 (DWP-1D): Given $T(\overrightarrow{\mathrm{x}}, t)$, and a shear rate field $\dot{\gamma}(\overrightarrow{\mathrm{x}}, t)$ find the shear stress $\sigma_{13}(\overrightarrow{\mathrm{x}}, t)$ and the first normal stress difference $N_{1}(\overrightarrow{\mathrm{x}}, t)$ such that

$$
\begin{gathered}
\sigma_{13}=\int_{-\infty}^{t} M(t-\tau, T) h\left(3+\left\{\int_{\tau_{1}}^{t} \dot{\gamma} \mathrm{d} t^{\prime}\right\}\right)\left[\int_{\tau_{1}}^{t} \dot{\gamma} \mathrm{d} t^{\prime}\right] \mathrm{d} \tau \\
N_{1}=\sigma_{11}-\sigma_{33}=\int_{-\infty}^{t} M(t-\tau, T) h\left(3+\left\{\int_{\tau_{1}}^{t} \dot{\gamma} \mathrm{d} t^{\prime}\right\}^{2}\right)\left[\int_{\tau_{1}}^{t} \dot{\gamma} \mathrm{d} t^{\prime}\right]^{2} \mathrm{~d} \tau \\
\tau_{1}= \begin{cases}\tau & \text { if } \tau>0 \\
0 & \text { if } \tau \leq 0\end{cases}
\end{gathered}
$$

where the initial condition is governed by isothermal, stationary flow.

\subsection{Thermally- and Pressure-Induced Stresses}

\subsubsection{Introduction}

Thermally-induced stresses develop in an injection-moulded product during cooling, both inside the mould and after demoulding. An inhomogeneous temperature field develops in the moulded polymer, mainly as a consequence of its low thermal conductivity and the difference between injection temperature and mould temperature. This inhomogeneous temperature field, with a hot and fluid core combined with layers at the mould walls which are already solidified, causes material points to cool from above to below $T_{g}$ at different times. Thus the material experiences differential shrinkage, causing thermal stresses. These stresses are pronounced because the modulus rises several orders of magnitude as the material cools down to below $T_{g}$.

The development of thermal stresses is not only influenced by the temperature history, but also by the pressure field. The cavity pressure is responsible for the contact between the moulded product and the mould surface. If the pressure drops to zero the product will have no other kinematic restraint but the mould geometry to prevent shrinkage.

The residual thermally-induced stresses are frequently an order of magnitude larger than the frozen-in flow-induced stresses.

\subsubsection{Linear Thermo-Viscoelastic Modelling of Thermal Stresses}

3.4.2.1. Basic Assumptions - In order to calculate the thermal stresses developing in solid layers, the linear thermo-viscoelastic theory is employed (see Section 2.1.6). By the use of this geometrical linear theory, small displacement gradients are implied. A number of additional assumptions are provided below (see also Baaijens ${ }^{2}$ ).

1. All assumptions employed in defining the pressure problem (Problem 1) and the temperature problem (Problem 3) are valid. The temperature field and the pressure field are used as input for the calculation of the thermal stresses. The 
stress component $\sigma_{33}^{\epsilon}$ equals minus the melt pressure for as long as the temperature in the mid-plane $T^{*}$ is greater than the glass transition temperature.

2. As long as the melt pressure is non-zero in a point of the mid-plane $\Gamma_{0}$, the material sticks to the mould walls in this point. So global displacements are zero. The only non-zero strain component is $\epsilon_{33}^{\epsilon}$.

3. If the pressure drops to zero in a point of $\Gamma_{0}$, the material is allowed to loose contact from the mould walls.

4. The gap between product surfaces and mould walls that may develop if the pressure is zero, will influence the transport of heat from the product to its environment. In the present analysis this effect is not yet taken into account. This restriction can easily be overcome, once experimental data are available.

5. Flow-induced anisotropy is not considered; isotropic material behaviour is assumed.

6. Warpage is taken into account, only after the product is ejected from the mould. Thus, when the product is still in the mould, a membrane formulation is employed, and when the product is released from the mould, a shell formulation is employed.

7. In-plane deformation is allowed for in the analysis. However, for complex shaped products the in-plane shrinkage, in one or two directions, may be prohibited by the geometry of the mould. In this case the appropriate kinematic boundary conditions suppressing these displacements should be employed. Formulating the right set of boundary conditions for a given geometry is not trivial.

\subsubsection{Numerical Solution of the Thermal Stress Problem}

The thermal stress problem solves the equilibrium equation $\vec{\nabla} \cdot \sigma=\overrightarrow{0}$, for a given pressure and temperature field, applying the correct boundary conditions.

Now the numerical strategy to compute the thermally-induced stresses will be outlined.

3.4.3.1. Incremental Formulation of the Linear Thermo-Viscoelastic Model - An incremental formulation for the linear thermo-viscoelastic constitutive equation is given (see Baaijens ${ }^{2}$ ). Temporal discretization is adopted. The stress according to the linear thcrmo-viscoelastic model as expressed by eqs (33) and (34), at $t=t_{n}$, reads

$$
\begin{gathered}
\sigma_{n}=-p_{n}^{h} \mathbf{I}+\sum_{i=1}^{m} \boldsymbol{\sigma}_{i_{n}}^{d} \\
p_{n}^{h}=\int_{0}^{t_{n}}\left(\frac{\alpha}{\kappa} \dot{T}-\frac{1}{\kappa} \operatorname{tr}(\dot{\boldsymbol{\epsilon}})\right) \mathrm{d} \tau \\
\boldsymbol{\sigma}_{i_{n}}^{d}=2 \int_{0}^{t_{n}} G_{i} e^{-\left(\xi_{n}-\xi(\tau)\right) / \theta_{i 0}} \dot{\boldsymbol{\epsilon}}^{d} \mathrm{~d} \tau
\end{gathered}
$$

Note that a subscript $n$ indicates evaluation at time $t_{n}$. The state at $t_{n}$ is completely determined. Next the stress state at $t_{n+1}$ can be evaluated, provided that the temperature and strain fields are known. An incremental formulation for the stresses is 
derived, where only values at $t_{n}$ need to be known. The following incremental variables are introduced:

$$
\Delta \xi_{n+1}=\xi_{n+1}-\xi_{n}, \quad \Delta T_{n+1}=T_{n+1}-T_{n}, \quad \Delta \epsilon_{n+1}-\epsilon_{n+1}-\epsilon_{n}
$$

It is assumed that $\epsilon$ and $T$ vary linearly between two discrete times, implying that $\dot{\epsilon}$ and $\dot{T}$ are constant over each time increment, thus yielding

$$
\dot{\epsilon}=\frac{\Delta \epsilon_{n+1}}{\Delta t_{n+1}}, \quad \dot{T}=\frac{\Delta T_{n+1}}{\Delta t_{n+1}} \quad \text { for } t \in\left[t_{n}, t_{n+1}\right]
$$

After extensive rewriting of eq. (60) for $t=t_{n+1}$, the following expression is derived for $\boldsymbol{\sigma}$

$$
\sigma_{n+1}=\boldsymbol{\sigma}^{\cdot}+K^{\cdot} \operatorname{tr}\left(\Delta \epsilon_{n+1}\right) \mathbf{I}+2 G^{*} \Delta \boldsymbol{\epsilon}_{n+1}^{d}
$$

where

$$
\begin{gathered}
\boldsymbol{\sigma}^{\cdot}=-p_{n}^{h} \mathbf{I}-\beta^{\cdot} \Delta T_{n+1} \mathbf{I}+\sum_{i=1}^{m} e^{-\Delta \xi_{n+1} / \theta_{i 0}} \sigma_{i_{n}}^{d}, \\
K^{*}=\frac{1}{\Delta t_{n+1}} \int_{t_{n}}^{t_{n+1}} \frac{1}{\kappa} \mathrm{d} \tau, \\
G^{*}=\frac{1}{\Delta t_{n+1}} \sum_{i=1}^{m} \int_{t_{n}}^{t_{n+1}} \frac{\eta_{i 0}}{\theta_{i 0}} e^{-\left(\xi_{n+1}-\xi(\tau)\right) / \theta_{i 0}} \mathrm{~d} \tau \\
\beta^{\cdot}=\frac{1}{\Delta t_{n+1}} \int_{t_{n}}^{t_{n+1}} \frac{\alpha}{\kappa} \mathrm{d} \tau
\end{gathered}
$$

The quantities $\sigma^{*}, K^{*}, G^{*}$ and $\beta^{*}$ can be evaluated, when the state at $t_{n}$ is determined and the temperature history is determined up until $t_{n+1}$.

The stress components of the linear thermo-viscoelastic model are written with respect to the local base $O_{\epsilon}$. The incremental strain component $\Delta \epsilon_{33}$ is eliminated by means of

$$
\Delta \epsilon_{33}=\frac{\sigma_{33}-\sigma_{33}^{*}}{a}, \quad a=\frac{3 K^{*}+4 G^{*}}{3}
$$

Equation (65) with respect to the local base yields

$$
\begin{aligned}
& \underline{\sim}_{n+1}=\underline{M} \underset{\sim}{\Delta} \epsilon+\underline{g} \\
& {\underset{\sim}{\sigma}}^{T}=\left[\begin{array}{lllll}
\sigma_{11} & \sigma_{22} & \sigma_{12} & \sigma_{23} & \sigma_{13}
\end{array}\right], \\
& \Delta \epsilon^{T}=\left[\begin{array}{lllll}
\Delta \epsilon_{11} & \Delta \epsilon_{22} & 2 \Delta \epsilon_{12} & 2 \Delta \epsilon_{23} & 2 \Delta \epsilon_{13}
\end{array}\right] \\
& \underline{M}=\left[\begin{array}{ccccc}
a^{*} & b^{*} & 0 & 0 & 0 \\
b^{\cdot} & a^{*} & 0 & 0 & 0 \\
0 & 0 & G^{\cdot} & 0 & 0 \\
0 & 0 & 0 & G^{*} & 0 \\
0 & 0 & 0 & 0 & G^{\cdot}
\end{array}\right]
\end{aligned}
$$




$$
\begin{aligned}
& \underset{\sim}{g}=\left[\begin{array}{c}
\sigma_{11}^{\cdot}+\frac{b}{a}\left(\sigma_{33}-\sigma_{33}^{*}\right) \\
\sigma_{22}^{\cdot}+\frac{b}{a}\left(\sigma_{33}-\sigma_{33}^{\dot{*}}\right) \\
\sigma_{12}^{\cdot} \\
\sigma_{23}^{\cdot} \\
\sigma_{13}^{\cdot}
\end{array}\right] \\
& b=\frac{3 K^{*}-2 G^{*}}{3}, \quad a^{*}=a-\frac{b^{2}}{a}, \quad b^{\cdot}=b-\frac{b^{2}}{a}
\end{aligned}
$$

3.4.3.2. Boundary Conditions - In each point of the midsurface $\Gamma_{0}$, four different situations must be considered:

1. The pressure is non-zero and the temperature in the midsurface, $T^{*}$, is still above the glass transition temperature $T_{g}$ :

$$
p\left(=-\sigma_{33}\right) \geq 0, \quad T^{*} \geq T_{g}(p)
$$

2. The pressure is non-zero and the temperature in the midsurface, $T^{*}$, is below the glass transition temperature $T_{g}$ :

$$
p \geq 0, \quad T^{*}<T_{g}(p)
$$

3. The pressure is zero, but the product is still inside the mould:

$$
p=0, \quad t<t_{d e}
$$

4. The pressure is zero, and the product is ejected:

$$
p=0, \quad t \geq t_{d e}
$$

These four situations will be analyzed in more detail.

3.4.3.2.1. Constrained quench with fluid core - All strain components are zero, for $x_{3} \in[-h / 2, h / 2]$, except $\epsilon_{33}$ must obey $\int_{-h / 2}^{h / 2} \epsilon_{33} \mathrm{~d} x_{3}=0$. The non-zero stress components are evaluated by (see eqs (71)-(76)):

$$
\sigma_{33}=-p, \quad \sigma_{11}=\sigma_{22}=\sigma_{11}^{\cdot}+\frac{b}{a}\left(-p-\sigma_{33}^{\cdot}\right)
$$

3.4.3.2.2. Constrained quench with solid core - Identical to case 1, however, the nonzero stress components are now evaluated by (see eqs (71)-(76)):

$$
\begin{gathered}
\sigma_{33}=\frac{\int_{-h / 2}^{h / 2}\left(\sigma_{33}^{\cdot} / a\right) \mathrm{d} x_{3}}{\int_{-h / 2}^{h / 2}(1 / a) \mathrm{d} x_{3}}, \\
\sigma_{11}=\sigma_{22}=\sigma_{11}^{\cdot}+\frac{b}{a}\left(\sigma_{33}-\sigma_{33}^{\cdot}\right)
\end{gathered}
$$


In cases 1 and 2 no calculation of the displacements is required, because they are all prescribed to be zero.

3.4.3.2.3. Free quench in the mould - The only zero strain components are the shear strains $\epsilon_{13}$ and $\epsilon_{23}$, because bending is assumed to be prevented by the mould that envelops the product. Stress components are calculated with eqs (71)-(76).

3.4.3.2.4. Free quench, demoulded - All strain components may be non-zero and stresses are evaluated by means of eqs (71)-(76).

In cases 3 and 4 the displacement field is approximated by means of a finite element calculation, where the boundary conditions are represented by zero tractions on the surfaces.

For this purpose a shell element has been developed.

3.4.3.3. Element Formulation - The spatial discretization is performed by use of the finite element method. The displacement field is solved for each discrete time. The element used is a shell element, based on Mindlin plate theory where transverse shear strains are not assumed to be zero (see Hughes and Liu; ${ }^{36}$ Hughes, ${ }^{37}$ Chapter 6; Hughes and Tezduyan; ${ }^{38}$ Dvorkin and Bathe; ${ }^{23}$ Bathe and Dvorkin ${ }^{5}$ ). The element is a four-node bilinear isoparametric element, having 5 degrees of freedom per node. An extensive description of the element formulation is given in Douven. ${ }^{22}$

Remark 3.1. The midsurface of the shell elements coincide initially with the midsurface of the mould cavity $\Gamma_{0}$. As a consequence, the nodes of the triangular mesh used for solving the pressure problem and the nodes of the mesh of quadrilateral shells are identical.

Remark 3.2. The nodal values of the pressure and the temperature are solved from the pressure problem and the temperature problem, respectively. The values in the integration points are determined from these values by bilinear interpolation in the midsurface $\Gamma_{0}$. When the pressure is non-zero in all four nodes, all degrees of freedom of the corresponding element are set to zero. When the pressure is zero in all nodes, the element contributes to the global set of equations. Finally, when one to three nodes have a non-zero pressure value, these nodal degrees of freedom are set to zero by constraints, utilizing the penalty function method (Hughes, ${ }^{37}$ Chapter 4).

\subsection{Conclusion}

In this section, the theories derived in Section 2 were applied in modelling the injection-moulding process. This involved a simplification of the balance equations due to geometrical considerations, leading to a so-called $2 \frac{1}{2} \mathrm{D}$ model. Theories for calculating the polymer flow during the entire production cycle, as well as for the development of orientation, and flow-induced, and thermally-induced stresses, were derived and summarized in a number of well defined problems. 
Table 2. Model parameters in constitutive models

\begin{tabular}{cccc}
\hline $\begin{array}{c}\text { Leonov } \\
\text { Section 4.2.1 }\end{array}$ & $\begin{array}{c}\text { Wagner } \\
\text { Section 4.2.1-2 }\end{array}$ & $\begin{array}{c}\text { Lin. th.-viscoel. } \\
\text { Section 4.2.5 }\end{array}$ & $\begin{array}{c}\text { Gen. Newt. } \\
\text { Section 4.2.4 }\end{array}$ \\
\hline $\begin{array}{c}h\left(\overline{\mathscr{I}}_{1}, \overline{\mathscr{I}}_{2}\right) \\
\eta_{i 0}, \theta_{i 0}, \eta_{\mathrm{r} 0}\end{array}$ & $\eta_{i 0} / \theta_{i 0}, \theta_{i 0}$ & $G_{i}, \theta_{i 0}$ & $h\left(\bar{\Phi}_{1}, \overline{\mathscr{I}}_{2}\right)$ \\
melt & melt & glass & $G_{i}=\eta_{i 0} / \theta_{i 0}, \theta_{i 0}$ \\
$c_{1}, c_{2}, T_{0}$ & $c_{1}, c_{2}, T_{0}$ & & $c_{1}, c_{2}, T_{0}$ \\
\hline
\end{tabular}

\section{MATERIAL CHARACTERIZATION}

\subsection{Introduction}

In this Section, the material properties of two amorphous polymers, a polystyrene (PS 678E from DOW Chemical), and a polycarbonate (PC Makrolon CD 2000 from Bayer), are described.

In Table 2 an inventory is made of the data required by the constitutive models for the Cauchy stress tensor presented in Section 2. For the Wagner model the memory function $M$ is used (or the relaxation modulus $G$ ) according to eq. (20), representing multi mode Maxwell behaviour, and the damping function according to eq. (15) is employed. It is clear from this table that the linear viscoelastic properties in the melt state must be determined for both the Leonov and the Wagner model (see Section 4.2.1). In case of the Wagner model, the damping function must be determined independently (Section 4.2.2), although the stationary shear viscosity could be approximated from the linear viscoelastic data by means of the Cox-Merz rule. A comparison of the constitutive equations used is made in Section 4.2.3, and some separate steady state shear measurements are reported in Section 4.2.4. In Section 4.2.5, the linear viscoelastic data in the glass are presented. The $p \nu T$-data and the thermal properties are discussed in Section 4.3.

\subsection{Rheological Characterization}

\subsubsection{Linear Viscoelastic Measurements in the Melt}

PS $678 \mathrm{E}$ was measured at temperatures between $393 \mathrm{~K}$ and $538 \mathrm{~K}$ in the parallel plate geometry. The frequency range was $\omega \in\left[10^{-1}, 500\right] \mathrm{rad} / \mathrm{s}$. Figure 3 shows the loss angle against frequency for all temperatures. Thermo-rheological simple material behaviour is assumed (see Section 2.1.5), so the curves may be shifted horizontally along the frequency axis onto a master curve at $T_{0}=462 \mathrm{~K}$. Figure 4 shows $G_{d}$, where the curves are also shifted vertically. The horizontal and vertical shift factors, $a_{T}$ and $b_{T}$ respectively, are temperature-dependent and defined by Ferry, ${ }^{25}$ Chapter 11 :

$$
\begin{gathered}
\delta(\log \omega, T)=\delta\left(\log \omega a_{T}\left(T, T_{0}\right), T_{0}\right), \\
G_{d}(\log \omega, T)=b_{T}\left(T, T_{0}\right) G_{d}\left(\log \omega a_{T}\left(T, T_{0}\right), T_{0}\right)
\end{gathered}
$$




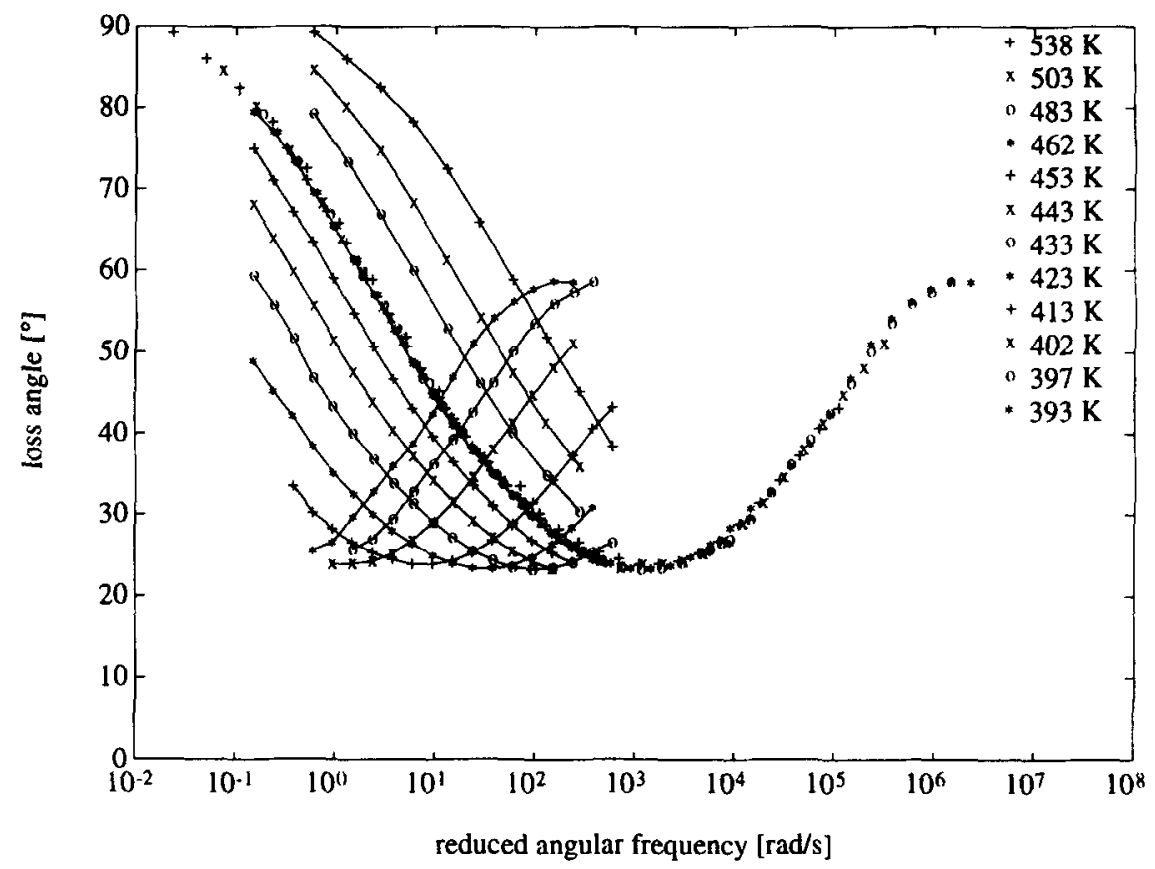

Fig. 3. Loss angle against angular frequency at various temperatures for PS 678E. The reference temperature of the master curve is $462 \mathrm{~K}$.

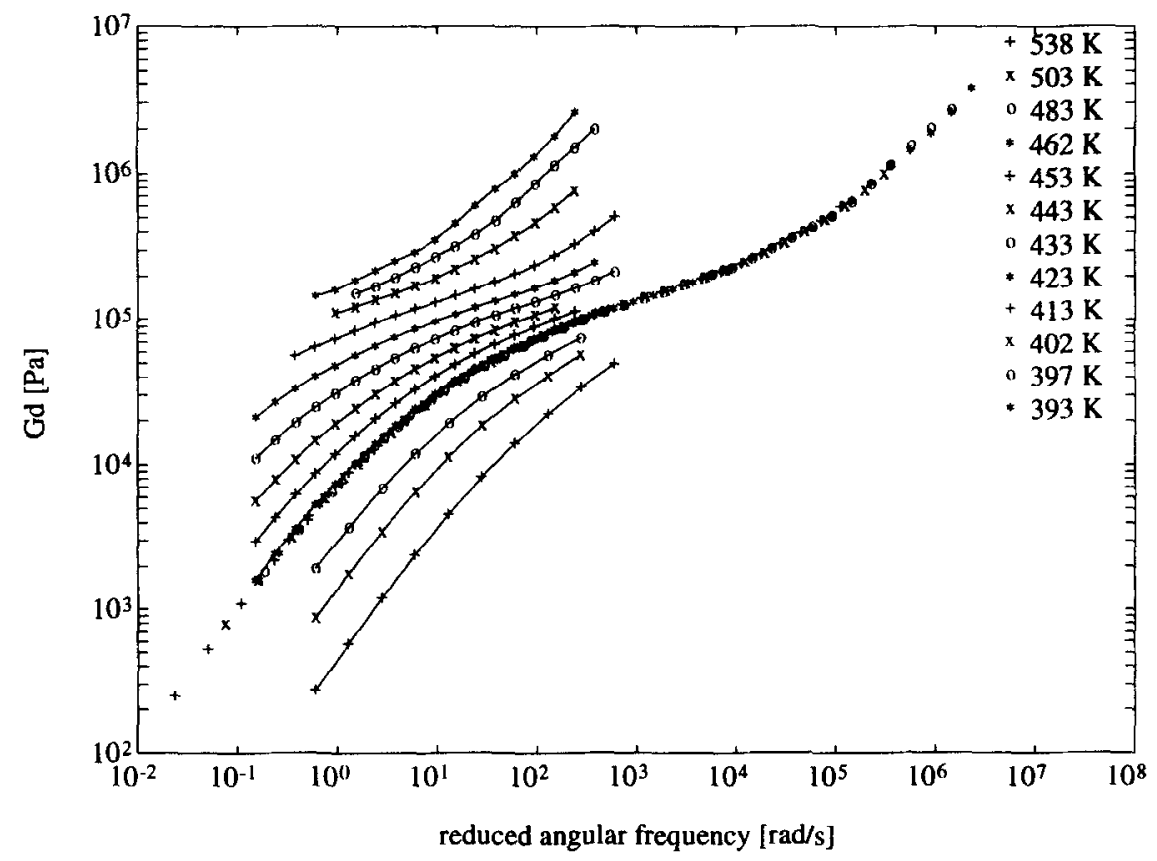

Fig, 4. Dynamic modulus against angular frequency at various temperatures for PS 678E. The reference temperature of the master curve is $462 \mathrm{~K}$. 


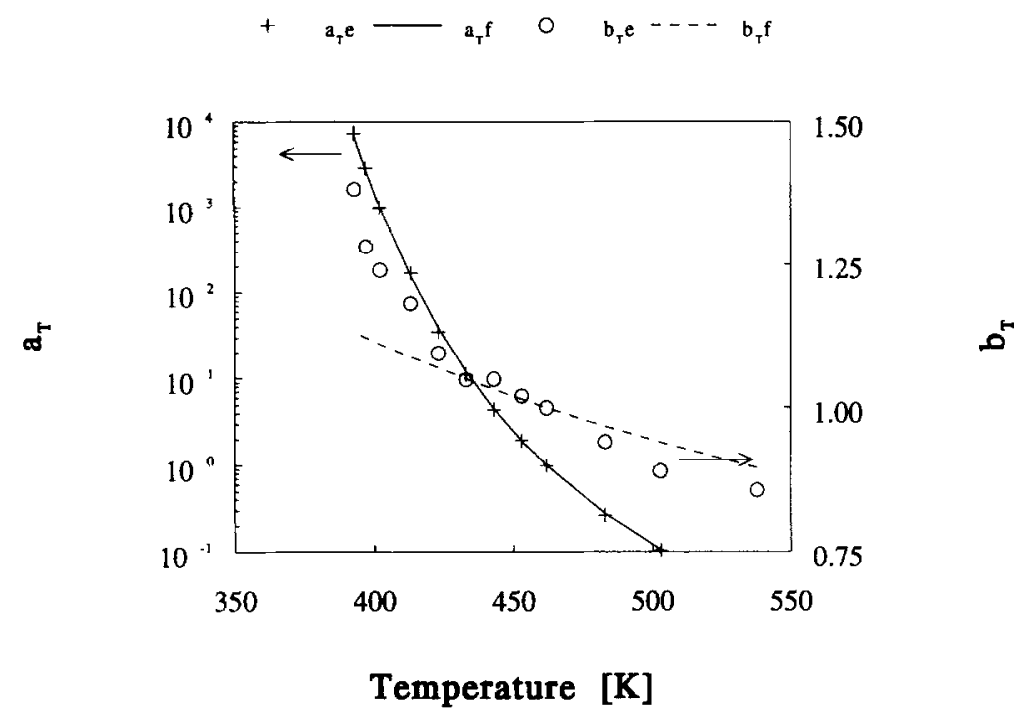

Fig. 5. Horizontal shift $a_{T}$ (+: experiment, --: WLF), and vertical shift $b_{T}$ (O: experiment, - - : temperature density correction) against temperature for PS 678E.

Figure 5 shows the horizontal and vertical shift factors as a function of temperature. In the melt a WLF-shift (eq. (31)) can be employed (see Table 3). The vertical shift factor in the melt state is modelled by $\rho T / \rho_{0} T_{0}$. This curve is also plotted in Fig. 5 and deviates considerably from the measured curve.

The master curve of PS $678 \mathrm{E}$ at $T_{0}=462 \mathrm{~K}$ is fitted using seven modes for the Wagner model and six modes and a retardation viscosity for the Leonov model. The master curve of PC Makrolon CD 2000 at $T_{0}=511 \mathrm{~K}$ is fitted using six modes for the Wagner model and six modes and a retardation viscosity for the Leonov model. The results are listed in Tables 4 and 5 respectively.

\subsubsection{The Damping Function}

The damping function can be determined in a number of ways. Wagner ${ }^{100}$ fitted the damping function according to eq. (15) with $m=0$, in shear flow. For a start-up constant shear flow, the shear strain $\gamma_{s}$, where the shear stress reaches a maximum value, and the shear strain $\gamma_{n}$, where the first normal stress difference reaches a maximum value, were used. Laun ${ }^{54}$ used steady state shear viscosity data and shear stress relaxation experiments to determine the parameters in the damping function.

Table 3. WLF parameters

\begin{tabular}{lcc}
\hline & PS 678E & PC Makrolon CD 2000 \\
\hline$c_{1}$ & 4.54 & 3.05 \\
$c_{2} \mathrm{~K}$ & 150.36 & 134.72 \\
$T_{0} \mathrm{~K}$ & 462 & 511 \\
\hline
\end{tabular}


Table 4. Linear viscoelastic parameters for PS 678E

\begin{tabular}{ccccc}
\hline Mode no. & \multicolumn{3}{c}{ Leonov } & \multicolumn{3}{c}{ Wagner } \\
\hline & \multicolumn{3}{c}{$\eta_{r 0}=2.05$ Pas } \\
$i$ & $\theta_{i 0} \quad s$ & $\eta_{i 0}$ Pas & $\theta_{i 0} s$ & $\eta_{i 0}$ Pas \\
1 & 2.25 & $6.01 \cdot 10^{3}$ & 3.7 & $3.55 \cdot 10^{3}$ \\
2 & $1.96 \cdot 10^{-1}$ & $5.64 \cdot 10^{3}$ & $3.37 \cdot 10^{-1}$ & $6.80 \cdot 10^{3}$ \\
3 & $1.17 \cdot 10^{-2}$ & $7.40 \cdot 10^{2}$ & $2.37 \cdot 10^{-2}$ & $1.3 \cdot 10^{3}$ \\
4 & $7.87 \cdot 10^{-4}$ & $6.5 \cdot 10^{1}$ & $1.95 \cdot 10^{-3}$ & $1.29 \cdot 10^{2}$ \\
5 & $5.47 \cdot 10^{-5}$ & 8.62 & $1.36 \cdot 10^{-4}$ & $1.74 \cdot 10^{1}$ \\
6 & $4.14 \cdot 10^{-6}$ & 2.33 & $1.16 \cdot 10^{-5}$ & 2.83 \\
7 & & & $7.18 \cdot 10^{-7}$ & 2.87 \\
\hline
\end{tabular}

In the present research, the following procedure was followed. For PS, start-up shear experiments were done on a Rheometrics Mechanical Spectrometer RMS-800 at DSM Research, The Netherlands. These experiments were performed with the cone-plate geometry at the reference temperature $T_{0}=462 \mathrm{~K}$ for shear rates: $\dot{\gamma}=0.2,0.4,1,2$, and $4 \mathrm{~s}^{-1}$, up to a shear strain value of 20 . The measured start-up shear viscosity and primary normal stress coefficient (see eq. (85)) are shown in Figs 6 and 7 respectively. The damping function was fitted on the start-up primary normal stress coefficient data. The fitted curves are seen in Figs 6 and 7 as well. The parameters found by this fit are listed in Table 6 . For PC, steady state data of the shear viscosity were used to fit the damping function. The results are listed in Table 6.

Remark 4.1. Since only shear flow data were used to fit the damping function, the parameter $\alpha$ in eq. (15) cannot be determined.

\subsubsection{Comparison of the Leonov and Wagner Model}

Following previous studies by Upadhyay ${ }^{97}$ and Larson, ${ }^{53}$ the behaviour of the Wagner and the Leonov model are compared for the cases of a start-up shear flow and a start-up elongational flow.

4.2.3.1. Start-up shear flow - A start-up flow is considered for various constant shear rates $\dot{\gamma}_{0}$. To obtain the results for the Leonov model the DLP must be solved

Table 5. Linear viscoelastic parameters for PC Makrolon CD 2000

\begin{tabular}{ccccc}
\hline Mode no. & \multicolumn{3}{c}{ Weonov } \\
\hline & \multicolumn{4}{c}{$\eta_{\text {ro }}=0.678$ Pas } \\
$i$ & $\theta_{i} s$ & $\eta_{i}$ Pas & $\theta_{i} s$ & $\eta_{i} \quad$ Pas \\
1 & $9.24 \cdot 10^{-3}$ & $3.10 \cdot 10^{2}$ & $5.64 \cdot 10^{-3}$ & $2.85 \cdot 10^{2}$ \\
2 & $9.55 \cdot 10^{-4}$ & $2.6 \cdot 10^{2}$ & $1.00 \cdot 10^{-3}$ & $2.70 \cdot 10^{2}$ \\
3 & $1.85 \cdot 10^{-4}$ & $6.85 \cdot 10^{1}$ & $1.78 \cdot 10^{-4}$ & $6.22 \cdot 10^{1}$ \\
4 & $4.82 \cdot 10^{-5}$ & $1.13 \cdot 10^{1}$ & $3.18 \cdot 10^{-5}$ & $1.39 \cdot 10^{1}$ \\
5 & $1.80 \cdot 10^{-5}$ & 4.25 & $5.65 \cdot 10^{-6}$ & $2.97 \cdot 10^{-1}$ \\
6 & $2.02 \cdot 10^{-6}$ & 1.38 & $1.00 \cdot 10^{-6}$ & 1.52 \\
\hline
\end{tabular}




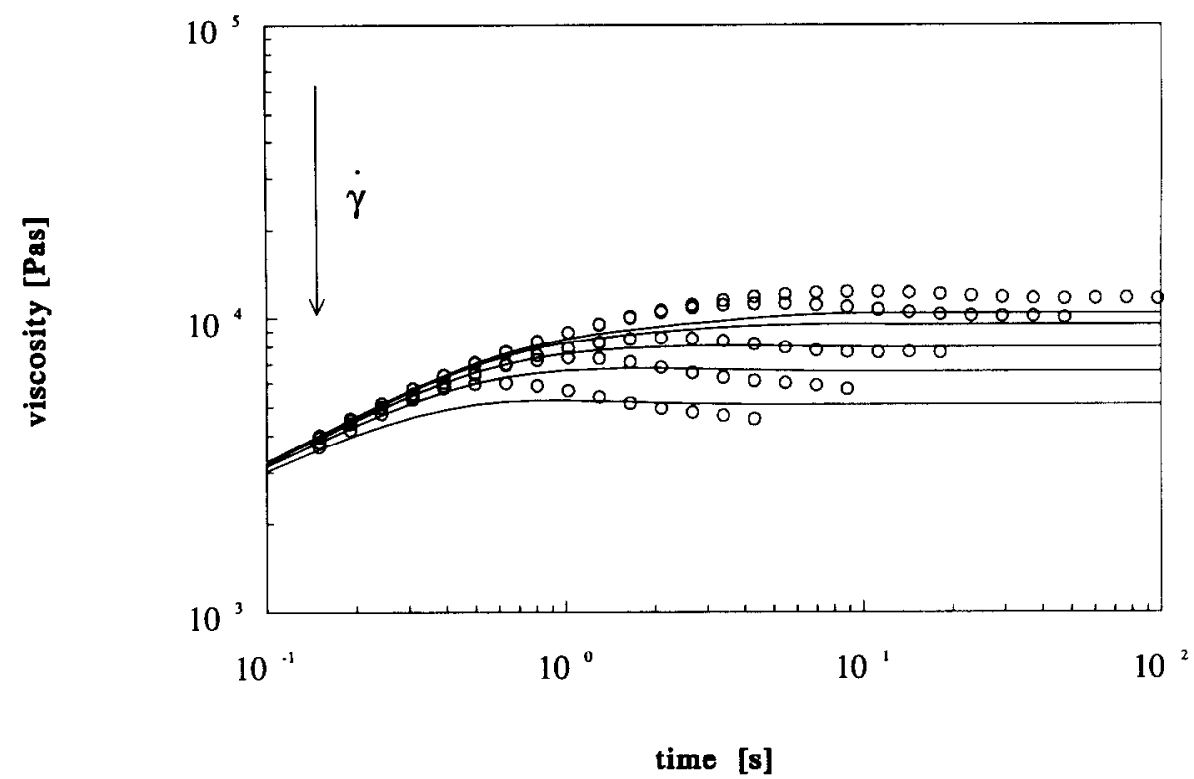

Fig. 6. Transient viscosity for PS 678E for a start-up flow for constant shear rates: $0.2,0.4,1$, $2,4 \mathrm{~s}^{-1}$ at temperature $T=462 \mathrm{~K}$. ( $\circ$ : measured, - : fit).

numerically. Laun ${ }^{54}$ gave an analytical evaluation of the transient viscosity $\eta$ and primary normal stress coefficient $\Psi$ in case of the Wagner model. These are defined as

$$
\eta=\frac{\tau_{12}}{\dot{\gamma}_{0}}, \quad \Psi=\frac{\sigma_{11}-\sigma_{22}}{\dot{\gamma}_{0}^{2}}
$$

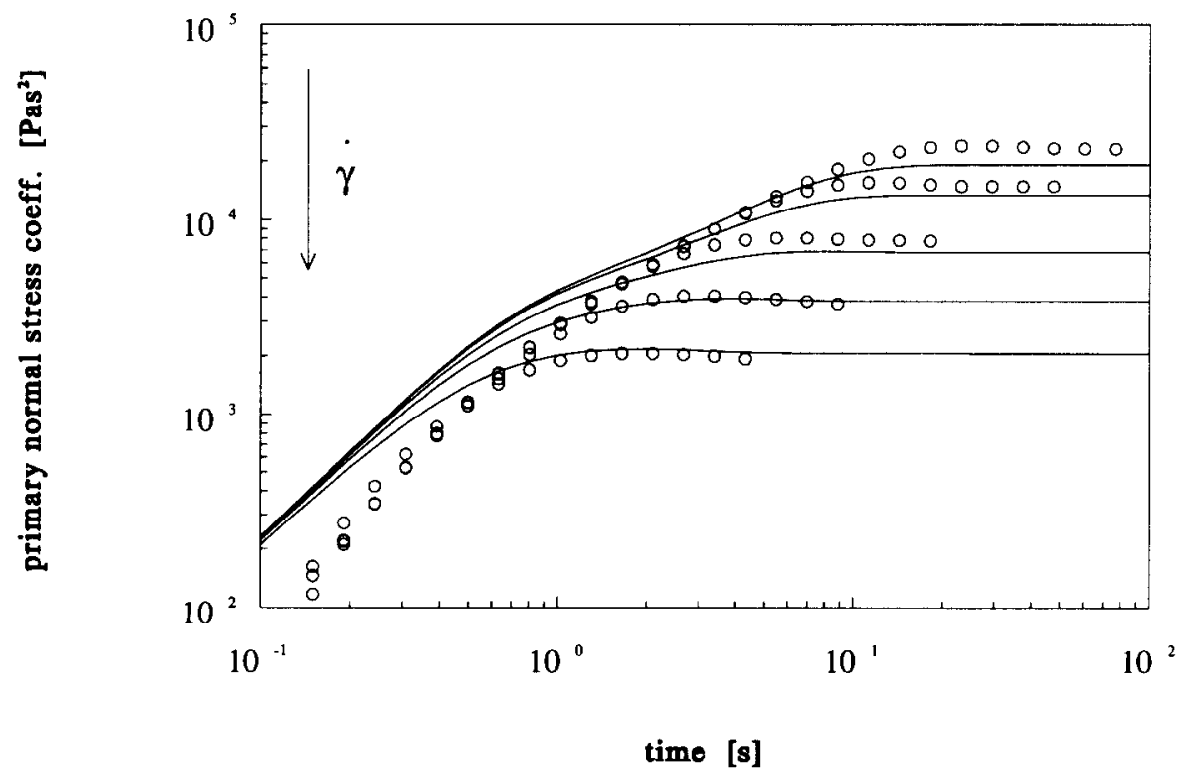

Fig. 7. Transient primary normal stress coefficient for PS $678 \mathrm{E}$ for a start-up flow for constant shear rates: $0.2,0.4,1,2,4 \mathrm{~s}^{-1}$ at temperature $T=462 \mathrm{~K}$. ( $\mathrm{O}$ : measured, - : fit). 
Table 6. Damping function parameters

\begin{tabular}{lcc}
\hline & PS 678E & PC Makrolon CD 2000 \\
\hline$m$ & 0.96 & 0.52 \\
$n_{1}$ & 0.29 & 0.08 \\
$n_{2}$ & 0.086 & 0.98 \\
\hline
\end{tabular}

The results for $\eta$ and $\Psi$ are shown in Figs 8 and 9 for $\dot{\gamma}_{0}=10^{3}, 10^{2}, 10,1$, and $10^{-1} \mathrm{~s}^{-1}$. The expressions for the steady state viscosity for both models were already given by eqs (25) and (26). The largest deviations between the two models are observed in the high shear rate region for $\Psi$. The calculation of flow-induced stresses in injectionmoulded products is very sensitive to the value of $\Psi$ in this region. In order to compare numerical results from the two models (Section 5.2), the damping function has been fitted on the primary normal stress coefficient data of the Leonov model. The resulting parameters of the damping function for both PS and PC are given in Table 7.

\subsubsection{Steady State Shear Viscosity}

From the parameters in Tables 3-6 the steady state shear viscosity according to the Leonov model (eq. (25)) and the Wagner model (eq. (26)) can be determined. Also the empirical Cox-Merz rule ${ }^{19}$ can be employed. This rule states that the steady state viscosity at a certain shear rate equals the modulus of the complex viscosity at an

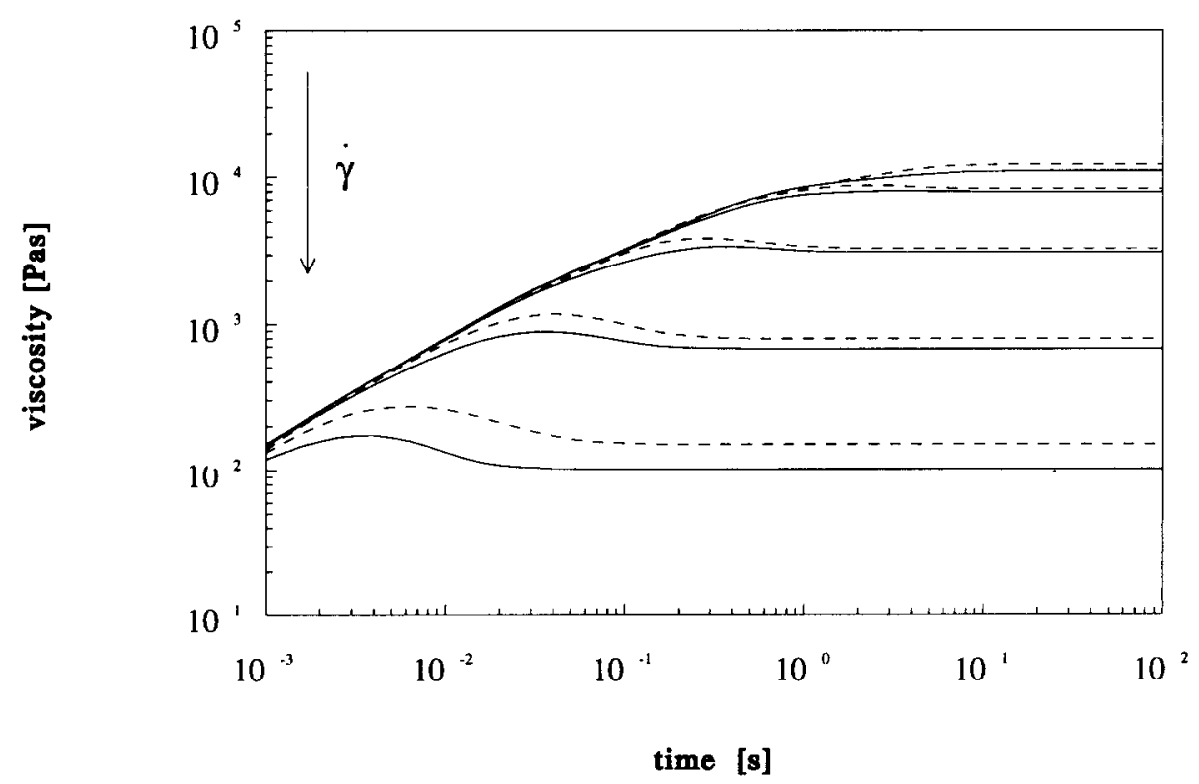

Fig. 8. Transient viscosity for PS 678E according to the Wagner (solid lines) and the Leonov (dashed line) model for a start-up flow for constant shear rates: $10^{-1}, 1,10,10^{2}, 10^{3} \mathrm{~s}^{-1}$ at temperature $T=462 \mathrm{~K}$. 


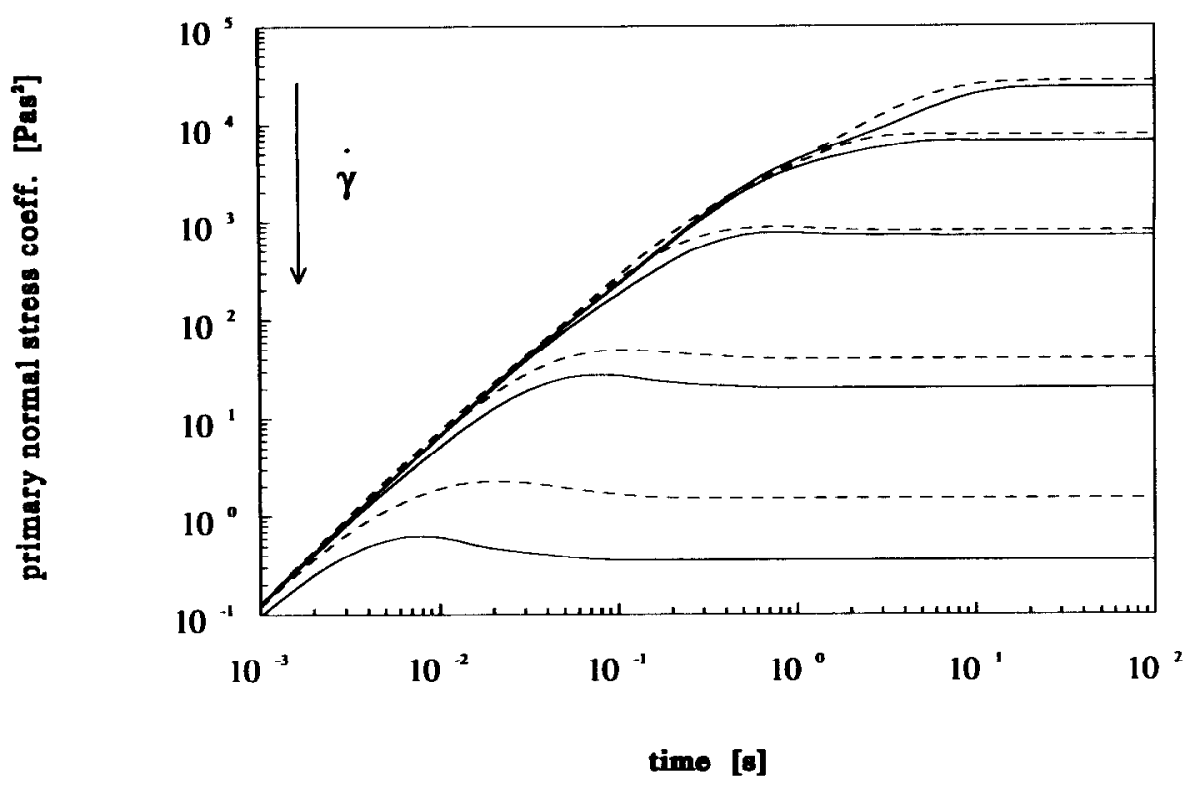

Fig. 9. Transient primary normal stress coefficient for PS 678E. Key as in Fig. 8.

angular frequency equal to the shear rate:

$$
\eta(\dot{\gamma})=\left.\eta_{d}(\omega)\right|_{\omega=\dot{\gamma}}, \quad \eta_{d}=\sqrt{\eta^{\prime 2}+\eta^{\prime 2}}
$$

On a Rheometrics Mechanical Spectrometer RMS-800 at DSM Research, the steady state viscosity of PS is measured at the reference temperature $462 \mathrm{~K}$ for shear rates up to $2 \mathrm{~s}^{-1}$. In the high shear rate region measurements were done on a capillary rheometer at DSM Research. The measured results and the model predictions are displayed in Fig. 10. The experimental results for PS compare well with the model predictions based on the linear viscoelastic measurements.

\subsubsection{Linear Viscoelastic Measurements in the Glass}

Using a rectangular bar geometry, PS $678 \mathrm{E}$ was measured at temperatures between $337 \mathrm{~K}$ and $363 \mathrm{~K}$, in a frequency range $\omega \in\left[2 \cdot 10^{-3}, 500\right] \mathrm{rad} / \mathrm{s}$. Figure 11 shows

Table 7. Damping function parameters, obtained by fitting the start-up shear behaviour according to the Wagner model on the behaviour according to the Leonov model

\begin{tabular}{lcc}
\hline & PS 678E & PC Makrolon CD 2000 \\
\hline$m$ & 0.824 & 0.0595 \\
$n_{1}$ & 0.295 & 0.0386 \\
$n_{2}$ & 0.0575 & 0.2722 \\
\hline
\end{tabular}




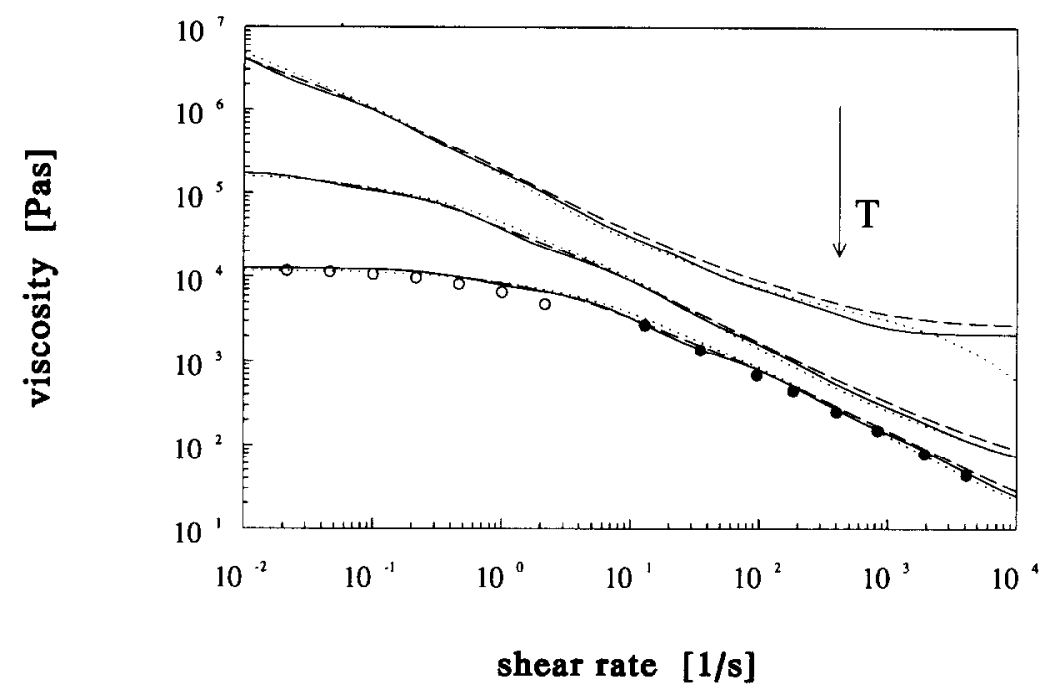

Fig. 10. Steady state shear viscosity as a function of shear rate of PS 678E. Cox-Merz: full line, Leonov: dashed line, Wagner: dotted line, at $T=402,432$, and $462 \mathrm{~K}$; RMS experiments: $O$, capillary rheometer: $\bullet$, at $T=462 \mathrm{~K}$.

the measured loss angle as a function of frequency for all temperatures, and the corresponding mastercurve at $T_{0}=363 \mathrm{~K}$ obtained after horizontal shifts of the individual curves. In Fig. 12, the measured $G_{d}$ is shown, along with its mastercurve. The additional vertical shift is negligible. The horizontal shift factors are displayed in

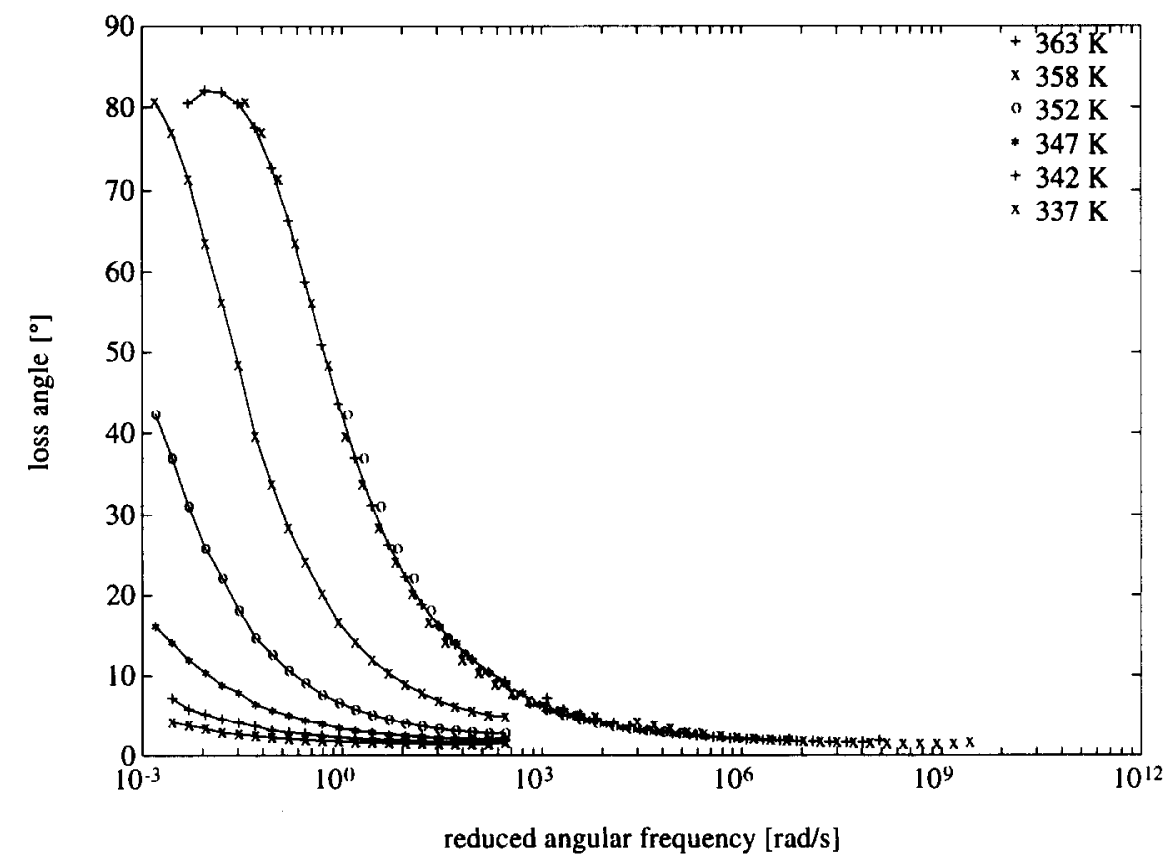

Fig. 11. Loss angle against angular frequency at various temperatures for PS 678E. The reference temperature of the master curve is $363 \mathrm{~K}$. 


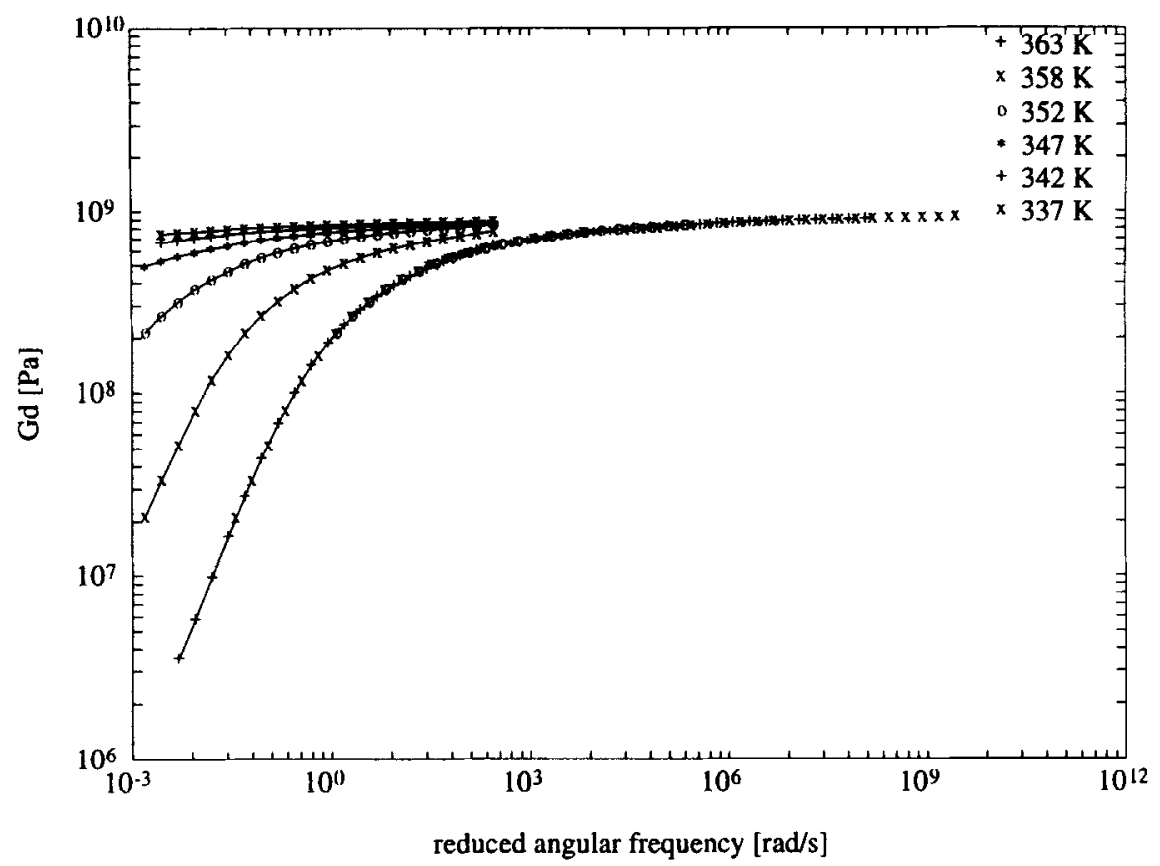

Fig. 12. Dynamic modulus against angular frequency at various temperatures for PS 678E. The reference temperature of the master curve is $363 \mathrm{~K}$.

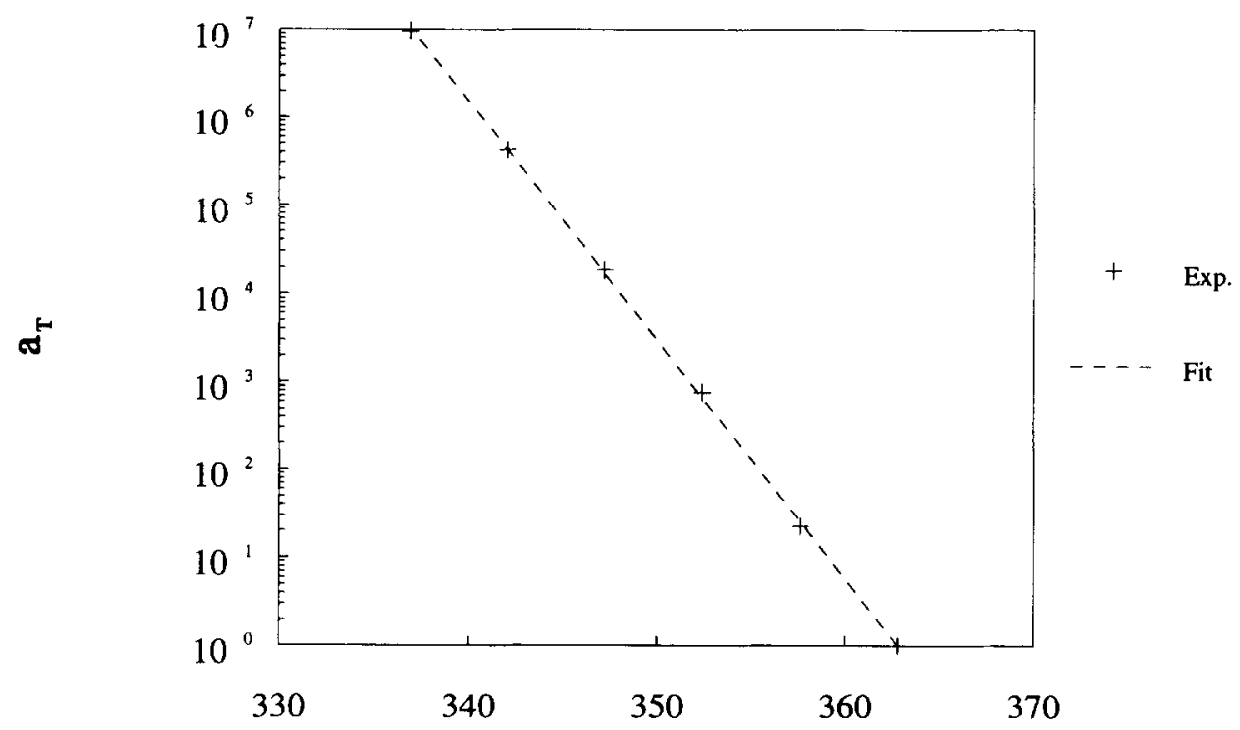

Temperature [K]

Fig. 13. Horizontal shift $a_{T}$ against temperature for PS 678E. (+: experimental, - : fit). 
Table 8. Linear viscoelastic parameters in the glassy state

\begin{tabular}{clccc}
\hline Mode no. & \multicolumn{3}{c}{ PS 678E } & \multicolumn{2}{c}{ PC Makrolon CD 2000 } \\
\hline$i$ & $\theta_{i} s$ & $G_{i} P a$ & $P_{i}$ & $s$ \\
1 & 5.0 & $9.14 \cdot 10^{7}$ & 6.32 & $G_{i} \quad P a$ \\
2 & $3.0 \cdot 10^{-1}$ & $3.43 \cdot 10^{8}$ & $3.53 \cdot 10^{-1}$ & $1.61 \cdot 10^{8}$ \\
3 & $6.66 \cdot 10^{-3}$ & $2.22 \cdot 10^{8}$ & $1.97 \cdot 10^{-2}$ & $1.19 \cdot 10^{8}$ \\
4 & $1.18 \cdot 10^{-4}$ & $1.20 \cdot 10^{8}$ & $1.1 \cdot 10^{-3}$ & $4.83 \cdot 10^{7}$ \\
5 & $2.5 \cdot 10^{-6}$ & $5.96 \cdot 10^{7}$ & $6.13 \cdot 10^{-5}$ & $2.0 \cdot 10^{7}$ \\
6 & $4.77 \cdot 10^{-8}$ & $6.92 \cdot 10^{7}$ & $3.42 \cdot 10^{-6}$ & $1.25 \cdot 10^{7}$ \\
7 & & & $1.91 \cdot 10^{-7}$ & $1.65 \cdot 10^{7}$ \\
\hline
\end{tabular}

Fig. 13. The horizontal shift $a_{T}$ can be fitted by the following expression

$$
a_{T}=e^{-c_{3}\left(T-T_{0}\right)}
$$

The constant $c_{3}$ for PS and $\mathrm{PC}$ is 0.6242 and $0.6015 \mathrm{~K}^{-1}$ respectively. The relaxation function in eq. (33) is fitted on these measurements, yielding six modes for PS and seven modes for PC. The parameters are listed in Table 8.

\section{3. $\mathrm{p}$ T-Data and Thermal Properties}

\subsection{1. $\mathrm{p} \nu \mathrm{T}-$ Data}

The parameters in the Tait equation (37) for both PS 678E and OC Makrolon CD 2000 are listed in Table 9. The fits are plotted in Figs 14 and 15. The data for PS were taken from Flaman, ${ }^{26}$ Chapter 4, and those for PC from Zoller. ${ }^{113}$ It should be noted that the PC data from Zoller were measured on a different type of PC: Lexan 101 from GE.

\subsubsection{Thermal Conductivity, $\lambda$}

Literature data were taken for the isotropic thermal conductivity. Although, previous numerical studies (Sitters, ${ }^{87}$ Chapter 6; Flaman, ${ }^{26}$ Chapter 5) revealed that the calculation results are sensitive to the value of the thermal conductivity, its

Table 9. Parameters in the Tait equation

\begin{tabular}{lcccc}
\hline & \multicolumn{2}{c}{ PS 678E } & \multicolumn{2}{c}{ PC Makrolon CD 2000 } \\
\hline & melt & glass & melt & glass \\
$a_{0} 10^{-4} \mathrm{~m}^{3} / \mathrm{kg}$ & 9.758 & 9.758 & 8.68 & 8.68 \\
$a_{1} 10^{-7} \mathrm{~m}^{3} /(\mathrm{kg} \mathrm{K})$ & 5.8 & 2.3 & 5.77 & 2.2 \\
$B_{0} 10^{8} \mathrm{~Pa}$ & 2.4 & 3.5 & 3.161 & 3.954 \\
$B_{1} 10^{-3}{ }^{\circ} \mathrm{C}^{-1}$ & 3.6 & 3.0 & 4.078 & 2.609 \\
$T_{g}(0) \mathrm{K}$ & 373 & & 423 & \\
$s 10^{-7} \mathrm{~K} / \mathrm{Pa}$ & 5.1 & & 5.2 & \\
\hline
\end{tabular}




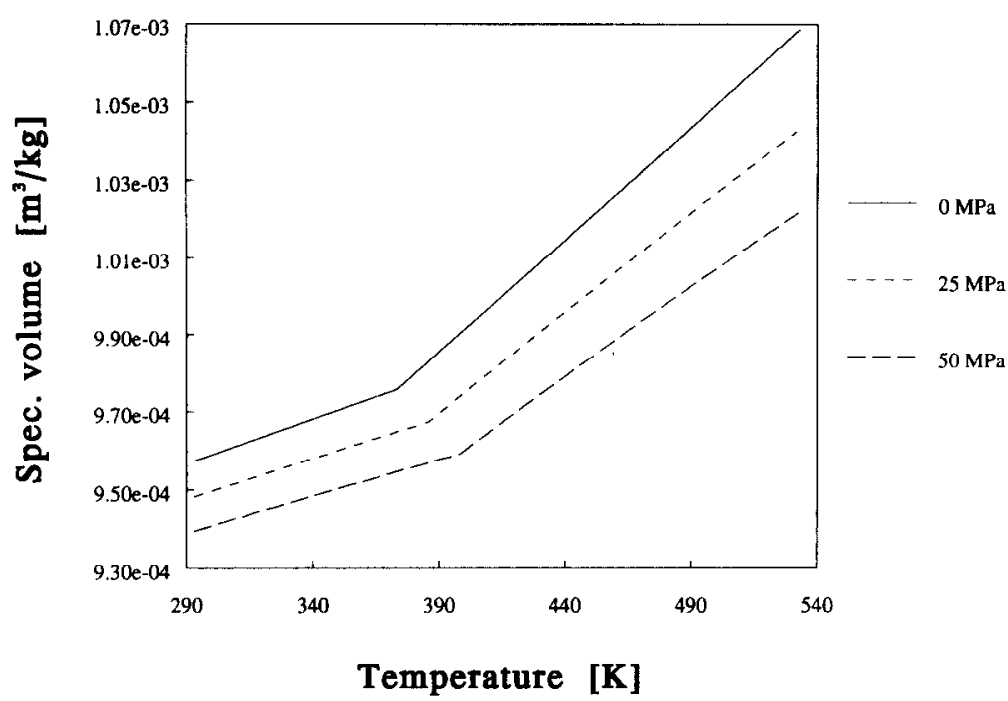

Fig. 14. Specific volume of PS, according to the Tait equation.

variations with respect to the temperature and the pressure are ignored because of lack of experimental data for the materials used in this research. For PS a value of $0.17 \mathrm{~W} /\left(\mathrm{m}^{2} \mathrm{~K}\right.$ ) (see Flaman, ${ }^{26}$ Chapter 4$)$ and, for PC, $0.27 \mathrm{~W} /\left(\mathrm{m}^{2} \mathrm{~K}\right)\left(\right.$ see $\mathrm{VDMA}^{98}$ ) are taken.

The influence of the flow-induced orientation, resulting in an anisotropic conductivity tensor $\lambda$, was investigated by van den Brule, ${ }^{11,12}$ and found to be substantial, mainly in the direction of flow. Since, in injection-moulding, the aspect ratio $h / l$ is small, the main part of the heat conduction is directed perpendicular to the orientation. As a consequence, the approximation of a constant heat conductivity coefficient might not be too bad.

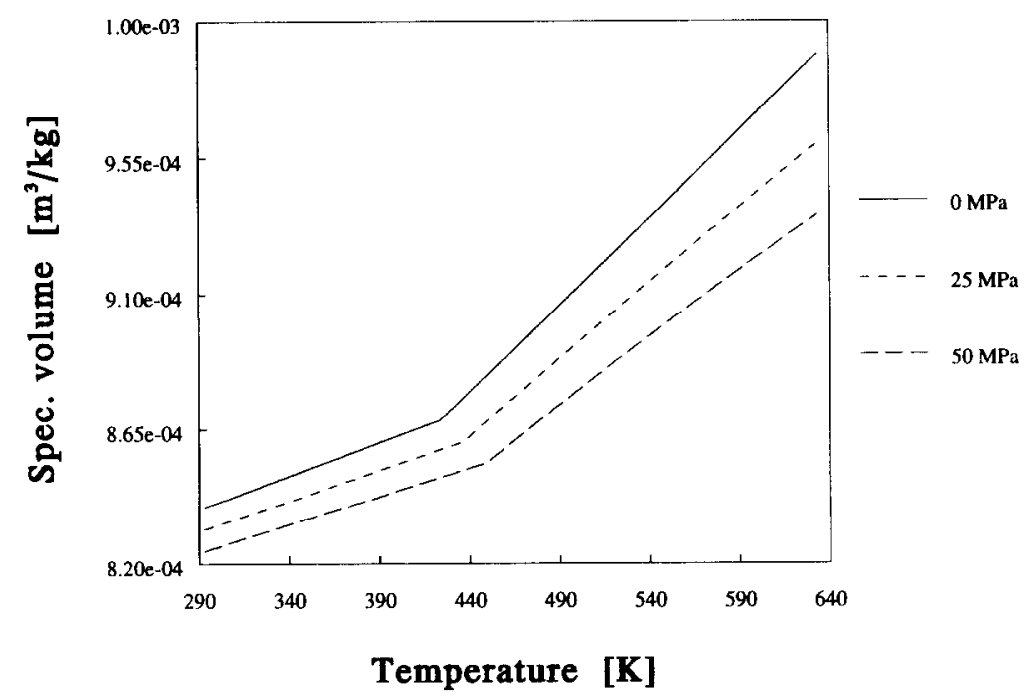

Fig. 15. Specific volume of PC, according to the Tait equation. 


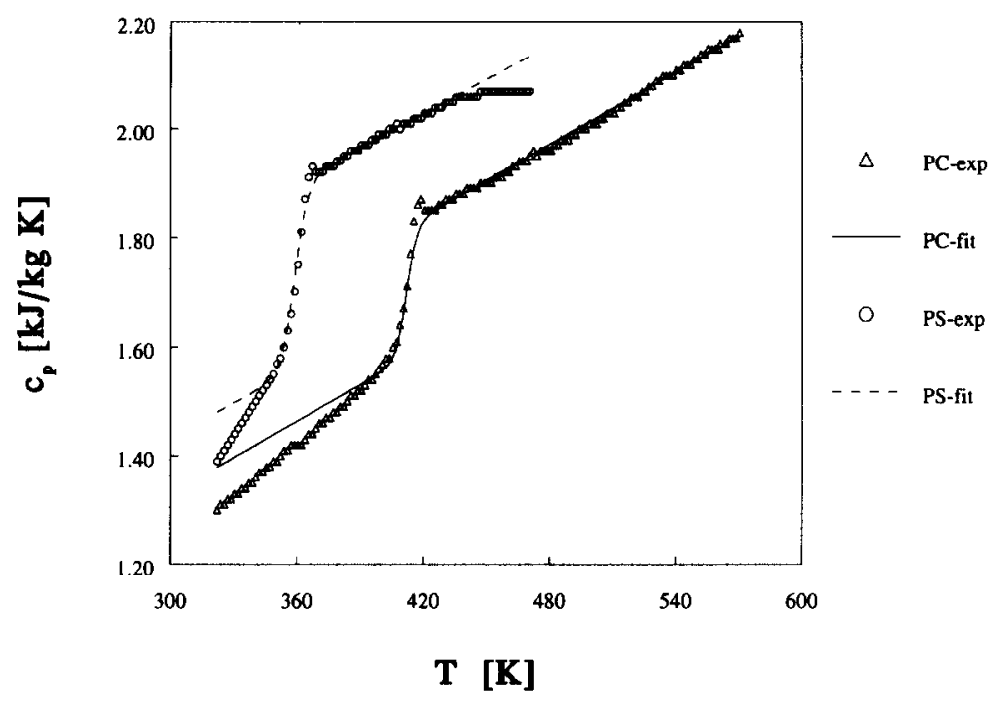

Fig. 16. $c_{p}$ vs temperature for $\mathrm{PC}$ and PS.

\subsubsection{Heat Capacity at Constant Pressure, $\mathrm{c}_{\mathrm{p}}$}

The heat capacity was measured as a function of temperature at atmospheric pressure by means of a Differential Scanning Calorimeter of Perkin-Elmer DSC-7, at DSM Research. The results for both PS 678E and PC Makrolon CD 2000 are provided in Fig. 16. From these measurements also $T_{g}$ at atmospheric pressure can be determined: $T_{g}=359 \mathrm{~K}$ for PS and $T_{g}=412 \mathrm{~K}$ for PC. The pressure dependence of $c_{p}$ was not measured. Chiang et al. ${ }^{14}$ proposed an equation to fit these data:

$$
c_{p}(T)=c_{p 1}+c_{p 2}\left(T-c_{p 5}\right)+c_{p 3} \tan h\left(c_{p 4}\left(T-c_{p 5}\right)\right)
$$

The fit parameters are listed in Table 10; the fits are also displayed in Fig. 16.

\section{NUMERICAL SIMULATIONS}

\subsection{Introduction}

In this chapter, the results of a number of calculations are presented to demonstrate the capabilities of the numerical tools developed, based upon the theory presented.

Table 10. Parameters for $c_{p}$

\begin{tabular}{lcc}
\hline & PS 678E & PC Makrolon CD 2000 \\
\hline$c_{p 1} \mathrm{~J} /(\mathrm{kg} \mathrm{K})$ & 1730 & 1700 \\
$c_{p 2} \mathrm{~J} /\left(\mathrm{kg} \mathrm{K}^{2}\right)$ & 2.1 & 2.2 \\
$c_{p 3} \mathrm{~J} /(\mathbf{k g ~ K})$ & 172 & 120 \\
$c_{p 4} \mathrm{~K}^{-1}$ & 0.155 & 0.2 \\
$c_{p 5} \mathrm{~K}$ & 359 & 412 \\
\hline
\end{tabular}




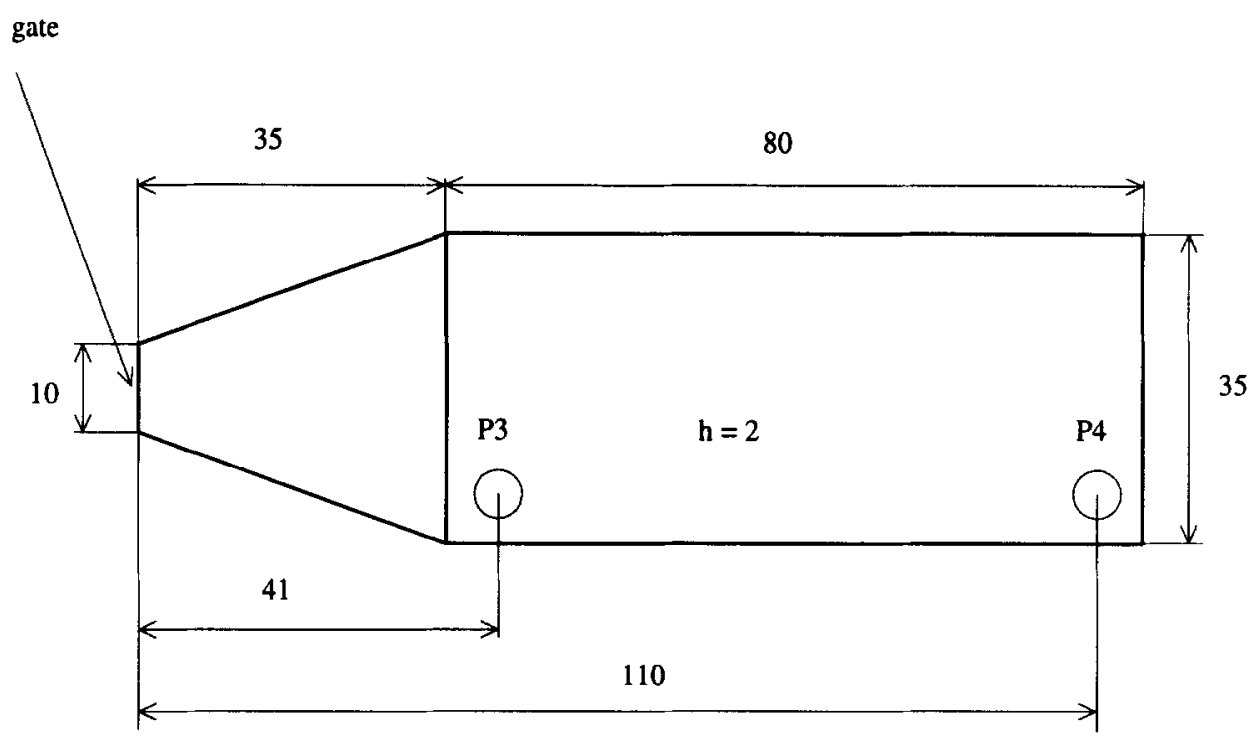

Fig. 17. Mould geometry. P3 and P4 are pressure transducer positions.

Section 5.2 focuses on the computation of flow-induced stresses, results for both the Leonov (1D and 2D) and the Wagner model are shown, while Section 5.3 deals with the calculation of thermally-induced stresses.

\subsection{Flow-Induced Stresses}

\subsubsection{Mould Geometry}

A special test mould, developed at the Philips Plastics and Metalware Factories for research purposes, is considered next. The flat cavity is shown in Fig. 17. This mould was used for experiments focused on the measurement of residual stresses and molecular orientation (Flaman ${ }^{26}$ Schennink ${ }^{83}$ ). For reasons of symmetry only one half of the mould is modelled, see the element mesh in Fig. 18. The height of the rectangular region is $2 \mathrm{~mm}$. In the trapezoidal entrance section, the height ranges from $1.86 \mathrm{~mm}$ on the line of symmetry to $2 \mathrm{~mm}$ on the outer edges in an attempt to create a flat flow front at the start of the rectangular cavity part. Pressure transducers are indicated in Fig. 17 by $\mathrm{P} 3$ and P4.

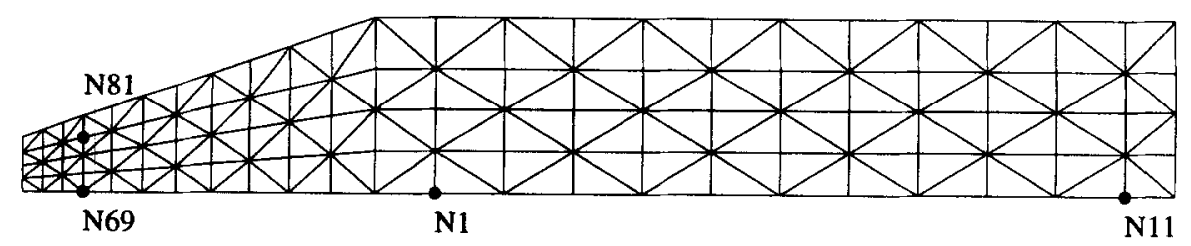

Fig. 18. Element mesh of half the mould. 


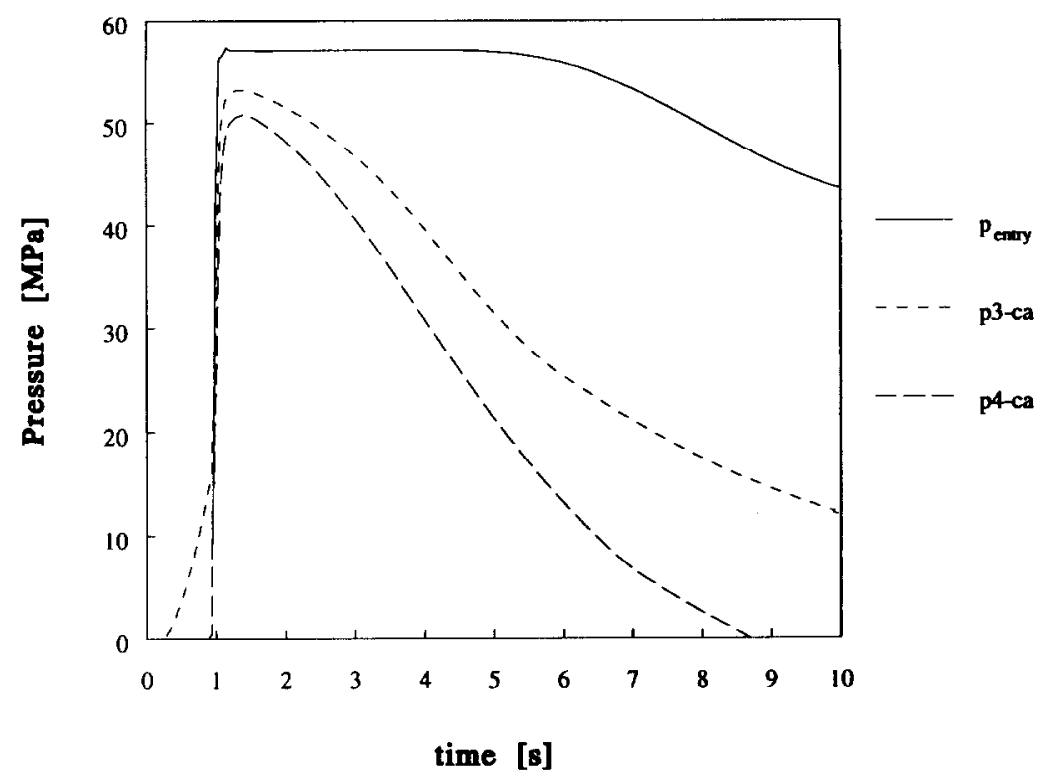

Fig. 19. Pressure histories at the gate, $\mathrm{P} 3$ and $\mathrm{P} 4$.

\subsubsection{Processing Conditions}

The following processing conditions were applied: $T_{i}=473 \mathrm{~K}, T_{w}=323 \mathrm{~K}$, $Q=7.62 \cdot 10^{-6} \mathrm{~m}^{3} \mathrm{~s}$ and for $p_{\text {entry }}$ (see Fig. 19). This case was also investigated by Flaman, ${ }^{26}$ Chapters 6 and 7. Because significant differences in the linear viscoelastic measurements on PS 678E were found, large deviations in the predicted flow-induced stresses are expected.

\subsubsection{Results}

For this case, five calculations are presented. In the first two simulations, the flowinduced stresses are calculated, employing the Leonov model, 1D and 2D (material data: Tables 3, 4). Next the Wagner model is used (material data: Tables 3, 4, 6). Because large differences exist between the computed results of both constitutive equations, in the fourth simulation, the Wagner model is used with the damping function fitted on the rheological behaviour as described by the Leonov model (material data: Tables 3, 4, 7). In the fifth simulation, material data are taken from Flaman, ${ }^{26}$ Chapter 4 , and the results of the computations using the Leonov model, are compared with Flaman's computated and experimental data.

The results for the 1D Leonov analysis are discussed first. Figure 19 shows the pressure histories at the gate (boundary condition) and at the pressure transducer positions P3 and P4. In Fig. 20 the evolution of the first normal stress difference $N_{1}$ is plotted as a function of the dimensionless thickness coordinate, at node N69; see Fig. 18. $N_{1}$ is shown at three times: $t=0.95 \mathrm{~s}$ (end of the filling stage), $t=1.05 \mathrm{~s}$ (end of the packing stage), and at $t=10 \mathrm{~s}$ when the temperature throughout the product has dropped below $T_{g}$, and consequently all flow-induced stresses are frozen-in. At the end 


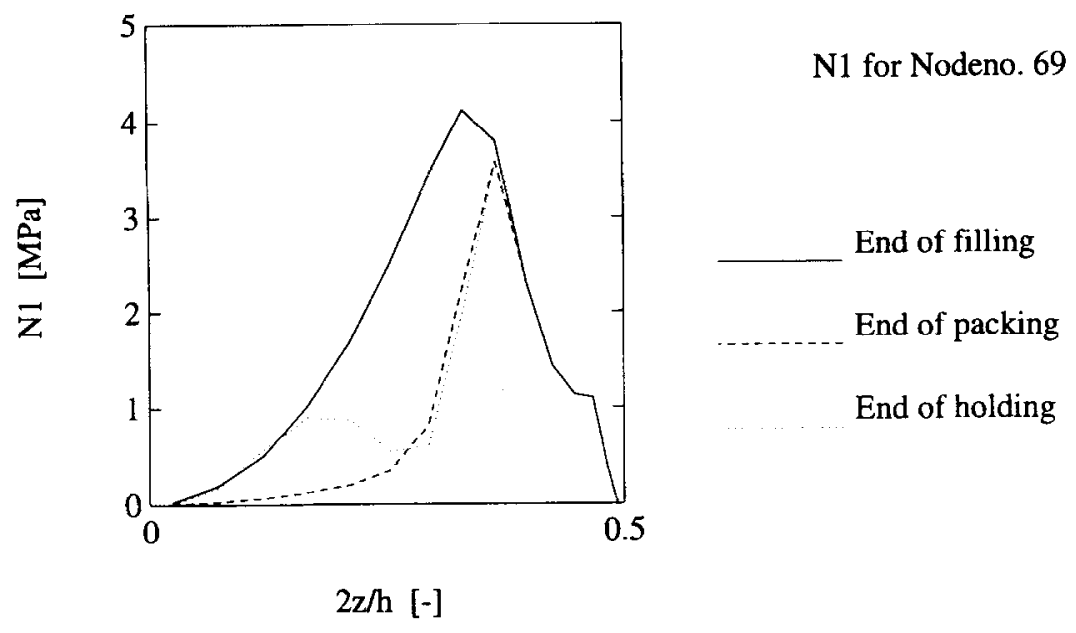

Fig. 20. Evolution of the first normal stress difference as a function of the dimensionless thickness coordinate for the ID calculation with the Leonov model.

of the filling stage, $N_{1}$ is zero in the cavity midplane, rising to a maximum value at a position where the shear rate has a maximum, and decreasing to zero at the surface. Only the stresses near the surface are directly frozen-in, as the solidified layer is growing inwards. At the end of the packing stage, nearly all the stresses in the center are relaxed, because the temperature is still high and the flow rate has dropped dramatically at the completion of the filling stage. During post-filling the shear rate has much lower values compared to those during filling. However, at the same time, the temperature is decreasing, causing a substantial increase of the values of the relaxation times and viscosities. Consequently, flow-induced stresses develop again, now near the centre of the product. The frozen-in first normal stress difference in nodes N69, N1 and N11 is depicted in Fig. 21. The flow-induced stresses decrease in the downstream direction.

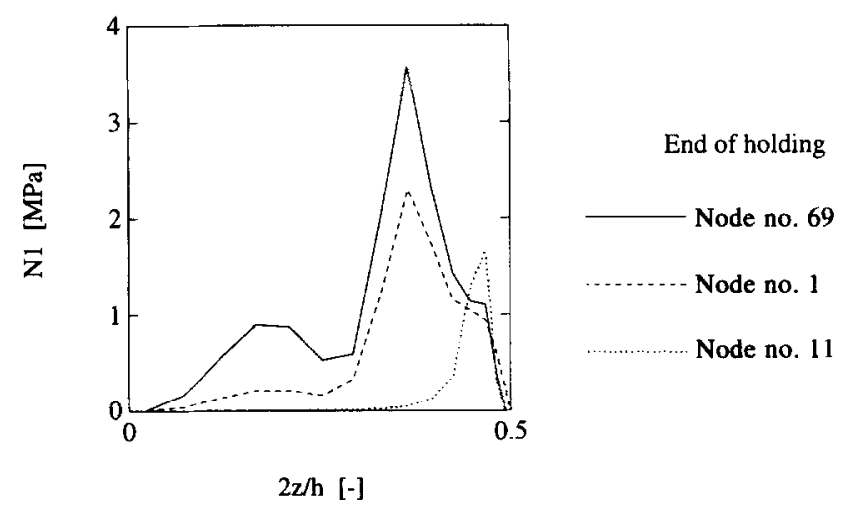

Fig. 21. Frozen-in first normal stress difference as a function of the dimensionless thickness coordinate over the length of the product (nodes N69, N1, and N11), for the 1D calculation with the Leonov model. 

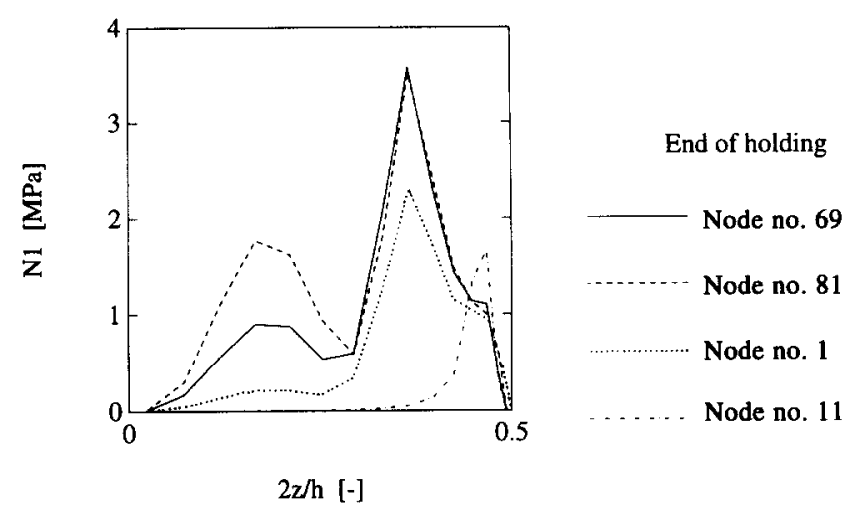

Fig. 22. As Fig. 21, for the 2D calculation with the Leonov model.

The residual $N_{1}$ profilc for the $2 \mathrm{D}$ computation, employing the Leonov model is shown in Fig. 22. The results for the nodes positioned at the line of symmetry: N69, $\mathrm{N} 1$, and N11, coincide with those for the 1D calculation. The results for node N81, differ from those in node $\mathrm{N} 69$ in the centre. Both nodes have the same distance from the line gate. However, the cavity thickness is larger for N81, causing larger flowinduced stresses in the post-filling stage. Figure 23 shows the second normal stress difference $N_{2}=\sigma_{22}-\sigma_{33}$ at the same nodes. The overall values of $N_{2}$ are much lower compared to $N_{1} . N_{2}$ is largest at node N81, where the velocity component in the 2direction is the largest.

Next, the results for the computation employing the Wagner model are discussed. The residual stress $N_{1}$ is shown in Fig. 24. On comparison with the 1D Leonov results in Fig. 21, large differences are observed. In order to check the correctness of the basically different computations, when integral or differential type of constitutive equations are used, an extra analysis is done. In this case, the damping function of the Wagner model is determined by a fit on the rheological behaviour of $N_{1}$, in shear flow, according to the Leonov model. The results for $N_{1}$, plotted in Fig. 25, approximate the results of the 1D Leonov calculation, except for node N69. This clearly

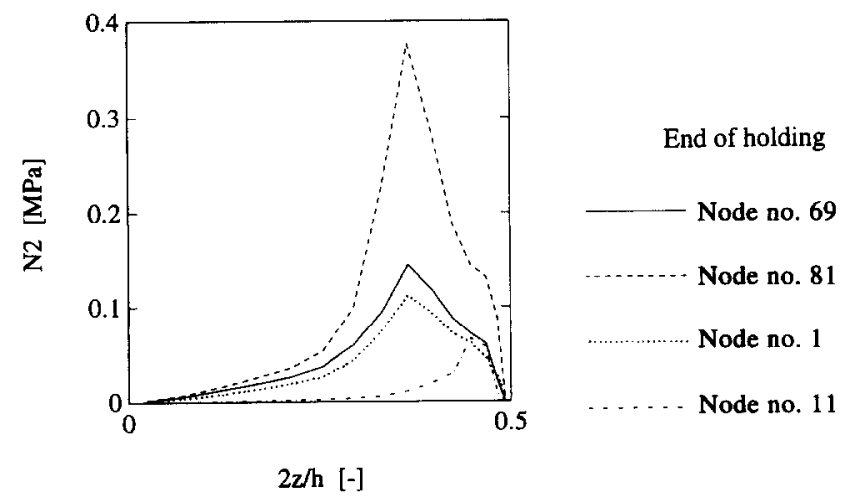

Fig. 23. As Fig. 22, now showing the second normal stress difference $\mathrm{N}_{2}$. 


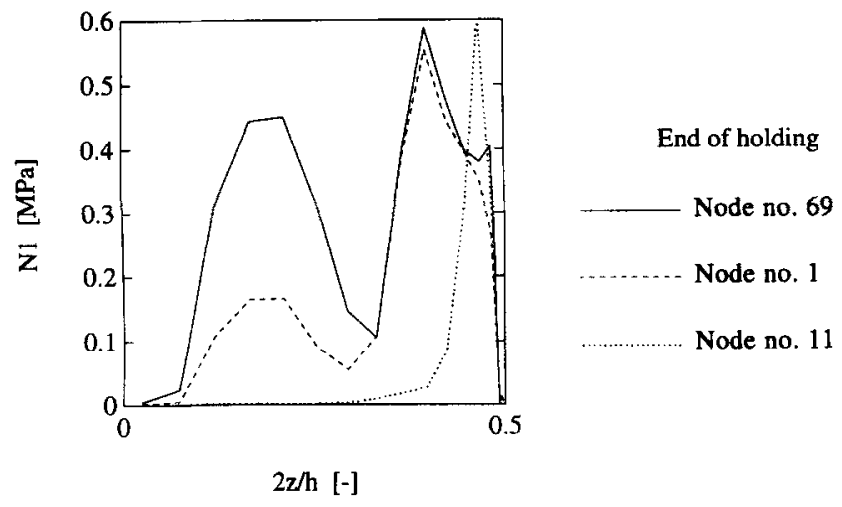

Fig. 24. As Fig. 21, for the 1D calculation with the Wagner model.

shows the sensitivity of the results for the behaviour of $N_{1}$ in a broad range of shear rates.

In the fifth simulation the linear viscoclastic data as determined by Flaman, ${ }^{26}$ Chapter 4 , were used. The results are compared with his fully coupled analysis and with his experiments. Figure 26 shows the birefringence as a function of the thickness coordinate for nodes $\mathrm{N} 1$ and $\mathrm{N} 11$. The birefringence is related to the shear stress and the first normal stress difference via the so-called stress-optical rule:

$$
\Delta n=C \sqrt{N_{1}^{2}+4 \sigma_{12}^{2}}
$$

see e.g. Flaman. ${ }^{26}$ The reasons for the discrepancies between both calculations are differences in $p \nu T$-data used, and the pressure dependence of the viscosity. The decoupled method is known (see Baaijens and Douven ${ }^{3}$ ) to over-predict the frozenin stresses, and consequently the birefringence. As a consequence, not too much value should be attributed to the better comparison of the present calculations with the experimental data.

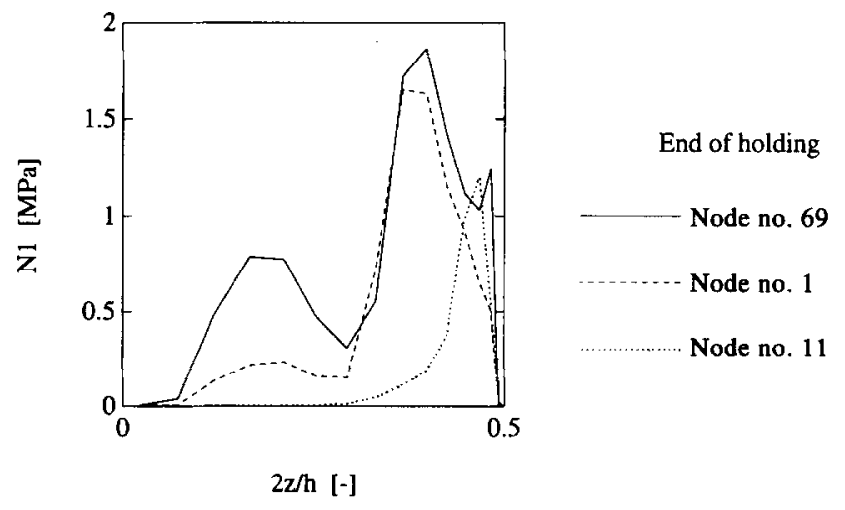

Fig. 25. As Fig. 21, for the 1D calculation with the Wagner model (modified damping function). 


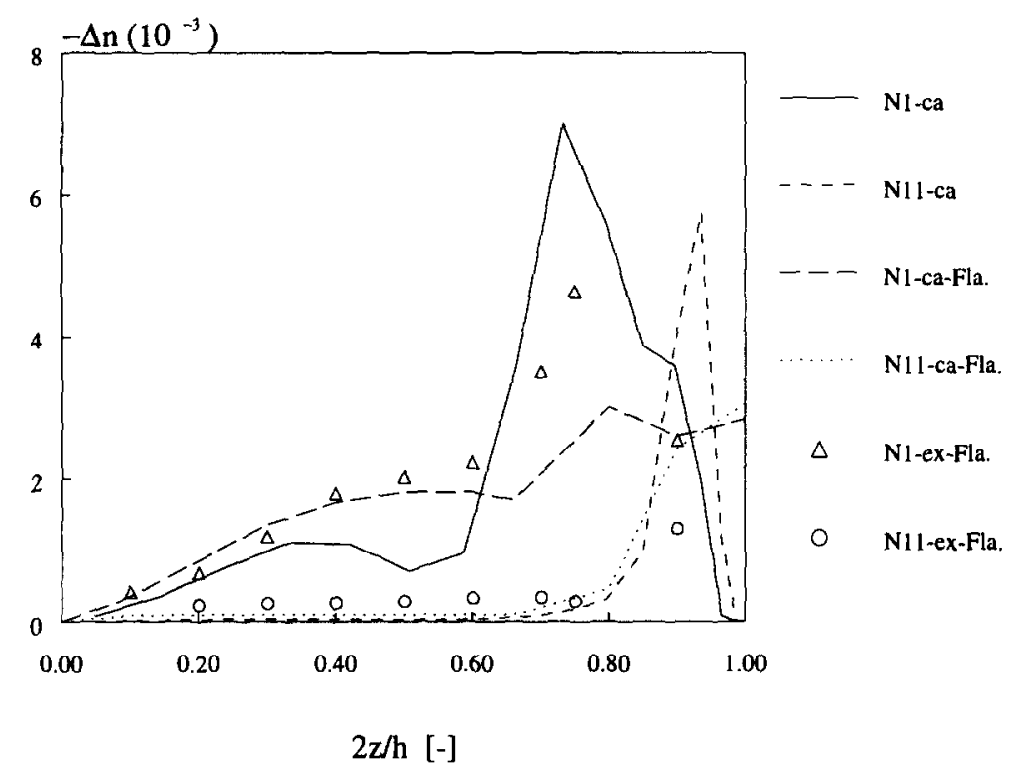

Fig. 26. Frozen-in birefringence as a function of the dimensionless thickness. ca: this work (calculated), ca-Fla: Flaman (calculated), ex-Fla: Flaman (experiments).

\subsection{Thermally-Induced Stresses}

The mould geometry introduced in the previous section is considered again, see Fig. 17. The cases that are investigated are listed in Table 11. Pressure histories at P3 and P4 were recorded by Schennink. ${ }^{83} \mathrm{He}$ also applied the layer removal method (see Treuting and $\operatorname{Read}^{96}$ ) to measure residual stresses, for case no. 1. Case no. 2 is selected to show the effect of asymmetric mould cooling.

\subsubsection{Results}

The finite element mesh, required for the thermal stress problem consists of fournoded quadrilateral shells. These are generated by joining two neighbouring triangles of the mesh generated for the pressure problem, the result is shown in Fig. 27. The elements Q2, Q6, and Q11 are marked in this figure, as they are used for presenting results.

Table 11. Processing conditions

\begin{tabular}{lcc}
\hline \multicolumn{1}{c}{ Case no. } & 1 & 2 \\
\hline material & PS & PS \\
$T_{i} \mathrm{~K}$ & 473 & 473 \\
$T_{w} \mathrm{~K}$ & 328 & $298 / 328$ \\
$\mathrm{Q} 10^{-6} \mathrm{~m}^{3} / \mathrm{s}$ & 7.62 & 7.62 \\
$p_{\text {entry }}$ (see Fig. 29) & 5.13 & 5.13 \\
remark & & asymmetric \\
\hline
\end{tabular}




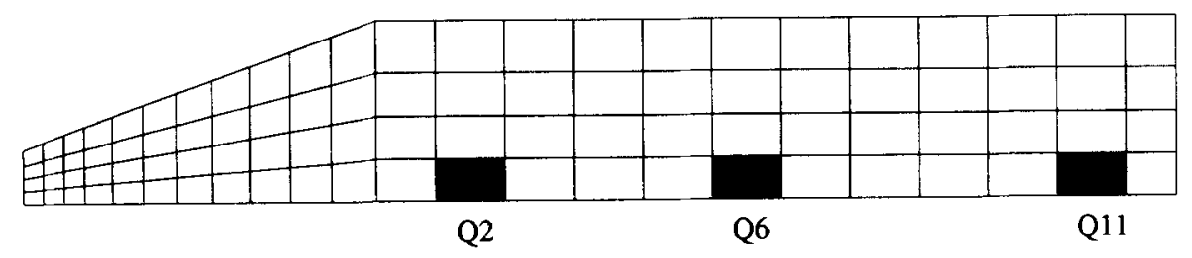

Fig. 27. Quadrilateral element mesh.

\subsubsection{Case No. 1}

In the solidified state, either an elastic or a viscoelastic model can be used to calculate the thermally-induced residual stresses. The parameters for the viscoelastic model are listed in Table 8 , and the elastic shear modulus can be derived from these data: $G_{e l}=0.906 \mathrm{GPa}$.

Also the influence of the type of temperature boundary condition is varied for the calculation employing the viscoelastic model. In one calculation a constant mould wall temperature is assumed, in another one a Biot boundary condition with a (high) heat transfer coefficient of $3000 \mathrm{~J} /\left(\mathrm{m}^{2} \mathrm{sK}\right)$ is used.

Figure 28 shows the calculated filling pattern, and Fig. 29 shows the pressure history at the pressure transducer positions $\mathrm{P} 3$ and $\mathrm{P} 4$, both calculated and measured. In this

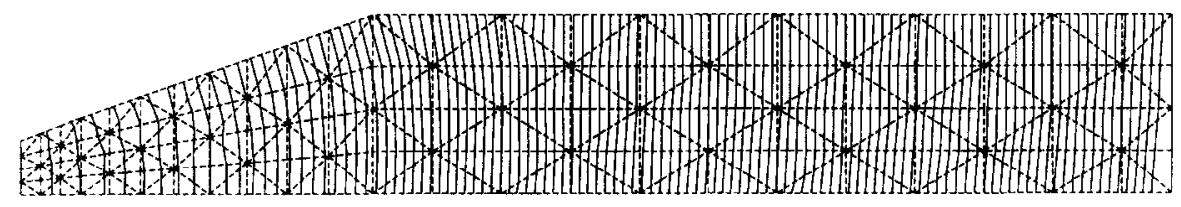

Fig. 28. Filling pattern for case no. 1.

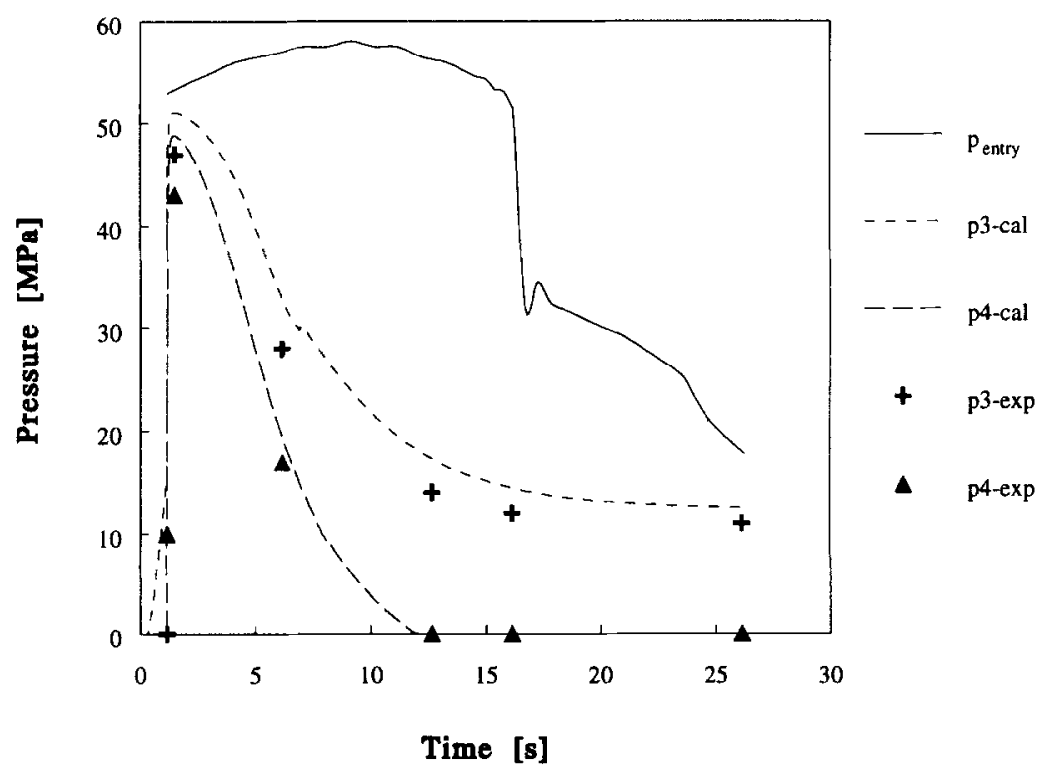

Fig. 29. Pressure histories for case no. 1. 

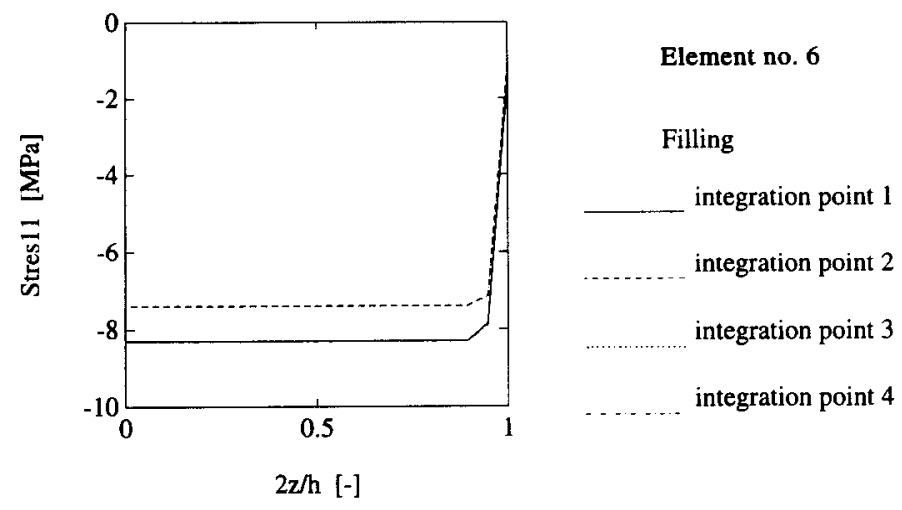

Fig. 30. $\sigma_{11}$ against $2 z / h$ at $t=1.15 \mathrm{~s}$. Element no. Q6, viscoelastic, Biot boundary condition.

last figure also the pressure at the gate, $p_{\text {entry }}$ (boundary condition at the gate during post-filling), is displayed. Compared to the experiments, the calculated results overpredict the pressure decay during post-filling. The calculated thermally-induced stress component $\sigma_{11}$ as a function of the thickness coordinate $z$ in the four integration points of element no. Q6 is shown for the viscoelastic model using a Biot boundary condition. Results are given at the end of filling $(t=1.15 \mathrm{~s})$, end of packing $(t=1.2 \mathrm{~s})$, just before demoulding $(t=25 \mathrm{~s})$, and after demoulding $(t=26 \mathrm{~s})$, in Figs $30,31,32$, and 33 respectively. In the last figure the measured results are given as well. At $t=1.15 \mathrm{~s}$, the bulk is still molten and $\sigma_{11}$ equals minus the melt pressure $p$ (Fig. 30). The surface regions are already solidified, and the prevention of global shrinkage causes relative tensile stresses in these layers. At $t=1.2 \mathrm{~s}$, the packing pressure is applied, and the overall stress level is lowered (Fig. 31). At $t=25 \mathrm{~s}$, just before demoulding, the bulk is solidified, and pressure has decayed towards a non-zero value (Fig. 32). The bulk layers have thus been solidified at decaying pressure and decaying pressure rate. This is reflected by a rise in $\sigma_{11}$ in the bulk region. The residual stress profile after demoulding (see Fig. 33) shows some characteristics: a region of

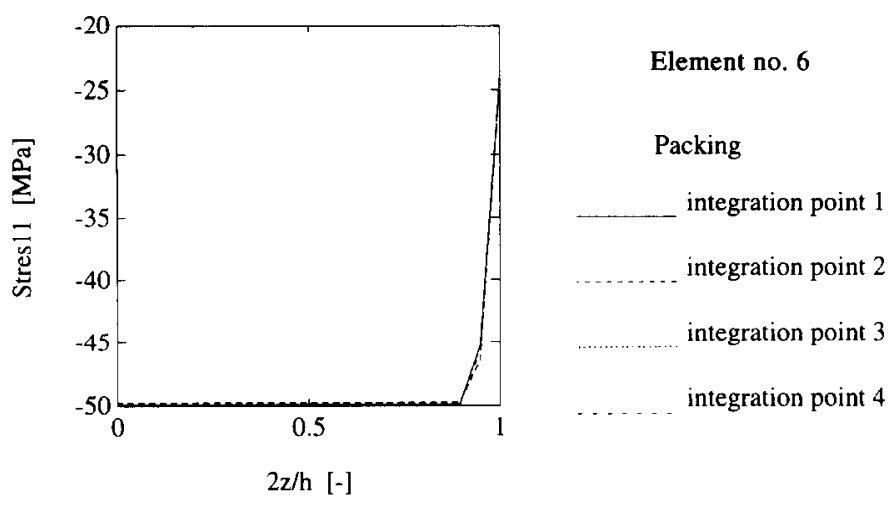

Fig. 31. $\sigma_{11}$ against $2 z / h$ at $t=1.2$ s. Element no. Q6, viscoelastic, Biot boundary condition. 


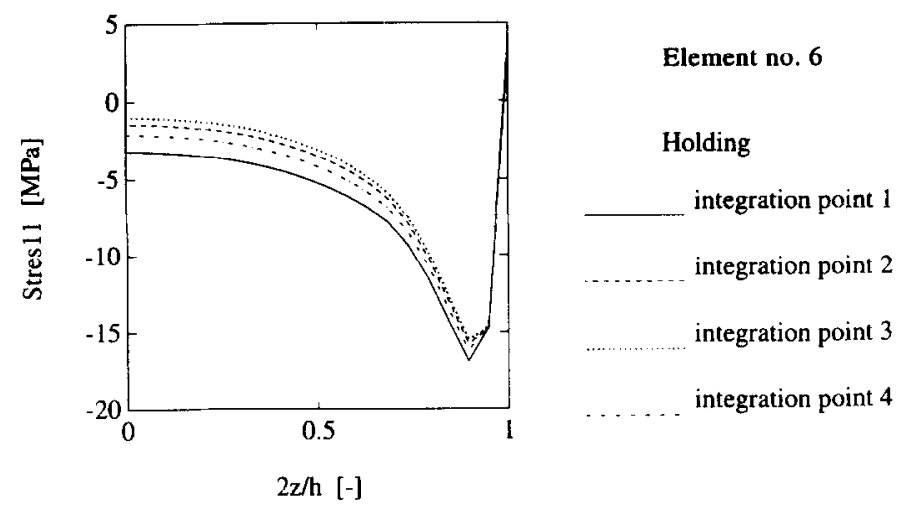

Fig. 32. $\sigma_{11}$ against $2 z / h$ at $t=25 \mathrm{~s}$. Element no. Q6, viscoelastic, Biot boundary condition.

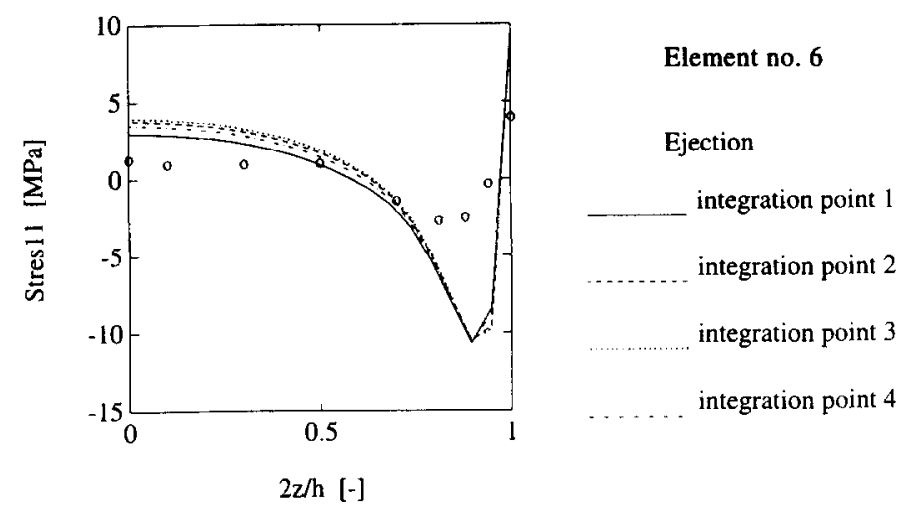

Fig. 33. $\sigma_{11}$ against $2 z / h$ at $t=26 \mathrm{~s}$. Layer removal experiments: $O$. Element no. Q6, viscoelastic, Biot boundary condition.

tensile stresses near the surface, that steeply decays to a compressive minimum that is determined by the level of the packing pressure. The width of this intermediate region is related to the time that the packing pressure can be maintained. In this particular case, this region is narrow. In the bulk a parabolic shaped profile is present, reminiscent of the residual stress profile in a freely quenched sheet. However as seen in Fig. 32 this can also be caused by solidification under decaying pressure and decaying pressure rate. After demoulding no tractions act on the specimen, so the stress profile integrated with respect to $z$ is zero.

In the experimental results, these characteristics are reflected as well, although the absolute values differ considerably from the calculated results. This discrepancy is not understood well. Explanations may be the neglection of the mould elasticity having a marked effect on the pressure history, and the neglect of flow-induced residual stresses. ${ }^{1}$

${ }^{1}$ Zoetelief ${ }^{112}$ also measured $\sigma_{22}$ on injection-moulded strips of ABS and PS - where 1 is the flow direction, and 3 is the thickness direction - by means of the layer removal method, and found differences with $\sigma_{11}$ to be less than $20 \%$. He concluded that since the residual flow-induced stresses were absent in the 2-direction, the influence of these stresses on the measured stress profiles were negligible. 

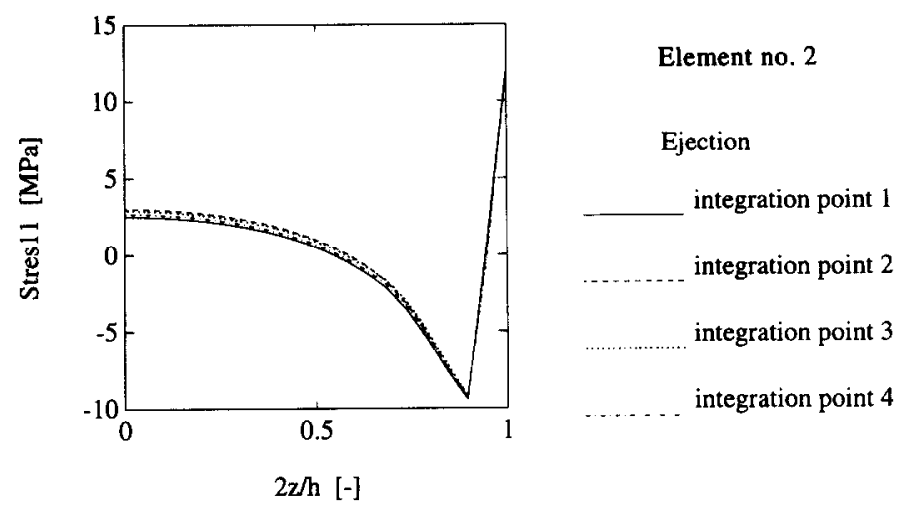

Fig. 34. $\sigma_{11}$ against $2 z / h$ at $t=26 \mathrm{~s}$. Element no. Q2, viscoelastic, Biot boundary condition.

Deviations may also be due to the experimental procedure. A bar is milled from the injection-moulded strip, and subsequently successive layers are milled from this bar. The curvature of the bar as a function of the remaining thickness is used to determine the stress as a function of the thickness coordinate. The curvature is approximated with a polynomial of an arbitrary order. The order of this polynomial, however has a marked effect on the stresses obtained by the layer removal method in the surface region, see Zoetelief ${ }^{112}$ and Schennink. ${ }^{83}$ A higher order polynomial results in higher tensile stresses at the surface, decaying towards higher compressive stresses just below the surface.

The effect of flow length is demonstrated by comparing Fig. 33 with the results for element no. Q2 (upstream) and element no. Q11 (downstream), displayed in Figs 34 and 35 respectively. At the beginning of the flow length the tensile stress at the surface is higher, the tensile stress region is larger, the intermediate minimum is less pronounced, and the bulk region has smaller tension values, compared at the end of the flow length. The differences are attributed to a deviating temperature and pressure history. The broader surface region is explained by the fact that the solidified layer is thicker near the entrance of the cavity, before the packing pressure is applied. At the
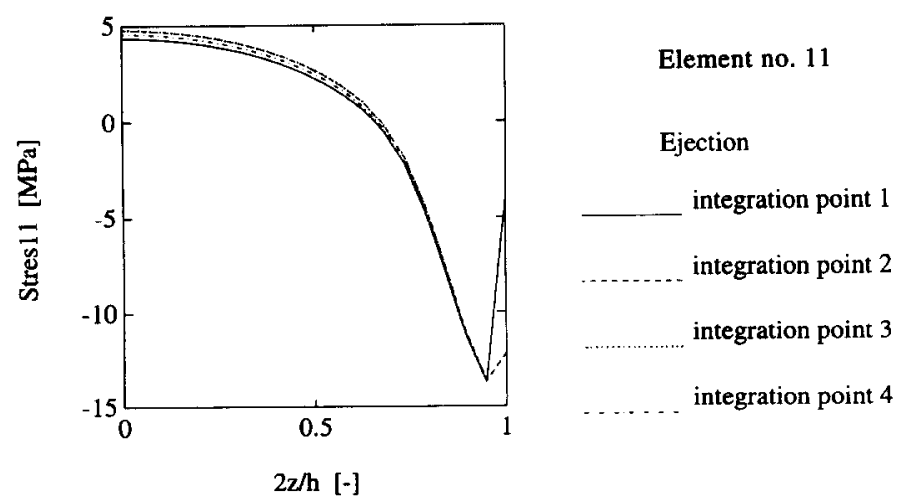

Fig. 35. $\sigma_{11}$ against $2 z / h$ at $t=26 \mathrm{~s}$. Element no. Q11, viscoelastic, Biot boundary condition. 


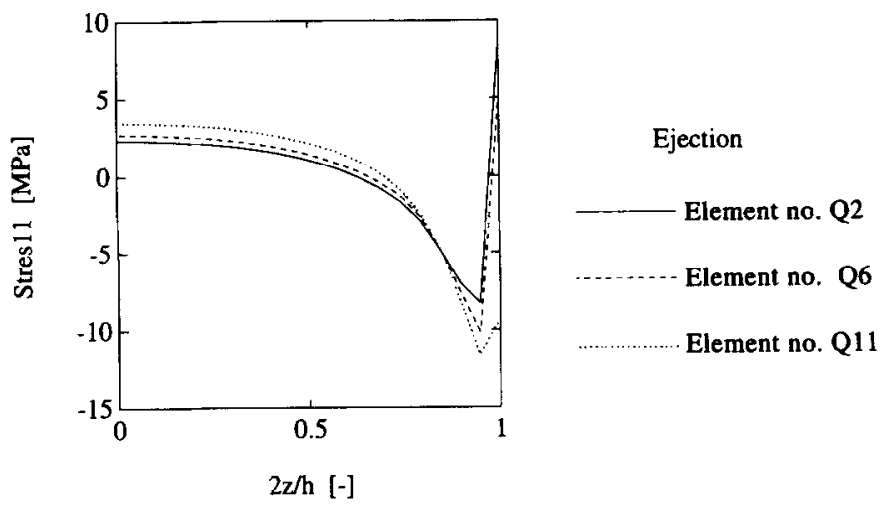

Fig. 36. $\sigma_{11}$ against $2 z / h$ at $t=26 \mathrm{~s}$. Element no. Q2, Q6, and Q11, viscoelastic, Biot boundary condition. Kinematic restriction.

end of the mould, the pressure will decay towards zero, even when the product is still in the mould (see Fig. 29). Thus the material here experiences a free quench situation, and the parabolic shape in the centre is more pronounced, i.e. a larger maximum in the bulk, and a lower minimum in the intermediate zone.

In Fig. 36, the residual stresses are depicted at the elements Q2, Q6, and Q11 for a modified analysis. In this analysis, only the kinematic boundary conditions in the thermal stress problem are changed. The displacements in the 1-direction, are suppressed on the ends of the rectangular cavity part, while the product is still in the mould. The figure shows an overall reduction of the stress levels.

In Fig. 37 the residual stress profiles in element no. Q6 for the elastic shear modulus and a Biot boundary condition are shown. The shear modulus is overpredicted in the elastic case for temperatures close to $T_{g}$. The values at the surface and in the core region differ. Further it is noted that the bulk region has a different shape.

In Fig. 38 the residual stress profiles in element no. Q6 for the shear relaxation modulus and a constant wall temperature are shown. On comparison with the calculation using a Biot boundary condition (Fig. 33), the results display large deviations in the surface region.

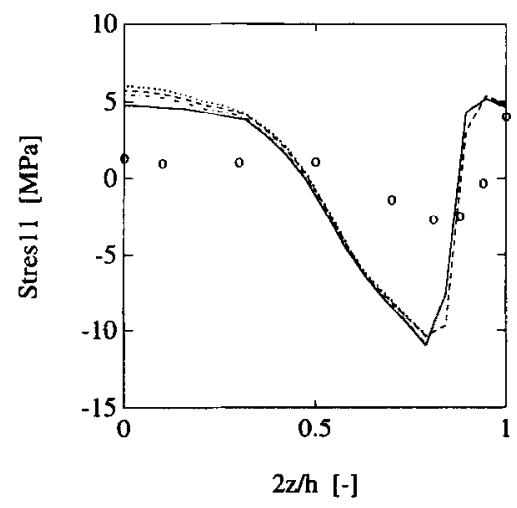

Element no. 6

Ejection

integration point 1

integration point 2

integration point 3

integration point 4

Fig. 37. $\sigma_{11}$ against $2 z / h$ at $t=26 \mathrm{~s}$. Element no. Q6, elastic, Biot boundary condition. 


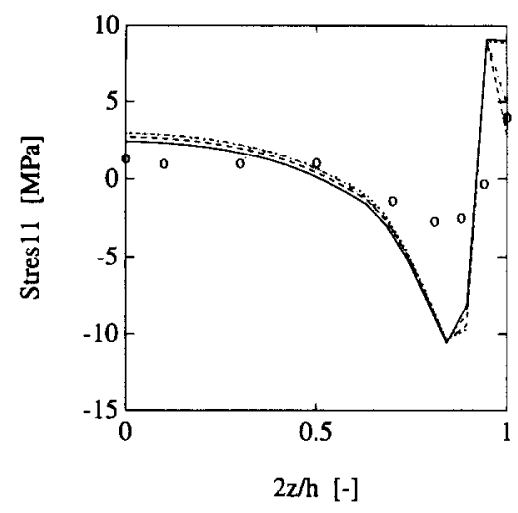

Element no. 6

Ejection

integration point 1

integration point 2

integration point 3

integration point 4

Fig. 38. $\sigma_{11}$ against $2 z / h$ at $t=26 \mathrm{~s}$. Element no. Q6, viscoelastic, constant wall temperature.

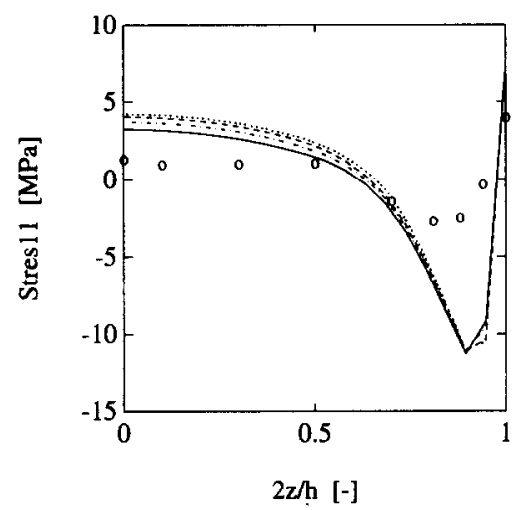

Element no. 6

Ejection

integration point 1

integration point 2

integration point 3

integration point 4

Fig. 39. $\sigma_{11}$ against $2 z / h$ at $t=26 \mathrm{~s}$. Element no. Q6, viscoelastic, Biot boundary condition, and a lower value for $T_{g}: 363 \mathrm{~K}$.

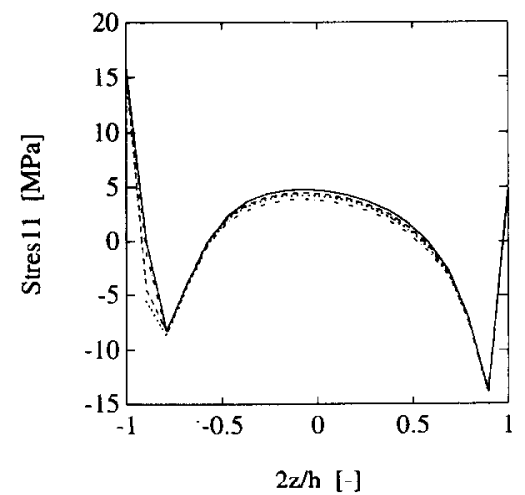

Element no. 6

Ejection

integration point 1

integration point 2

integration point 3

integration point 4

Fig. 40. $\sigma_{11}$ against $2 z / h$ at $t=26 \mathrm{~s}$. Element no. Q6, viscoelastic, Biot boundary condition, and asymmetric cooling. 
In Fig. 39 the residual stress profiles in element no. Q6 for the shear relaxation modulus and a Biot boundary condition are shown again. In this case $T_{g}$ is set to $T_{g}=363 \mathrm{~K}$, to check the influence of the $p \nu T$-data on the results. As can be seen this has only a minor effect.

\subsubsection{Case No. 2}

The results for the asymmetric mould cooling are shown in Fig. 40, where the temperature for $2 z / h=-1$ is $298 \mathrm{~K}$ and for $2 z / h=1$ is $328 \mathrm{~K}$. A very asymmetric stress profile results.

\section{CONCLUSIONS}

In this paper, the injection-moulding process was modelled in order to optimize the process and to predict product properties. Procedures for the calculation of the residual stress state, the orientation, and the dimension and shape of the product were derived. The analysis was restricted to so-called $2 \frac{1}{2} \mathrm{D}$ geometries, i.e. narrow, weakly curved channels. Together, all the constitutive equations used in the modelling must be able to describe the specific material behaviour in the entire range of temperatures, pressures, deformations and deformation rates occurring in the subsequent stages of the process. In the melt the material was assumed to behave as a viscous, generalized Newtonian fluid. However, for flow-induced stresses, the melt behaviour was modelled with a non-linear viscoelastic model. Two constitutive equations were considered: the Leonov model and the Wagner model. The decoupled method, proposed to calculate the flow-induced stresses, seemed to be successful. In order to describe the behaviour of the solidified material, a linearized viscoelastic model was sufficient to calculate the thermally-induced stresses.

To summarize, a number of conclusions are listed, along with some topics recommended for future research.

- Given the state of the art for the analysis of the injection-moulding process, attention should now be focused on a more accurate determination of material properties.

- In this paper it was shown that a quantitatively accurate model for the calculation of flow-induced stresses and molecular orientation can only be attained when the first normal stress difference in shear is measured for high values of the shear rate. This is rather difficult, although, recently, some new experimental procedures were suggested (see Lodge, ${ }^{63}$ and Bulters and Meijer ${ }^{13}$ ).

- For accurate modelling of the pressure decay in the post-filling stage, both the pressure dependence of the viscosity and the (non-equilibrium) $p \nu T$-behaviour should be measured with care.

- Taking into account the total thermo-mechanical behaviour of the mould will improve the model.

- The influence of the extensional flow to the pressure drop and flow-induced 
orientation in centre gated moulds should be investigated. This implies difficult experiments to obtain the elongational viscosity of polymer melts (see Meissner ${ }^{69}$ ).

- In the future, complete 3D modelling of the injection-moulding process should be considered. The spectral element method (Maday and Patera ${ }^{65}$ ) could prove to be a powerful numerical technique in solving the combined pressure and temperature problem. A 3D element with linear or quadratic interpolation of the pressure and a higher order interpolation of the temperature in the thickness dircction should be formulated.

- The results of the flow-induced stress calculation yields the local orientation that can be used for modelling the anisotropic behaviour of the moulded product. Anisotropy of the elastic modulus and heat conductivity can thus be taken into account, e.g. in the calculation of the thermal stresses.

- Visualization of shrinkage and warpage can directly be obtained by plotting the displacements resulting from the solution of the thermal stress problem.

- Presently, the extensional (or fountain) flow in the melt front region is well understood. However, in modelling the injection-moulding process, now only the effect on the convective heat transport is taken into account.

- The residual stress measurements at the surface require extreme accuracy of the experimental procedure. The common methods, the layer removal method and birefringence techniques, might not provide this accuracy (that is important since tensile stresses can be present at the surfaces).

- The stability of the dimensions and shape of the demoulded product should be modelled. The combined effect of relaxation of frozen-in molecular, and frozen-in non-equilibrium density must be considered. A recently developed theory, based on a modified hole theory combines the results of Robertson ${ }^{81}$ and Nies and Stroeks ${ }^{74}\left(\mathrm{Nies}^{73}\right)$. These results could be extremely useful, not only to give a better description of equilibrium $p \nu T$-data, but also for the prediction of nonequilibrium $p \nu T$-data, and the effect of the history of the glass on aging.

- Extensions of the present model are relatively straightforward, and should include the following:

- Modelling of related processes as compression moulding and resin transfer moulding.

- Incorporation of semi-crystalline materials. In the present model, this involves, the introduction of the crystallization kinetics (see Eder et al. ${ }^{24}$ ), the release of heat of crystallization, and the different $p \nu T$-behaviour.

- Modelling the thermoset injection-moulding process, where reaction kinetics, heat of reaction, and the effect of the conversion on the flow behaviour and the modulus should be introduced.

\section{REFER EN CES}

1. F. P. T. Baaijens, Philips Internal Report 311/87, Philips Research Laboratories (1987).

2. F. P. T. Baaijens, Rheoligica Acta 30, 284 (1991). 
3. F. P. T. Baaijens and L. F. A. Douven, Integration of Theory and Applications in Applied Mechanics (J. F. Dijksman and F. T. M. Nieuwstadt, Eds.) pp. 73-90. Kluwer Academic Publishers, Dordrecht, November (1990).

4. G. K. Batchelor, Introduction to Fluid Dynamics, Cambridge University Press, Cambridge (1967).

5. K. J. Bathe and E. N. Dvorkin, Int. J. Num. Meth. Eng. 21, 367-383 (1984).

6. S. Bhattacharji and P. Savic, Proc. 1965 Heat Transf. Fluid Mech. Inst. (A. F. Charwat, Ed.), pp. 248-262. Stanford University Press (1965).

7. R. B. Bird, W. E. Stewart and E. N. Lightfoot, Transport Phenomena, John Wiley \& Sons, New York (1960).

8. A. H. M. Boshouwers and I. J. van der Werf, Inject-3, A Simulation Code for the Filling Stage of the Injection Moulding Process of Thermoplastics. PhD thesis, Eindhoven University of Technology (1988).

9. L. J. Broutman and S. M. Krishnakumar, Pol. Eng. Sci. 16, 74-81 (1976).

10. E. Broyer, Z. Tadmor and C. Gutfinger, Isr. J. Tech. 11, 189-193 (1973).

11. B. H. A. A. van den Brule, Rheologica Acta 28, 257-266 (1989).

12. B. H. A. A. van den Brule and P. J. Slikkerveer, Rheologica Acta 29, 175181 (1990).

13. M. J. H. Bulters and H. E. H. Meijer, J. Non-Newtonian Fluid Mech. 38, 43-80 (1990).

14. H. H. Chiang, C. A. Hieber and K. K. Wang, Pol. Eng. Sci. 31, 116-124 (1991).

15. H. H. Chiang, C. A. Hieber and K. K. Wang, Pol. Eng. Sci. 31, 125-139 (1991).

16. T. S. Chung, Pol. Eng. Sci. 25, 772-777 (1985).

17. T. S. Chung and Y. Ide, J. Appl. Pol. Sci. 28, 2999-3002 (1983).

18. T. S. Chung and M. E. Ryan, Pol. Eng. Sci. 21, 271-275 (1981).

19. W. P. Cox and E. H. Merz, J. Polym. Sci. 28, 619-622 (1958).

20. W. Dietz and J. L. White, Rheologica Acta 17, 676-692 (1978).

21. W. Dietz, J. L. White and E. S. Clark, Pol. Eng. Sci. 18, 273-281 (1978).

22. L. F. A. Douven, Towards the Computation of Properties of Injection Moulded Products: Flow and Thermally Induced Stresses in Amorphous Thermoplastics, $\mathrm{PhD}$ thesis, Eindhoven University of Technology (1991).

23. E. N. Dvorkin and K. J. Bathe, Eng. Comput. 1, 77-88 (1984).

24. G. Eder, H. Janeschitz-Kriegl and S. Liedauer, Prog. Polym. Sci. 15, 629-714.

25. J. D. Ferry, Viscoelastic Properties of Polymers, 3rd edn, John Wiley \& Sons, New York (1980).

26. A. A. M. Flaman, Build-Up and Relaxation of Molecular Orientation in Injection Moulding, $\mathrm{PhD}$ thesis, Eindhoven University of Technology (1990).

27. R. L. Frutiger and T. C. Woo, J. Thermal Stresses 2, 45-60 (1979).

28. R. Gardon, Thermal Stresses in Severe Environments (Hasselman, Ed.) (1980).

29. J. Greener, Pol. Eng. Sci. 26, 886-892 (1986).

30. J. Greener, Pol. Eng. Sci. 26, 534-542 (1986).

31. D. H. Harry and R. G. Parrot, Pol. Eng. Sci. 10, 209-214 (1970).

32. H. S. Hele-Shaw, Proc. R. Soc. 16, 49 (1899).

33. C. A. Hieber, Injection and Compression Molding Fundamentals, chapter 1 (A. I. Isayev, Ed.), Marcel Dekker, Inc. (1987).

34. C. A. Hieber and S. F. Shen, J. Non-Newtonian. Fluid Mech. 7, 1-32 (1980).

35. C. A. Hieber, L. S. Socha, S. F. Shen, K. K. Wang and A. I. Isayev, Pol. Eng. Sci. 23, 20-26 (1983).

36. T. J. R. Hughes and W.k. Liu, Comp. Meth. Appl. Mech. Eng. 26, 331-362 (1981).

37. T. J. R. Hughes, The Finite Element Method, Prentice-Hall International, Inc. (1987).

38. T. J. R. Hughes and T. E. Tezduyar, J. Appl. Mech. 48, 587-596 (1981).

39. A. V. Iacopi and J. R. White, J. Appl. Pol. Sci. 33, 607-623 (1987).

40. A. I. Isayev, Pol. Eng. Sci. 23, 271-284 (1983).

41. A. I. Isayev and T. Hariharan, Pol. Eng. Sci. 25, 271-278 (1985).

42. A. I. Isayev and C. A. Hieber, Rheologica Acta 19, 168-182 (1980).

43. M. St. Jacques, Pol. Eng. Sci. 22, 241-247 (1982).

44. H. Janeschitz-Kriegl, Rheologica Acta 16, 327-339 (1977).

45. H. Janeschitz-Kriegl, Rheologica Acta 18, 693-701 (1979).

46. M. R. Kamal and S. Kenig, Pol. Eng. Sci. 12, 294-301 (1972).

47. M. R. Kamal and S. Kenig, Pol. Eng. Sci. 12, 302-308 (1972).

48. M. R. Kamal, Y. Kuo and P. H. Doan, Pol. Eng. Sci. 15, 863-868 (1975).

49. M. R. Kamal and P. G. Lafleur, Pol. Eng. Sci. 22, 1066-1074 (1982). 
50. M. R. Kamal and P. G. Lafleur, Pol. Eng. Sci. 26, 103-110 (1986).

51. M. R. Kamal and V. Tan, Pol. Eng. Sci. 19, 558-563 (1979).

52. P. G. Lafleur and M. R. Kamal, Pol. Eng. Sci. 26, 92-102 (1986).

53. R. G. Larson, Rheologica Acta 22, 435-448 (1983).

54. H. M. Laun, Rheologica Acta 17, 1-15 (1978).

55. H. Leaderman, Elastic and Creep Properties of Filamentous Materials and Other High Polymers, The Textile Foundation, Washington D. C. (1943).

56. E. H. Lee and T. G. Rogers, J. Appl. Mech. 30, 127-133 (1963).

57. E. H. Lee, T. G. Rogers and T. C. Woo, J. Am. Ceramic Soc. 48, 480-487 (1965).

58. S. Lee, J. de la Vega and D. C. Bogue, J. Appl. Pol. Sci. 31, 2791 2803 (1986).

59. A. I. Leonov, Rheologica Acta 15, 85-98 (1976).

60. A. I. Leonov, J. Non-Newtonian Fluid Mech. 25, 1-59 (1987).

61. A. I. Leonov, E. H. Lipkina, E. D. Paskhin and A. N. Prokunin, Rheologica Acta 15, 411-426 (1976).

62. M. J. Liou and N. P. Suh, Pol. Eng. Sci. 29, 441-447 (1989).

63. A. S. Lodge, J. Rheol. 33, 821 (1989).

64. H. A. Lord and G. Williams, Pol. Eng. Sci. 17, 568-582 (1975).

65. Y. Maday and A. T. Patera, State-of-the-art Surveys on Computational Mechanics, pp. 71-143 (A. Noor, Ed.), ASME (1989).

66. J. F. Mandell, K. L. Smith and D. D. Huang, Pol. Eng. Sci. 21, 1137-1180 (1981).

67. C. E. Maneschy, Y. Miyano, M. Shimbo and T. C. Woo, Exp. Mech. 306-312 (1986).

68. H. Mavridis, A. N. Hrymak and J. Vlachopoulos, Pol. Eng. Sci. 26, 449-454 (1986).

69. J. Meissner, Chimia 38, 35-45 (1984).

70. R. Muki and E. Sternberg, J. Appl. Mech. 28, 193-207 (1961).

71. I. Müller, Thermodynamics, Pitman, London (1985).

72. O. S. Narayanaswamy and R. Gardon, J. Am. Ceramic Soc. 52, 554-558 (1969).

73. E. Nies, Private communication (1991).

74. E. Nies and A. Stroeks, Macromolecules 23, 4088-4092 (1990).

75. S. M. Ohlberg and T. C. Woo, Rheologica Acta 12, 345-348 (1973).

76. A. C. Papanastasiou, L. E. Scriven and C. W. Macosko, J. Rheol. 27, 387 (1983).

77. J. R. A. Pearson, Mechanical Principles of Polymer Processing, Pergamon Press, Oxford (1966).

78. M. Rezayat, SPE ANTEC Tech. Papers 35, 341-343 (1989).

79. S. Richardson, J. Fluid Mech. 56, 609-618 (1972).

80. S. M. Richardson, H. J. Pearson and J. R. A. Pearson, Plast. Rubber Proc. Appl. 5, 55 (1980).

81. R. E. Robertson, R. Simha and J. G. Curro, Macromolecules 17, 911-919 (1984).

82. J. R. Saffell and A. H. Windle, J. Appl. Pol. Sci. 25, 1117-1129 (1980).

83. G. G. J. Schennink, Thermal Stresses in Amorphous Thermoplastics, Master's thesis, Twente University of Technology, in Dutch (1990).

84. H. Schlichting, Boundary Layer Theory, McGraw-Hill, New York (1968).

85. A. Siegmann, A. Buchman and S. Kenig, Pol. Eng. Sci. 22, 560-568 (1982).

86. J. C. Simo, Comp. Meth. Appl. Mech. Eng. 60, 153-173 (1987).

87. C. W. M. Sitters. Numerical Simulation of Injection Moulding, $\mathrm{PhD}$ thesis, Eindhoven University of Technology (1988).

88. M. A. Smits, Philips Internal Report 54/89NE, Philips Centre for Manufacturing Technology (1989).

89. P. So and L. J. Broutman, Pol. Eng. Sci. 16, 785-791 (1976).

90. L. C. E. Struik, Pol. Eng. Sci. 18, 799-811 (1978).

91. S. Subbiah, D. L. Trafford and S. I. Güçeri, Int. J. Heat Mass Transfer 32, 415-433 (1989).

92. Z. Tadmor, J. Appl. Pol. Sci. 18, 1753-1772 (1974).

93. Z. Tadmor, E. Broyer and C. Gutfinger, Pol. Eng. Sci. 14, 660-665 (1974).

94. M. Thompson and J. R. White, Pol. Eng. Sci. 24, 227-241 (1984).

95. G. Titomanlio, V. Ducrato and M. R. Kamal, Int. Pol. Proc. 1, 55-59 (1987).

96. R. G. Treuting and W. T. Read, Jr, J. Appl. Phys. 2, 130-134 (1951).

97. R. K. Upadhyay, A. I. Isayev and S. F. Shen, Rheologica Acta 20, 443-457 (1981).

98. VDMA (Ed.), Kenndaten für die Verarbeitung Thermoplastischer Kunststoffe, vol. 1, Thermodynamik, Carl Hanser Verlag, München (1979).

99. A. H. Wagner, J. S. Yu and D. M. Kalyon, Adv. Pol. Tech. 9, 17-32 (1989). 
100. M. H. Wagner, Rheologica Acta 15, 136-142 (1976).

101. M. H. Wagner, Rheologica Acta 16, 43-50 (1977).

102. M. H. Wagner, Rheologica Acta 18, 33-50 (1979).

103. M. H. Wagner, T. Raible and J. Meissner, Rheologica Acta 18, 427 (1979).

104. J. L. S. Wales, J. Van Leeuwen and R. van der Vijgh, Pol. Eng. Sci. 12, 358-363 (1972).

105. J. L. White and W. Dietz, Pol. Eng. Sci 19, 1081-1091 (1979).

106. S. White, S. Weissman and R. Kambour, J. Appl. Pol. Sci. 27, 2675 (1982).

107. G. Williams and H. A. Lord, Pol. Eng. Sci. 17, 553-568 (1975).

108. M. L. Williams, R. F. Landel and J. D. Ferry, J. Am. Chem. Soc. 77, 3701 (1955).

109. R. Wimberger-Friedl, Orientation, Stress and Density Distributions in Injection-Moulded Amorphous Polymers Determined by Optical Techniques, $\mathrm{PhD}$ thesis, Eindhoven University of Technology (1991).

110. P. C. Wu, C. F. Huang and C. G. Gogos, Pol Eng. Sci. 14, 223-230 (1974).

111. C. J. Wust, Jr and D. C. Bogue, J. Appl. Pol. Sci. 28, 1931-1947 (1983).

112. W. F. Zoetelief, Internal Stresses in Injection-Moulded Products, Master's thesis, Twente University of Technology, in Dutch (1990).

113. P. Zoller, J. Pol. Sci. 20, 1453-1464 (1982). 